\title{
Electrospray ionization mass spectrometry for the analysis of proteins and small molecules
}

\author{
Gregg A. Pratt \\ West Virginia University
}

Follow this and additional works at: https://researchrepository.wvu.edu/etd

\section{Recommended Citation}

Pratt, Gregg A., "Electrospray ionization mass spectrometry for the analysis of proteins and small molecules" (2000). Graduate Theses, Dissertations, and Problem Reports. 1238.

https://researchrepository.wvu.edu/etd/1238

This Dissertation is protected by copyright and/or related rights. It has been brought to you by the The Research Repository @ WVU with permission from the rights-holder(s). You are free to use this Dissertation in any way that is permitted by the copyright and related rights legislation that applies to your use. For other uses you must obtain permission from the rights-holder(s) directly, unless additional rights are indicated by a Creative Commons license in the record and/ or on the work itself. This Dissertation has been accepted for inclusion in WVU Graduate Theses, Dissertations, and Problem Reports collection by an authorized administrator of The Research Repository @ WVU.

For more information, please contact researchrepository@mail.wvu.edu. 


\title{
Electrospray Ionization Mass Spectrometry for the Analysis of Proteins and Small Molecules
}

\author{
Gregg A. Pratt \\ DISSERTATION
Submitted to the Eberly College of Arts and Sciences
at West Virginia University \\ in partial fulfillment of the requirements for the degree of \\ Doctor of Philosophy \\ in \\ Chemistry
}
Fred L. King, Ph.D., Chair
Patrick S. Callery, Ph.D.
Paul W. Jagodzinski, Ph.D.
Ronald B. Smart, Ph.D.
Aaron T. Timperman, Ph.D.
Department of Chemistry
Morgantown, West Virginia
2000

Keywords: electrospray, mass spectrometry, heme proteins, conformations 


\section{ABSTRACT \\ Electrospray Ionization Mass Spectrometry for the Analysis of Proteins and Small Molecules Gregg A. Pratt}

In this document methods for the analysis of proteins and small molecules by Electrospray Ionization Mass Spectrometry (ESI-MS) are discussed. Section I contains an introduction to ESIMS, including a brief history of mass spectrometry, a discussion of the current theories developed to explain the mechanisms at work during the electrospray process, and an explanation of Ion Trap Mass Spectrometry (IT-MS), the type of mass analyzer used for the studies outlined in subsequent chapters. .

Section II is devoted to the analysis of proteins by ESI-MS. Chapter 2 gives an overview of protein structure and reviews previous work in the area of protein structural analysis by ESIMS. Chapter 3 summarizes experimental work done in our laboratory with regard to the influence of instrumental parameters on the Charge State Distribution (CSD) of the heme proteins cytochrome $\mathrm{c}$ and myoglobin, and discusses a method for monitoring conformational changes of proteins by calculating an Average Observed Charge (AOC) for a given spectrum.

Chapter 4 is also devoted to protein conformational analysis by ESI-MS. This work outlines the changes to the CSD and AOC when an organic sheath liquid is infused to the ion source and co-electrosprayed with the aqueous solution containing the proteins. In Chapter 5 Collision Induced Dissociation (CID) is utilized to determine the energy required to liberate the prosthetic heme group from the various charge states of myoglobin, in which the heme is not covalently associated with the polypeptide portion of the molecule, and cytochrome $\mathrm{c}$ in which the heme group is tethered to the polypeptide by two covalent linkages. Heme ion intensity is monitored as a function of applied collision energy, and the results are displayed graphically in dissociation plots. From the plots it can be seen that less energy is required to liberate the heme group from the higher charge states. Additionally, dissociation plots for like charge states of the 
two proteins are directly compared. From this comparison it is observed that significantly more energy is required to dissociate the heme group form cytochrome $\mathrm{c}$ than from myoglobin, consistent with the stronger heme:protein association due to the presence of the covalent bonds in cytochrome $\mathrm{c}$ that are absent in myoglobin.

Chapter 6 details multiple-stage mass analysis $\left(\mathrm{MS}^{\mathrm{n}}\right)$ to determine the fragmentation pathways followed by the heme groups liberated by CID from myoglobin and cytochrome c. A mass difference of $1 \mathrm{AMU}$ is observed for the heme groups from the two proteins, though the heme group is of the same type. The mass difference appears to create a bias toward one of two competing fragmentation pathways that are observed to some degree in the heme groups of both proteins.

Section III outlines work in the analysis of small molecules by ESI-MS. Chapter 7 describes the identification of a urinary metabolite of nicotinylalanine by LC/ESI-MS. The identification is made by comparison of data obtained from LC retention time analysis, and single-stage and multiple-stage mass analysis of the urinary metabolite and the synthetic material. A curriculum vita (CV) for the author follows Section III, and References conclude this document 


\section{Acknowledgements}

I would like to gratefully acknowledge the assistance of my advisor, Dr. Fred King, whose guidance and support throughout the course of my program have been indispensable. His ability to lead, not by force but by kind suggestion, is a model for the role of leadership upon which I now embark. It is my sincere wish that I may live up to the standard. I would also like to thank Dr. Patrick S. Callery, Dr. Paul W. Jagodzinski, Dr. Ronald B. Smart, and Dr. Aaron T. Timperman for serving as committee members, and for their assistance in other matters throughout the years.

Additionally, I would like to thank my parents, Mr. and Mrs. H.R. Pratt, Jr. for their unwavering support during the best, and the worst of times. Their belief in me has known no boundaries, and for the love and support which they have provided I am eternally grateful.

Finally, and most importantly I would like to thank my wife, Amy Pratt, for granting me the opportunity to fulfill a dream. We together have sacrificed much for me, after years in industry and advanced in age to return to school to pursue my doctoral degree. Her undying support and unending belief in me have allowed us both to walk this "road less traveled", that has indeed made all the difference. 


\section{Table of Contents}

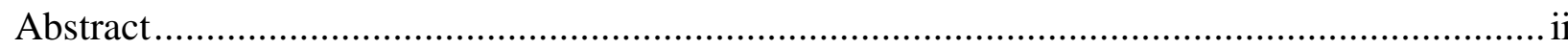

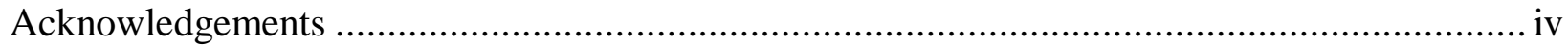

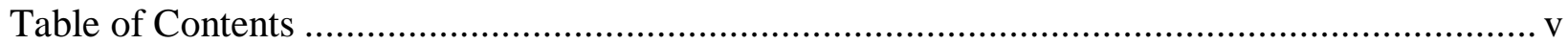

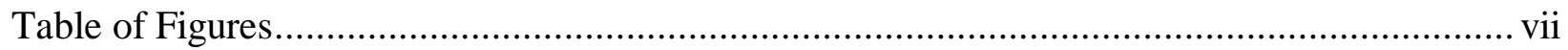

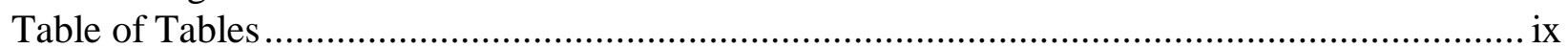

Section 1: Electrospray Ionization Mass Spectrometry .......................................................... 1

Chapter 1: An Introduction to Electrospray Ionization Mass Spectrometry .............................. 2

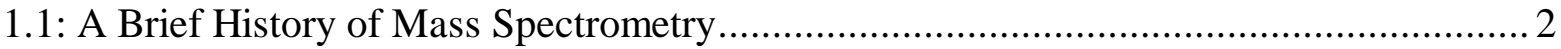

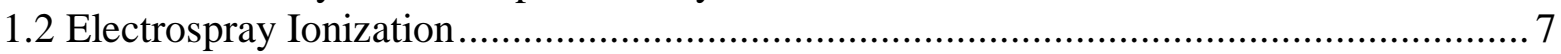

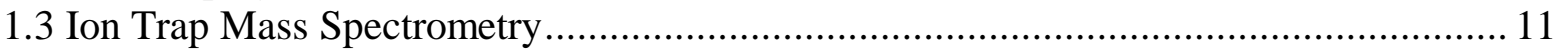

Section 2: Analysis of Proteins by Electrospray Ionization/Ion Trap Mass Spectrometry ........... 17

Chapter 2: Electrospray Ionization Mass Spectrometry for the Analysis of Proteins................ 18

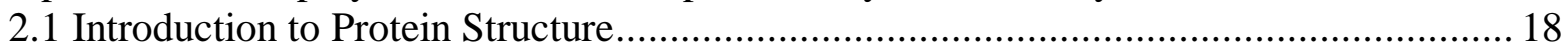

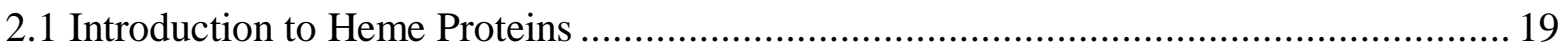

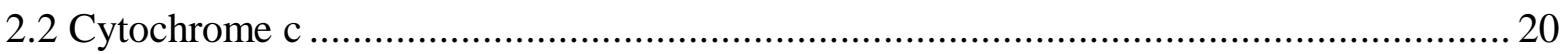

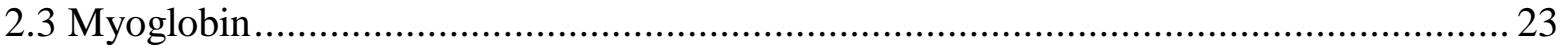

2.4 Electrospray Ionization Mass Spectrometry for Protein Analysis ................................. 27

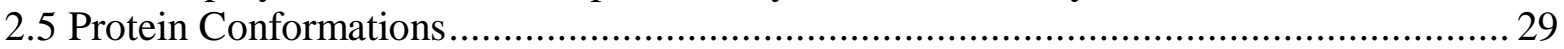

2.6 Protein Conformational Analysis by Electrospray Mass Spectrometry .......................... 30

Chapter 3: Influence of Ion Source Parameters on the Charge-State Distributions of

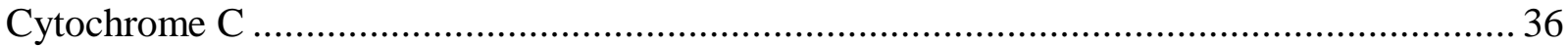

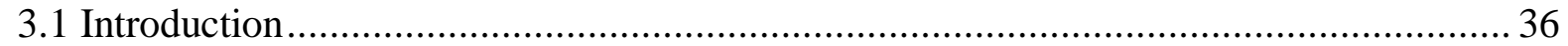

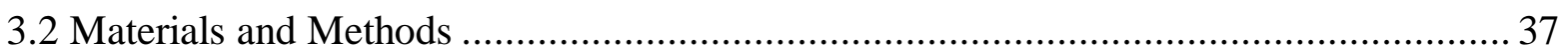

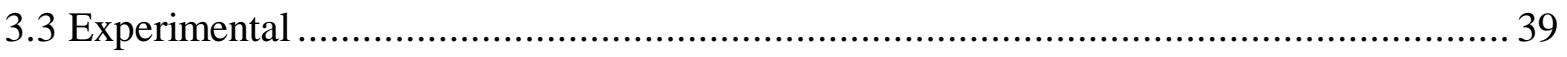

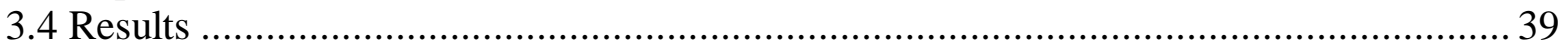

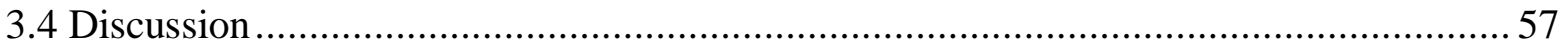

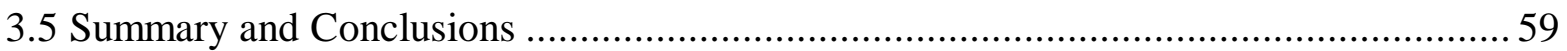

Chapter 4: Influence of Sheath Liquid Composition on the Charge State Distributions of Heme

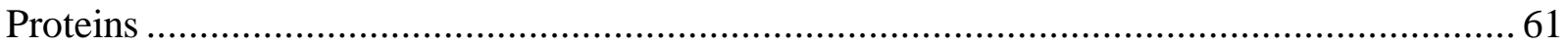

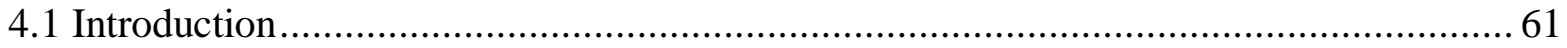

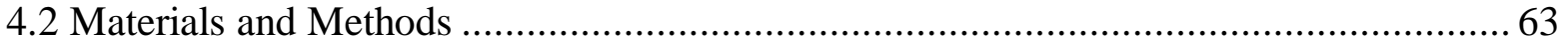

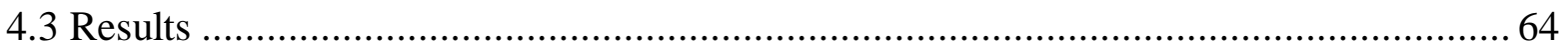

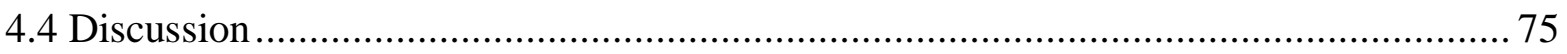

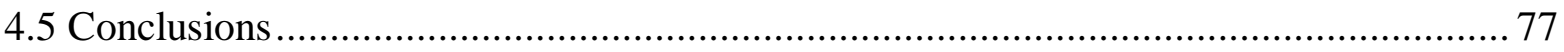

Chapter 5: Energetics of Heme Dissociation from Heme-Containing Proteins: Evidence of the Correlation Between Charge States and Conformational States in the ESI/MS Analysis of

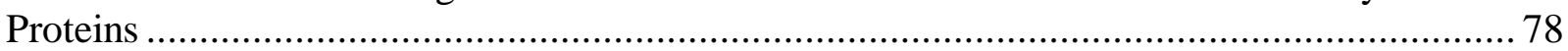

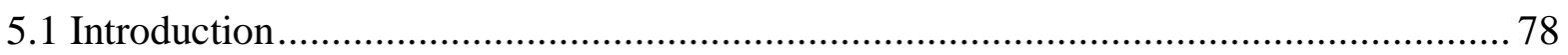

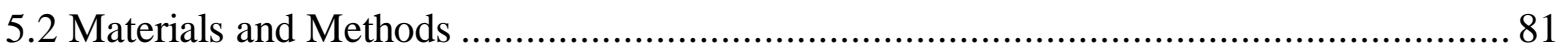

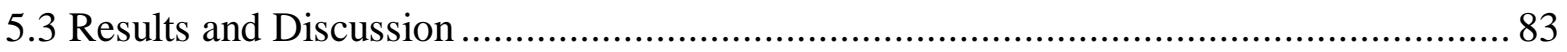

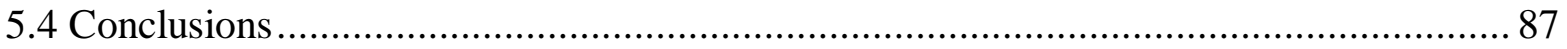


Chapter 6: The Dependence of the Observed Fragmentation Pattern on the Oxidation State of

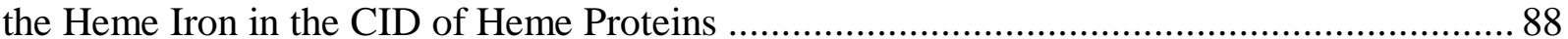

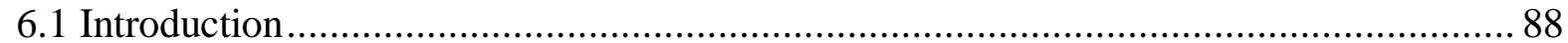

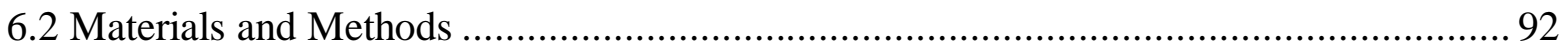

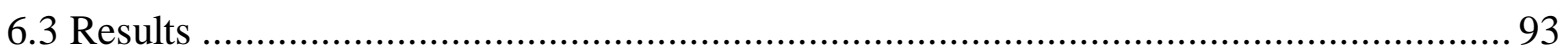

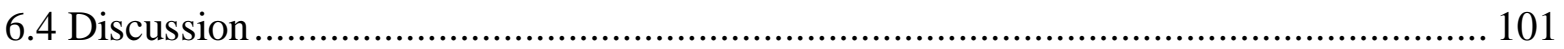

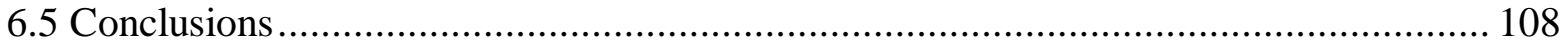

Section 3: Electrospray Ionization for the Analysis of Small Molecules ................................. 110

Chapter 7: Identification of a Metabolite of Nicotinylalanine using LC-ESI-MS.................. 111

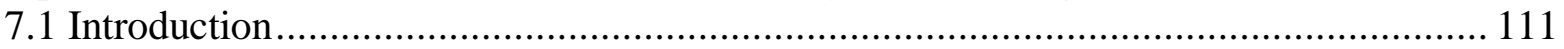

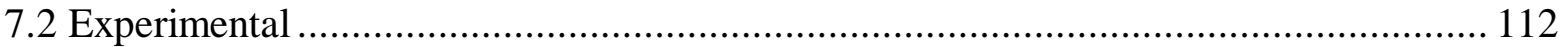

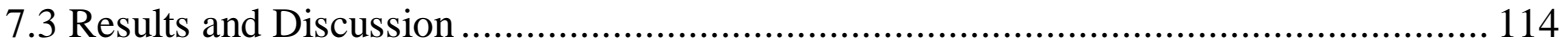

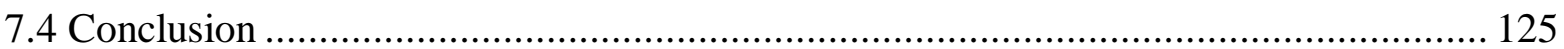

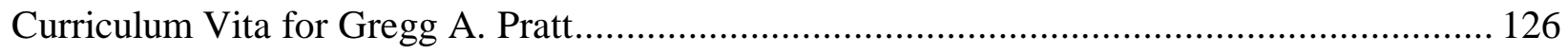

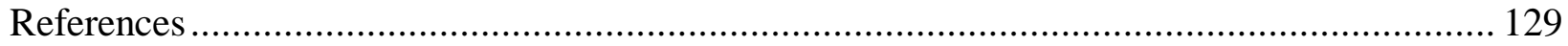




\section{Table of Figures}

Figure 1.2.1: Schematic of the Process of Electrospray Ionization......................................... 9

Figure 1 3.1: Schematic of an Ion Trap ....................................................................... 12

Figure 1.3.2: Schematic of the Finnigan LCQ Ion Trap Mass Spectrometer .......................... 15

Figure 2.1.1: Structure of the Heme Group in Myoglobin and Cytochrome c........................ 20

Figure 2.2.1: Ball and stick representation of Cytochrome c......................................... 21

Figure 2.2.2: Coordination About the Central Iron Atom in Cytochrome c.............................. 22

Figure 2.2.3: Points of Attachment of Protein to Porphyrin in Cytochrome c. ......................... 23

Figure 2.3.1: Coordination About the Heme Iron in Myoglobin. .................................... 24

Figure 2.3.2: Oxygen Binding in Myoglobin................................................................. 25

Figure 2.3.3: Heme Unit (red) at the surface of Myoglobin .............................................. 26

Figure 2.6.1: Charge State Distribution of Cytochrome $\mathrm{c}$ in deionized water......................... 32

Figure 3.4.1: Mass spectrum of cytochrome $\mathrm{c}$ in $20 \%$ acetonitrile, capillary temperature $=150^{\circ} \mathrm{C}$.

Figure 3.4.2: Mass spectrum of cytochrome $\mathrm{c}$ in $20 \%$ acetonitrile, capillary temperature $=200{ }^{\circ} \mathrm{C}$

Figure 3.4.3: Mass spectrum of cytochrome $\mathrm{c}$ in $20 \%$ acetonitrile, capillary temperature $=250{ }^{\circ} \mathrm{C}$

Figure 3.4.4: Mass spectrum of cytochrome $\mathrm{c}$ in $60 \%$ acetonitrile, capillary temperature $=150^{\circ} \mathrm{C}$

Figure 3.4.5: Mass spectrum of cytochrome $\mathrm{c}$ in $60 \%$ acetonitrile, capillary temperature $=200^{\circ} \mathrm{C}$.

Figure 3.4.6: Mass spectrum of cytochrome $\mathrm{c}$ in $60 \%$ acetonitrile, capillary temperature $=250^{\circ} \mathrm{C}$.

Figure 3.4.7: Mass spectrum of cytochrome $\mathrm{c}$ in $20 \%$ acetonitrile, tube lens $=0 \mathrm{~V} \ldots \ldots \ldots \ldots \ldots . . . .49$

Figure 3.4.8: Mass spectrum of cytochrome $\mathrm{c}$ in $20 \%$ acetonitrile, tube lens $=30 \mathrm{~V} \ldots \ldots \ldots \ldots . . .50$

Figure 3.4.9: Mass spectrum of cytochrome $\mathrm{c}$ in $20 \%$ acetonitrile, tube lens $=60 \mathrm{~V}$.............. 51

Figure 3.4.10: Mass spectrum of cytochrome $\mathrm{c}$ in $60 \%$ acetonitrile, tube lens $=0 \mathrm{~V}$...............52

Figure 3.4.11: Mass spectrum of cytochrome $\mathrm{c}$ in $60 \%$ acetonitrile, tube lens $=30 \mathrm{~V} \ldots \ldots \ldots \ldots . . .53$

Figure 3.4.12: Mass Spectrum of cytochrome $\mathrm{c}$ in $60 \%$ acetonitrile, tube lens $=60 \mathrm{~V} \ldots \ldots \ldots \ldots . . . .54$

Figure 3.4.13: Average Observed Charge vs. Tube Lens Voltage for Cytochrome c in 0, 20, and

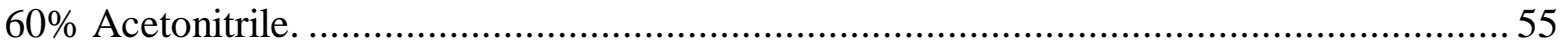

Figure 3.4.14: Interday Variability of Tube Lens Settings ............................................ 56

Figure 4.3.1: Mass Spectrum of Cytochrome c, Acetonitrile Sheath Liquid Flow Rate $=0 \mu 1 / \mathrm{min}$.

Figure 4.3.2: Mass Spectrum of Cytochrome c, Acetonitrile Sheath Liquid Flow Rate =20 $\mu 1 / \mathrm{min}$.

Figure 4.3.3: Mass Spectrum of Myoglobin, Acetonitrile Sheath Liquid Flow Rate $=0 \mu 1 / \mathrm{min} .66$ Figure 4.3.4: Mass Spectrum of Myoglobin, Acetonitrile Sheath Liquid Flow Rate $=5 \mu 1 / \mathrm{min}$. . 67 Figure 4.3.5: Mass Spectrum of Myoglobin, Acetonitrile Sheath Liquid Flow Rate $=10 \mu 1 / \mathrm{min} .68$ Figure 4.3.6: Mass Spectrum of Myoglobin, Acetonitrile Sheath Liquid Flow Rate = 20 $\mu 1 / \mathrm{min} .69$ Figure 4.3.7: Mass Spectrum of Myoglobin, Acetonitrile Sheath Liquid Flow Rate = 30 $\mu 1 / \mathrm{min} .70$ 
Figure 4.3.8: Average Observed Charge of Myoglobin and Apomyoglobin

vs. Acetonitrile Sheath Liquid Flow Rate

Figure 4.3.9: Percentage of Response Due to Myoglobin and Apomyoglobin at Various

Acetonitrile Sheath Liquid Flow Rates

Figure 5.3.1: Heme Ion Intensity versus \% Relative Collision Energy for the 9+ to 13+ Charge

States of Myoglobin

Figure 5.3.2: Heme Ion Intensity versus \% Relative Collision Energy for the 7+ to 11+ Charge

States of Cytochrome c.

Figure 5.3.3: Heme Ion Intensity versus \% Relative Collision Energy for the 9+ to 11+ Charge

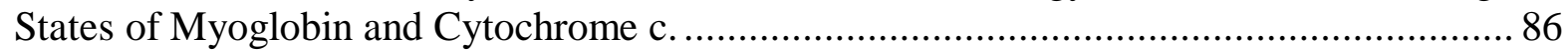

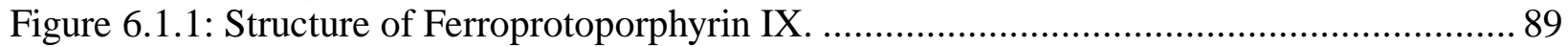

Figure 6.1.2: Coordination About the Central Iron Atom in Cytochrome c.............................. 90

Figure 6.1.3: Coordination About the Heme Iron in Myoglobin. ....................................... 91

Figure 6.1.4: Oxygen Binding in Myoglobin................................................................... 92

Figure 6.3.1: Mass Spectrum of Cytochrome c ........................................................... 93

Figure 6.3.2: MS2 Spectrum of the 9+ Charge State of Cytochrome c................................... 94

Figure 6.3.3: MS3 Spectrum of m/z $1374(9+) / 617$ for Cytochrome c................................. 95

Figure 6.3.4: MS4 Spectrum of 8+/617/544 for Cytochrome c. ........................................ 95

Figure 6.3.5: MS4 Spectrum of 9+/617/557 for Cytochrome c. ......................................... 96

Figure 6.3.6: Full Mass Spectrum of Myoglobin ............................................................ 97

Figure 6.3.7: MS/MS of the 9+ Charge State of Holomyoglobin ..................................... 98

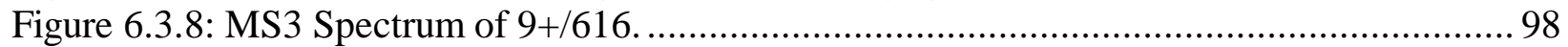

Figure 6.3.9: MS4 of 9+/616/557 for Myoglobin. ......................................................... 99

Figure 6.3.10: MS4 of 9+/616/544 for Myoglobin..................................................... 100

Figure 6.4.1: Observed Fragmentation of the Heme Ions from Myoglobin and Cytochrome c. 101

Figure 6.4.2: Comparison of MS4 of m/z 557 Spectra for Cytochrome $\mathrm{c}$ and Myoglobin ........ 103

Figure 6.4.3: Comparison of the MS4 of 544 Spectra for Cytochrome c and Myoglobin........ 104

Figure 7.3.1: Selected Ion Chromatogram of m/z 195 and Mass Spectrum of Nicotinylalanine

(NAL).

Figure 7.3.2: Selected Ion Chromatogram of m/z 237 and Mass Spectrum of Synthetic N-

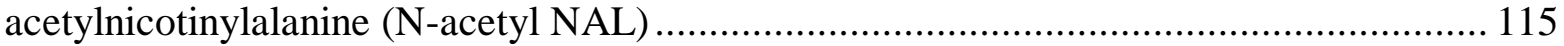

Figure 7.3.3: Chromatogram and Mass Spectrum of the Urine Sample Extract .................... 116

Figure 7.3.4: MS/MS Spectrum of Nicotinylalanine ..................................................... 117

Figure 7.3.5: MS/MS spectrum of Synthetic N-acetyl NAL ............................................ 118

Figure 7.3.6: MS/MS of Urine Sample Extract ............................................................ 119

Figure 7.3.7: MS3 of 237, 178 for Synthetic N-acetyl NAL .......................................... 120

Figure 7.3.8: MS3 of 237, 178 for Urine Sample Extract ............................................ 121

Figure 7.3.9: Proposed structures of daughter ions of NAL ........................................... 123

Figure 7.3.10: Proposed structures of daughter ions of $\mathrm{N}$-acetylNAL ............................... 124

Figure 7.4.1: Proposed Metabolism of NAL ............................................................... 125 


\section{Table of Tables}

Table 3.2.1: Instrument Parameters and Setpoints Evaluated. 38 Table 3.4.1: Influence of Heated Capillary Temperature on the Average Observed Charge (AOC) for the three tested solutions.

Table 4.3.2: Influence of Tube Lens Voltage on the AOC's of Cytochrome c. 48

Table 7.3.1: Comparison of mass specrometric data for $N$-acetylNAL standard and the Urinary Metabolite. Values in parentheses are \% relative abundance 122. 
Section 1: Electrospray lonization Mass Spectrometry 


\section{Chapter 1: An Introduction to Electrospray lonization Mass Spectrometry}

\section{1: A Brief History of Mass Spectrometry}

The behavior of charged particles in the gas phase has long intrigued scientists. From the discovery of "positive rays of electricity" by Thompson ${ }^{1}$ to the present use of modern mass spectrometers, the analyses of ions in the gas phase have made significant contributions to our understanding of the natural world. Early mass spectrometers were responsible for the discovery of elemental isotopes, and later designs affording enhanced resolving power were used to make accurate mass measurements that helped to establish the modern periodic table of elements. These early fundamental contributions to basic science made by mass spectrometry helped to establish the discipline. Since then, the use of mass spectrometers has grown such that the instrumentation is now widely and routinely applied in fields as diverse as materials science, chemistry, biology, biochemistry, and pharmacology. Significant developments in technology have enabled mass spectrometry, once an obscure discipline in the hands of a few chemists, physicists, and engineers to become the analytical tool of choice for a diverse range of applications

The developments that have allowed mass spectrometry to flourish in the later part of the twentieth century have been necessitated by the growth in separation sciences, specifically the need for selective detection devices. The advances in mass spectrometry instrumentation that have allowed these systems to be so widely applied included improvements in the speed and reliability of mass analyzers, and the development of more versatile ionization sources.

All early mass analyzers suffered from a common problem: slow speed. Until the development of transistors in the 1950's and 1960's, analytical instruments of all types were limited by the ability of the electronics to accurately measure time. Even early Time of Flight 
mass analyzers, relatively simple instruments in which the mass of an ion is calculated based upon the amount of time it takes for the ion to transit a flight tube, suffered due to slow electronics. The electronics available would not allow for accurate discrimination between similar masses. And magnetic sectors are inherently slow-scanning, so available mass analyzers up until the 1970's were limited by their ability to resolve similar masses, or by their ability to conduct rapid, accurate mass analyses. This limitation was particularly problematic in chemical separations, as rapid response times are required to distinguish one chemical species from another.

Therefore with the development of fast-response electronics, the applicability of mass spectrometric techniques improved. However the growth of the separation sciences and particularly the use of Gas Chromatography (GC) required that mass spectrometers utilize a continuous ion source that is compatible with separation techniques. Many of the early ion sources were of a pulsed design. For instance, in a Spark Source (SS), a current passed between electrodes volatilizes and ionizes a solid material, and the ion generated then transits a magnetic sector mass spectrometer. Since it is known when the spark was fired, the detector signal corresponding to each firing of the source can readily be deduced. However since chromatographic elution profiles are transient events of varying duration, a source was needed that continuously produced ions, and mass analyzers were required to rapidly distinguish between ions that are continuously produced.

The need for a continuous ion source was met in the development of the electron impact (EI) ion source. In this source a current applied to a metal filament produces a beam of thermally emitted electrons that are given direction by an electric field. The vapor effluent from a GC is directed into the electron beam, and upon impact of the electron beam upon the analytes, ions are 
formed. This source is readily interfaced to a mass spectrometer, as similar operating pressures are required. The mechanism of ionization is highly energetic, and much fragmentation is observed. While this is ideal for structural elucidation, sensitivity is poor due to extensive fragmentation, and little or no molecular weight information is conveyed as the molecular species are destroyed by the ionization process.

Another ionization source sought to alleviate some of these problems. Developed by Munson and Field ${ }^{2}$ in the 1960's, the Chemical Ionization (CI) source utilized the same beam of ions as the EI source, but in this case the electrons impinged upon a stream of ionizeable gas in the ion source. The electrons ionized the gas, which in turn transferred charge to the analytes in the GC effluent, thereby ionizing molecules of interest in the carrier gas stream. This proved to be a much softer method of ionization as little analyte fragmentation occurred, and thus sensitivity was improved and molecular weight information could be better conveyed.

Applicability was still limited though, by two critical factors. As GC elution profiles are very narrow, mass analyzers were required which could quickly scan a relatively large mass range while preserving adequate mass resolution. This call was answered by the development of Quadrupole Mass Analyzers and Quadrupole Ion Traps. Quadrupole Mass Analyzers (QMA) function by applying both RF and DC fields to a series of four metal rods. The DC applied to the rods in the x-z plane acts as a high pass mass filter, as it deflects ions of low mass outside of the radius and they are filtered out. Ions of high mass are little affected by this field, and thus transit the rods and generate a signal at the detector. The RF field has the opposite effect, as small ions can more readily follow the field oscillations, and high-mass ions are more prone to get lost in the RF field. Thus the RF field acts as a low pass filter. When the two fields work together, only ions of a narrow mass range can maintain a stable orbit within the analyzer resulting in a signal 
at the detector. The Quadrupole Mass Analyzer revolutionized mass spectrometry, as it affords unit resolution, an upper mass limit of $4000 \mathrm{AMU}$, and can rapidly scan a large mass range. These analyzers are still the choice for analyses requiring high sensitivity and reproducibility. They are inexpensive to produce, and may be reproducibly manufactured. These qualities of the QMA allowed a rapid expansion of mass spectrometry throughout the 1980's, and QMA-based mass spectrometers began to find their way into pharmaceutical and environmental laboratories. Quadrupole Ion Traps will be discussed in detail in Section 1.3 of this chapter.

With the growth of biotechnology in the 1980's, a need arose for reliable methods of analyzing biopolymers such as proteins and oligonucleotides. The chemical properties of these analytes required that a new technology be developed. While Gas Chromatography works quite well for relatively small, volatile analytes, biopolymers are neither small nor volatile. Proteins often possess molecular weights in excess of 100 kilodaltons, In addition to their large size, the presence of polar functional groups within their structures make proteins extremely non-volatile. Proteins are also thermally labile, rapidly decomposing upon exposure to heat. Derivatization chemistries developed to make peptides more volatile for analysis by GC were of some benefit, but the reagents are costly, the procedures time consuming, and at times, the results are unreliable. Although a derivatization may have succeeded in the analysis of a peptide, the thermal lability of intact proteins was still problematic. It was clear that a new method of introducing ions to the gas phase from the solution environment was required, and particularly one that would allow for the analysis of proteins and peptides separated by Liquid Chromatography (LC).

One solution to the problem of ion transport to the gas phase from solution was developed by Vestal ${ }^{3}$ at the University of Houston in the early 1980's. The Thermospray ion 
source utilized a series of pumps to step down from a high-pressure region wherein Liquid Chromatography effluent was delivered. Analytes could be ionized by CI, or by an energetic plasma generated by an electrode in a region of low pressure separated from the LC effluent by a skimmer. Though it was a versatile source, it required maintenance, and LC flow rates were extremely limited.

The development of the MAGIC (Monodispersed Aerosol Generation Interface for Chromatography) by Browner ${ }^{4}$ in the mid-1980's sought to address the problem of limited flow rate that plagued the Themospray ion source. Here a stream of helium placed orthogonally to the LC effluent line created an aerosol. A portion of the aerosol was then drawn through a series of skimmers into regions of high vacuum. Behind the skimmers a region of supersonic expansion created a shock wave, the energy of which served to fragment large molecules. The stream was then directed toward an EI or CI ion source where ionization was accomplished, and then into the mass spectrometer. The MAGIC source did afford better compatibility with Liquid Chromatography, as flow rates up to $0.5 \mathrm{ml}$ per minute were permissible. But it was not particularly good for large molecules, as much fragmentation took place in the shock wave. Although MAGIC was successful as it represented the first truly useful ion source with compatibility with LC, developments were on the horizon that would supplant this source as the ionization method of choice for LC/MS applications.

The advent of Electrospray Ionization (ESI) by Fenn and others in $1989^{5}$ created a revolution in the analysis of both proteins and small molecules. The ability of this method to deliver large, thermally labile biomolecules to the gas phase intact made possible the analysis of proteins that were formerly experimentally inaccessible. Since its development, electrospray has become the ionization method of choice for the analysis of proteins ${ }^{6}$, oligosaccharides ${ }^{7}$, 
oligonucleotides $^{8}$, and other biopolymers that have historically been difficult to analyze. To date, electrospray ionization has been employed in protein sequencing ${ }^{9}$, conformational analysis $^{10}$,

gas-phase reactivity studies ${ }^{11}$, determining the oxidation states of metals in metalloproteins ${ }^{12}$, and in numerous other protein-related applications. And due to its compatibility with Liquid Chromatography, it has been the most widely applied ion source for LC/MS applications, particularly for pharmaceutical analyses requiring high sensitivity.

\subsection{Electrospray lonization}

The process of electrospray has its roots in the work of John Zeleny, a physicist at Yale University in the early part of the twentieth century who performed pioneering research on the electrostatic dispersion of liquids into charged droplets ${ }^{13}$. Malcolm Dole first applied the concept to macromolecules in the 1960's in the analysis of polystyrene oligomers ${ }^{14}$. The first application to proteins came in the early 1980's with groundbreaking work by Fenn at Yale University. Fenn's work marked the first interfacing of an electrospray ion source to a mass spectrometer, and the first observation of the multiple-charging characteristic of proteins analyzed by ESI-MS. Since Fenn's application of this method to the analysis of proteins, the technique has been widely applied in the separation, sequencing, and quantification of known proteins, as well as in the determination of molecular mass and identification of unknown proteins.

The fundamental challenge in transferring ions from the condensed phase to the gas phase is in overcoming the energy barrier between the solvent environment, wherein ions are stabilized by strong interactions with solvent molecules and other ions and the gas phase, wherein such stabilizing forces are greatly diminished. In order then to transfer an ion to the gas phase, interactions with other ions and molecules in the condensed phase must be disrupted. In 
order to accomplish this, a substantial amount of energy must be invested, making the transfer of ions to the gas phase a strongly endothermic process. Sufficient energy to disrupt solvation can be delivered rapidly by thermal heating. However rapid heating often imparts sufficient energy as to cause bonds to break, and dissociation of the species of interest may result. This approach would then not be feasible for the introduction of thermally labile molecules such as proteins to the gas phase, as extensive fragmentation would ensue upon rapid heating.

The thermal energy delivered during the process of electrospray is delivered gradually, and at low temperatures. Because the required energy is delivered more slowly and the temperatures at which desolvation is achieved are moderate, little disruption of bond occurs and thus molecular fragmentation is minimal, even in the case of thermally labile biomolecules. For this reason electrospray is termed a "soft" method of ionization ${ }^{24}$. 


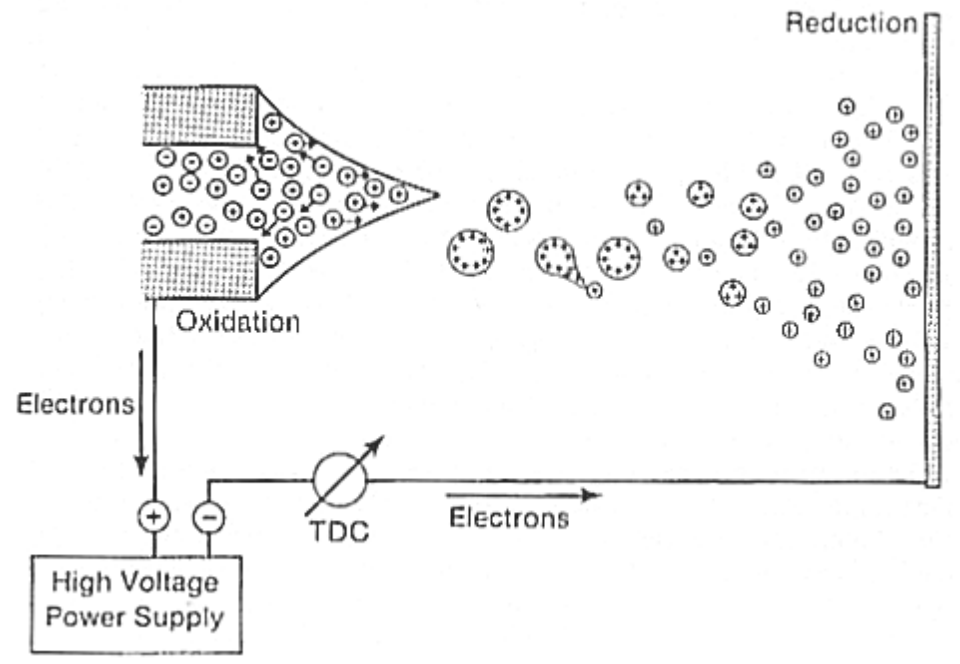

Figure 1.2.1: Schematic of the process of electrospray ionization ${ }^{24}$

A schematic of the electrospray process is seen in Figure 1.2.1. A high voltage power supply supplies a voltage of $2-4.5 \mathrm{kV}$ to a stainless steel capillary. The counter-electrode, spaced a few centimeters away from the capillary, is held at ground or near ground potential. The counter-electrode in commercial ESI-MS systems also serves as the orifice for sample introduction into the mass spectrometer. The thinness of the capillary creates a very high electric field at the capillary tip, as is outlined in the following equation ${ }^{24}$

$E_{c}=\frac{2 V_{c}}{r_{c} \ln \left(4 d / r_{c}\right)}$

Where

$E_{c}=$ electric field at capillary tip

$r_{c}=$ radius of capillary

$d=$ distance between capillary and counter - electrode

$V_{c}=$ voltage applied to capillary tip 
Using this equation, and assuming that $4 \mathrm{kV}$ is applied to a $10^{-4} \mathrm{~m}$ capillary tip that is separated from the counter-electrode by a distance of $0.03 \mathrm{~m}$, a field of approximately $10^{7}$ volts per meter is generated at the capillary tip. This field serves to repulse positive ions in solution, which move to the tip of the capillary toward the meniscus of the liquid filling the capillary, creating an excess of positive charges at the tip of the capillary. Each positive charge is repulsed by the imposed field, the sum of which is sufficient to overcome the surface tension of the liquid within the capillary. This causes expansion of the surface of the liquid that allows the positive charges to move further away from the capillary tip in order to escape the field. This forces the surface of the liquid into a funnel-shaped structure termed a "Taylor cone"15. The tip of the cone continues to extend toward the counter-electrode until the stability of the cone breaks down, and the liquid is dispersed into a series of charged droplets.

In commercial ESI-MS systems, a stream of nitrogen sheath gas is delivered co-axially around the outside of the capillary. The sheath gas serves to create an aerosol of charged liquid as the droplets emerge from the Taylor cone. The flow of nitrogen also begins to shrink the droplets by evaporation. As the droplets shrink, the positive charges within the droplets become more closely packed, and repulsive forces within the droplet increase. When the forces of repulsion within a given droplet exceed the surface tension of the droplet (the Raleigh limit) the droplet fissions, producing many droplets of smaller size. This process is repeated until the droplets are approximately 10 nanometers in diameter.

The process by which ions in the gas phase are formed from the very small charged droplets is a question of some debate, and two models have been developed to explain this phenomenon. In the Ion Evaporation Model (IEM) espoused by Iribane and Thomson ${ }^{16}$, the charged ions are emitted directly from the very small (radius $<10 \mathrm{~nm}$ ) droplets. This model 
suggests that when the droplets are sufficiently small, the repulsive forces imposed by charges remaining in the droplet are sufficient to eject an escaping ion. Therefore Raleigh fissions continue only to a critical point, at which time ejection of ions from the droplets becomes the dominant mechanism. The competing model, formulated by Dole and Rollgen ${ }^{17}$ and known as the Charge-Residue Model (CRM), suggests that fissions continue until only one solute molecule resides in each charged droplet, and it becomes an ion by accepting charge from the remaining solvent as it evaporates. Therefore according to the Charge Residue Model, fission of droplets is the dominant process throughout the process of electrospray. While both models explain some of the mass spectrometric data observed, neither is universally accepted. Research continues to this date to determine the exact nature of the mechanism of electrospray ionization.

\subsection{Ion Trap Mass Spectrometry}

Van Berkel and co-workers accomplished the first successful marriage of an electrospray ionization source to an ion trap mass spectrometer in $1990^{18}$. In interfacing an electrospray ion source to an ion trap mass spectrometer, a powerful platform for protein analysis was developed. Because of its flexibility, the ion trap is now widely applied in protein conformational analysis and kinetics. And the ability to store ions in a confined space and to perform a theoretically unlimited number of stages of MS has allowed the ion trap to be utilized in protein sequencing, and both small molecule and protein structural determination.

The ion trap was first described by Wolfgang Paul in $1953^{19} 20$, and the three electrode arrangement he proposed for its construction was awarded a German patent in $1956^{21}$. The significance of this achievement was recognized by the award to Paul of a share of the 1989 Nobel Prize in Physics 
Ions are retained within an ion trap by a combination of radio frequency $(\mathrm{RF})$ and direct current fields. Figure 1.3.1. shows a schematic of a generalized ion trap. A RF field is applied to the ring electrode, a circular electrode that forms the constricted middle of the ion trap. At the ends of the trap are the endcap electrodes, which serve to restrain the ions in the $\mathrm{z}$-direction.

Oscillations in the RF field impart to the stored ions a circular motion in the $\mathrm{x}-\mathrm{y}$ plane within the trap, and the DC voltages applied to the endcaps limit motion in the z-direction. Therefore the combination of electrodes which constitute the trap cooperate to limit motion of the ions to a confined space within the trap.

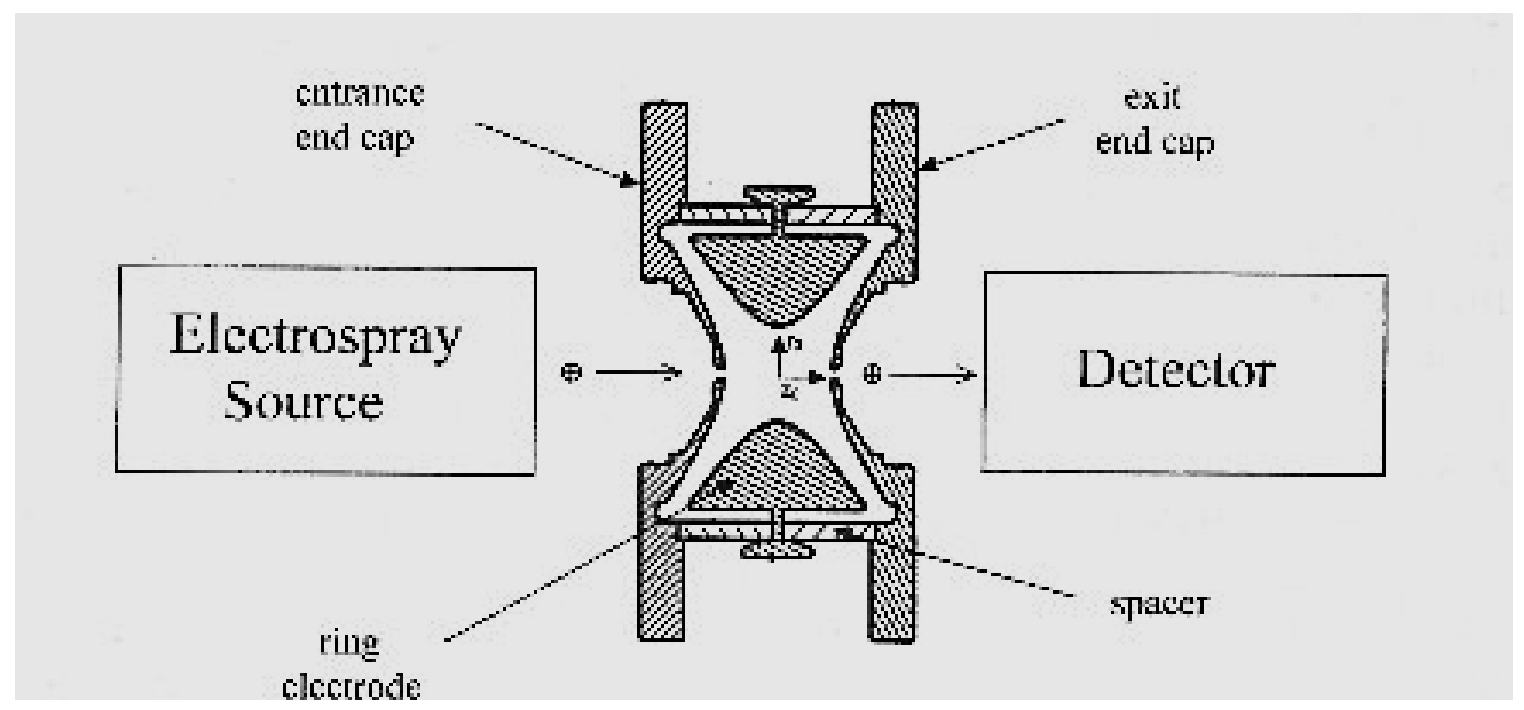

Figure 13.1: Schematic of an Ion Trap ${ }^{24}$. 
Once restrained within the trap, the collected ions must be ejected from the trap for detection to occur. The procedure for ejecting ions from the trap relies upon the principle of a mass-selective instability scan developed by Stafford in $1983^{22} 23$. While the ability to trap ions in a combination RF-DC field had been known since the work of Paul, Stafford's discovery made the ion trap a useful analytical tool. In order for ions to be ejected from the trap and be detected, their orbit within the trap must be disturbed. Without some perturbation of their motion within the trap, the ions will continue to cycle within the confined space. Stafford observed that by ramping the RF applied to the ring electrode, instabilities were created in the motion of the ions, and they could be ejected from the trap for detection. Of tremendous analytical significance is the fact that in increasing the RF, ions of increasing mass are removed from the trap in succession. That is, in ramping the RF voltage applied to the ring electrode, ions are scanned out of the trap from lowest mass to highest mass. Thus in calculating at what RF setting a given detector signal was observed, the mass of the ion could be determined. It is clear that the work of Stafford in determining the mathematics of stability and instability in an ion trap made a vital contribution that allowed for the development and implementation of today's modern ion trap.

Within the trap, a small amount of helium present serves to dampen ion motion and minimize ion collisions that would otherwise cause fragmentation and expulsion of ions from the trap. The benefits of the presence of a damping gas were discovered quite accidentally. In the early 1980's, researchers at Finnigan Corporation were striving to develop the ion trap as a selective detector for Gas Chromatography. At this time, the approach was to deliver the GC effluent directy to the ion trap, wherein ionization would be carried out. Therefore the GC capillary column was plumbed directly into the ion trap. The carrier gas used for the GC analysis was helium, and Stafford ${ }^{23}$ observed that marked improvements to mass resolution and 
sensitivity were achieved when the helium was present within the trap. This is thought to result from effective cooling of the ions as they impart energy to the helium nuclei, and the subsequent settling of ions into dense packets at the center of the trap that results in more effective mass scanning and increased signal intensity. ${ }^{24}$

The helium also functions as a reagent for Collision Induced Dissociation (CID). When a small voltage is applied to the endcap electrodes, the contents of the ion trap gain kinetic energy. This energy is translated into molecular internal energy via collisions of ions with helium nuclei within the trap. Numerous collisions impart sufficient internal energy to the ions that they begin to fragment, breaking into smaller pieces. The resulting fragment ions can then be analyzed by performing a mass-selective instability scan. Thus in this fashion, MS/MS is performed within the ion trap. Since the reactions occur in a confined space wherein the products may be retained, the number of stages of MS that can be performed on an ion trap is theoretically unlimited. The capability of ion traps to perform an unlimited number of stages of mass spectrometry makes this instrumentation a powerful tool in structural analysis. 


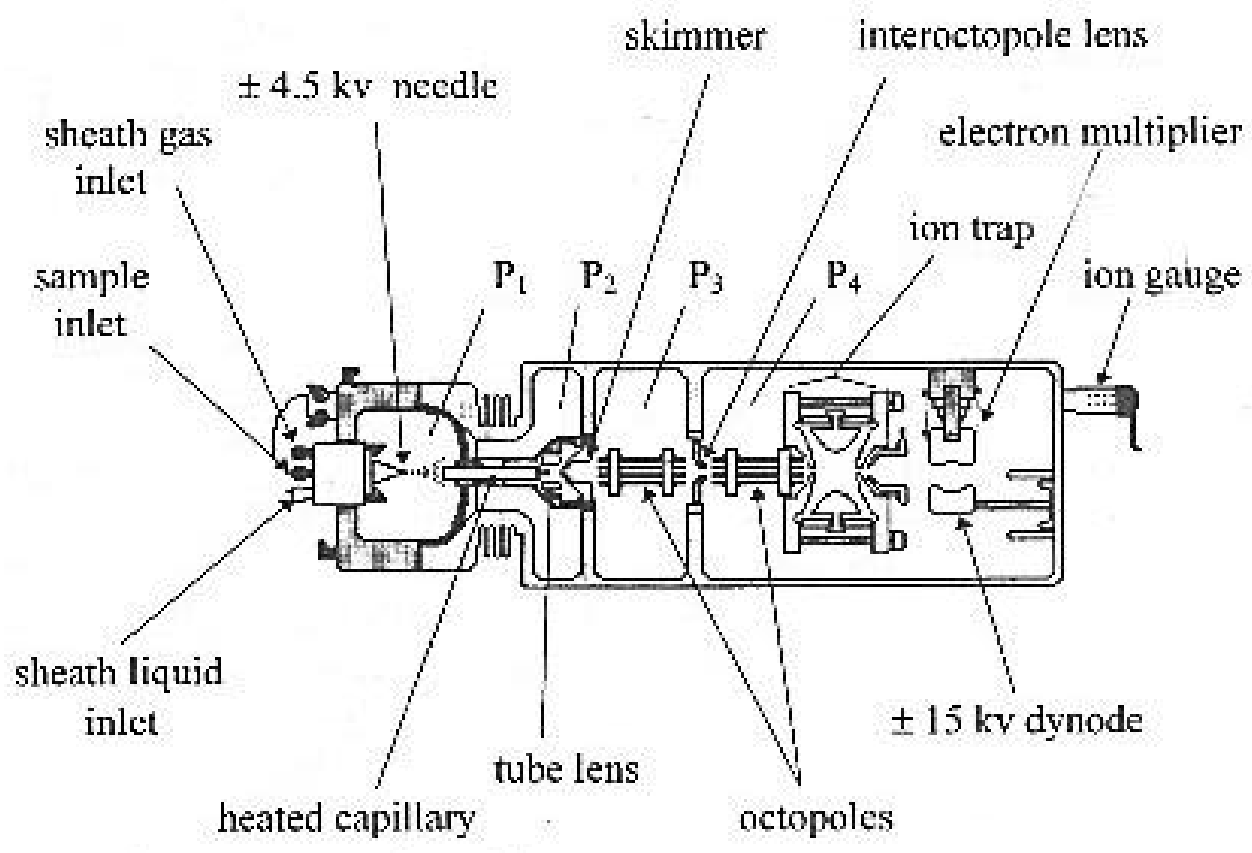

Figure 1.3.2: Schematic of the Finnigan LCQ Ion Trap Mass Spectrometer ${ }^{24}$

Figure 1.3.2 shows a schematic of the Finnigan LCQ ${ }^{\circledR}$ ion trap mass spectrometer used in all protein conformational analysis studies that constitute this dissertation. Solutions are electrosprayed at atmospheric pressure from a stainless steel needle at $4 \mathrm{kV}$ in pressure region 1 (P1). A dual-port turbomolecular pump creates successively higher vacuum in pressure regions 2-4, stepping the pressure down along the ion trajectory from 760 Torr in P1 (atmospheric pressure) to $10^{-5}$ Torr in $\mathrm{P} 4$. The process of desolvation is begun by a steam of nitrogen flowing coaxial to the electrospray needle, which forms an aerosol of charged droplets. The aerosol is sampled by a heated capillary tube maintained at approximately $200^{\circ} \mathrm{C}$, the elevated temperature of which completes the process of desolvation. The ions then emerge from the capillary tube, and 
move through a skimmer to a region of lower pressure. The ion beam that emerges from the skimmer is focused by a tube lens into the first of two sets of RF-only ion guides Ion guides were chosen for this instrument because they provide better ion transmission than conventional lens sytems, particularly ions of low mass, as scattering is reduced. ${ }^{25}$ Am inter-octapole lens separates the two sets of octapole rods. In addition to its primary role in ion transmission and injection, its location on the manifold wall between regions P3 and P4 allows the inter-octapole lens to serve as a divider between regions of differing pressures, which aids in differential pumping. When suitable RF fields are applied to the octapole ion guides, ions are injected into the trap through perforations in the entrance endcap electrode. During ion injection and accumulation, RF power is applied to the octapole rods. When ions are being scanned out of the trap or ion isolation is being performed, the RF is turned off and ions are not injected into the trap ions then enter the trap via a perforation in the endcap

Upon ejection from the trap, ions are drawn by electrostatic attraction to a $15 \mathrm{kV}$ dynode. Ions incident upon the dynode surface create secondary particles (electrons and ions) which are detected by an electron multiplier as is indicated in Figure 1.3.1. 
Section 2: Analysis of Proteins by Electrospray lonization/lon Trap Mass Spectrometry 


\section{Chapter 2: Electrospray lonization Mass Spectrometry for the Analysis of Proteins}

\subsection{Introduction to Protein Structure}

Proteins are large, complex macromolecules that are vitally important in cellular structure, energy generation and respiration, signal transduction, and a multitude of other functions. They may be composed of from one to many polypeptide subunits that make up the protein in its

entirety. The structure of proteins can be analyzed by way of a four-level hierarchy ${ }^{26}$ of organization that describes the relationship of constituent amino acid residues within the polypeptide subunits, the spatial relationship between neighboring amino acid residues within the same subunit, the spatial relationship of amino acid residues that are not closely spaced along the subunit backbone, and the arrangement in space of polypeptide subunits that constitute the complete protein.

A protein's primary structure may be defined as the linear sequence of constituent amino acids that make up the polypeptide chains. Peptide bonds are formed between adjacent amino acids within each subunit, creating a long chain of residues that compose the polypeptide subunits. Though the simplest level of protein structure, the arrangement of amino acids often dictates much about the higher order structure that in many proteins determines the function of the molecule in a biological system.

The next level of organization is local spatial arrangement, or secondary structure. This refers to the relationship in space of amino acid residues that are close together in the primary structure. Though the peptide groups alone are rigid, planar structures, some rotation is possible about the alpha carbons in the chains that make possible orientations in space that lend stability to the subunit. Twisting about the $\mathrm{C} \alpha$ bonds allows for the for the interaction of amino acid 
residues close together in the linear sequence that impart stability to the polypeptide. For instance, hydrogen bonding of peptide $\mathrm{C}=\mathrm{O}$ group of the nth amino acid in a sequence to the $\mathrm{N}$ H group of the $(n+4)$ residue in the sequence creates an $\alpha$-helix, the most common element of secondary structure.

Tertiary structure refers to the overall structure of a polypeptide subunit, including interactions of residues far apart in the primary sequence. This may include the aggregation of elements of secondary structure, such as the collection of helices that form a globin domain in heme proteins. Tertiary structure also encompasses the disposition of side chains connected to the polypeptide backbone. Tertiary structure, then, is the three-dimension shape of the entire

polypeptide, the conformation of which is dictated by the primary and secondary structures, as well as the interaction of elements of each that give shape to the polypeptide as a whole.

The quaternary level of protein structure refers to the spatial arrangement of polypeptide subunits that compose the protein. Nearly all of the known proteins possessing molecular weights greater than 100 kilodaltons are composed of more than one polypeptide subunit. It is the orientation of these constituent subunits in relation to one another that determines the quaternary structure of proteins.

\subsection{Introduction to Heme Proteins}

Heme proteins are an important class of macromolecules that play key roles in many significant biological processes. They are characterized by the presence of a porphyrin ring and one to several protein subunits. The iron atom at the center of the porphyrin may be found in the +2 or +3 oxidation state. This imparts to the protein abilities to serve as a center for electron transfer, to participate in oxidation-state dependent reversible oxygen binding, and to regulate biosynthesis and drug metabolism ${ }^{28}$. The redox capabilities of heme proteins therefore equip the 
molecule, and thus afford the cell, with unique properties that allow the heme proteins to participate in many functions that are vital to cellular function and vitality. Figure 2.1.1 shows the structure of ferroprotoporphyrin IX, the prosthetic heme group found in hemoglobin, myoglobin, and cytochrome c.

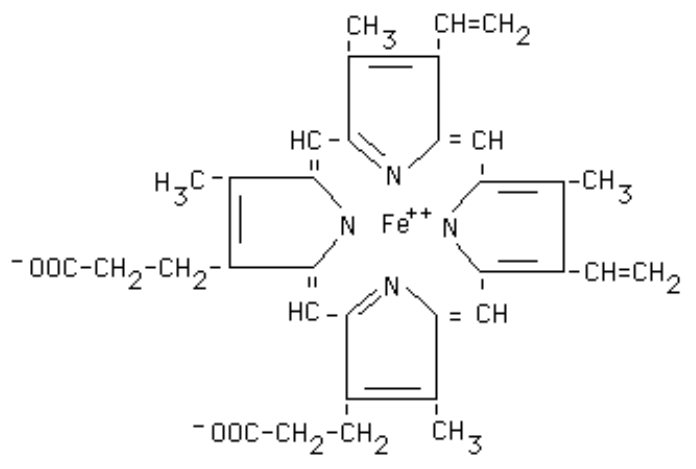

Figure 2.1.1: Structure of the Heme Group in Myoglobin and Cytochrome $\mathrm{c}^{30}$.

\subsection{Cytochrome c}

Cytochrome $\mathrm{c}$ is a $12 \mathrm{kDa}$ heme protein that is found in bacterial mitochondrial membranes. It resides within the mitochondrial wall where it may interact with numerous redox centers to shuttle electrons from center to center to facilitate metabolic reactions within the cell. In this protein the heme unit is close to the surface of the protein. Thus the iron atom at the center of the porphyrin structure is accessible to electron transfer. Cytochrome c plays a central role in mitochondrial respiration in bacterial cells, catalyzing electron transfer reactions within the organelle that are responsible for energy 
production within the cell ${ }^{29}$. Figure 2-2.1 shows a space-filling diagram of cytochrome $\mathrm{c}$. The iron-containing porphyrin near the molecular surface is shown in purple.

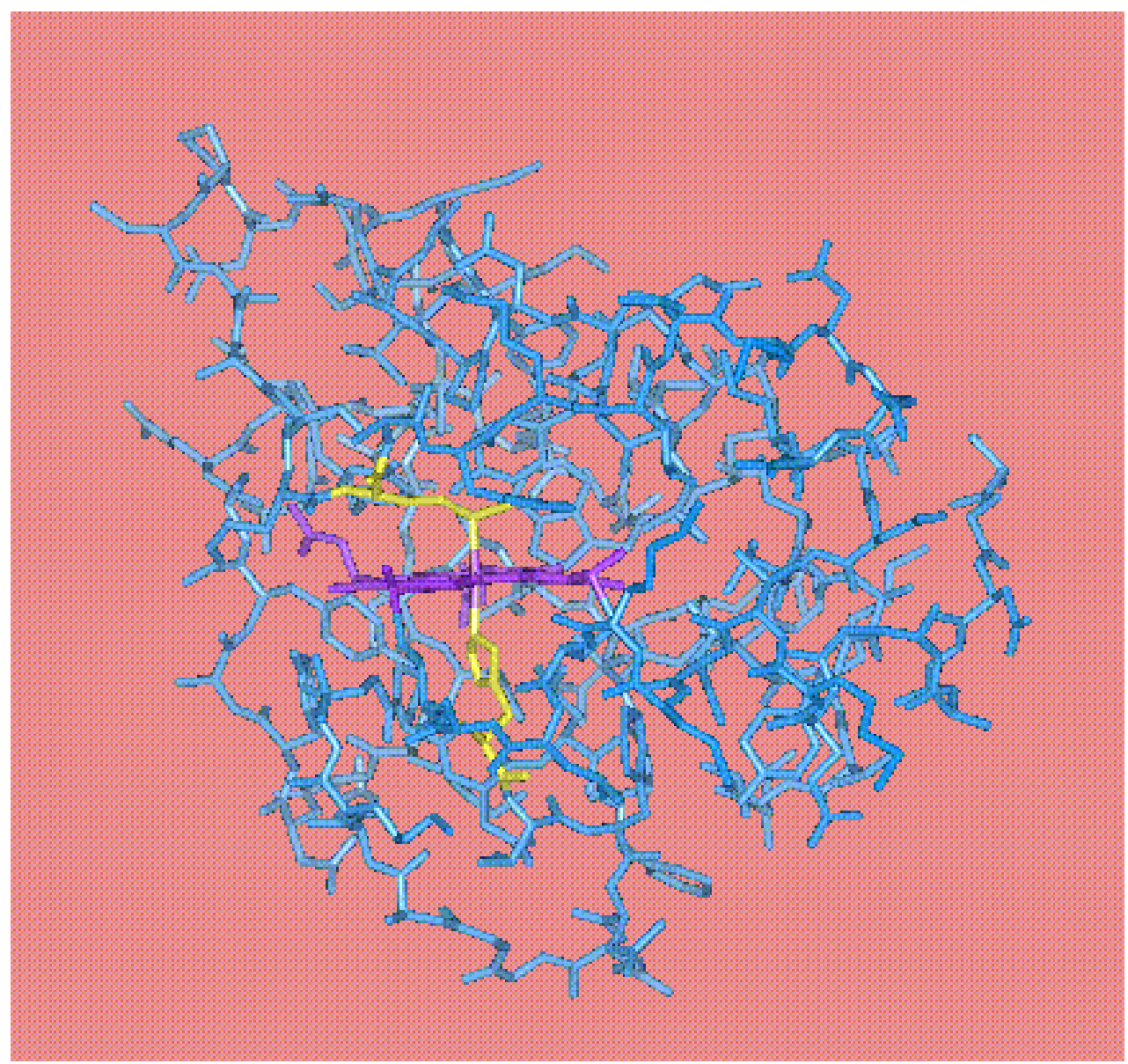

Figure 2.2.1: Ball and stick representation of Cytochrome $\mathrm{c}^{30}$.

In cytochrome c, coordination about the metal center is octahedral, with four coordination sites occupied by the porphyrin skeleton. The two remaining sites are occupied by histidine and methionine residues from the protein ${ }^{28}$. Figure 2.2.2 shows the coordination of the central iron atom in cytochrome c. 


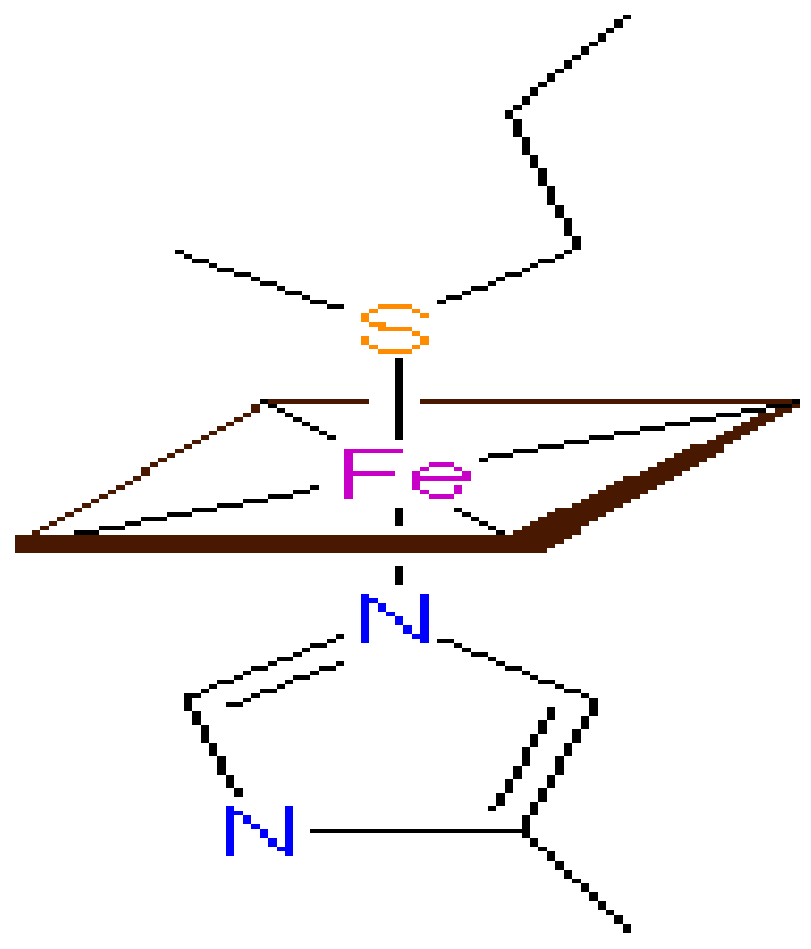

Figure 2.2.2: Coordination About the Central Iron Atom in Cytochrome $\mathrm{c}^{30}$.

In addition to the coordination sites, the porphyrin is connected to the protein by two cysteine bridges. As a post-translational modification, two thioether bonds are formed between the vinyl groups of the porphyrin and two cysteine sulfur groups of the protein subunit2. This arrangement imparts stability of position to the heme unit within the structure, and anchors the porphyrin at the molecular surface where it can be readily oxidized or reduced. Figure 2.2.3 shows the attachment of the porphyrin to the protein by two coordination sites to the central iron atom, and two covalent linkages between the protein and terminal portions of the porphyrin structure. 


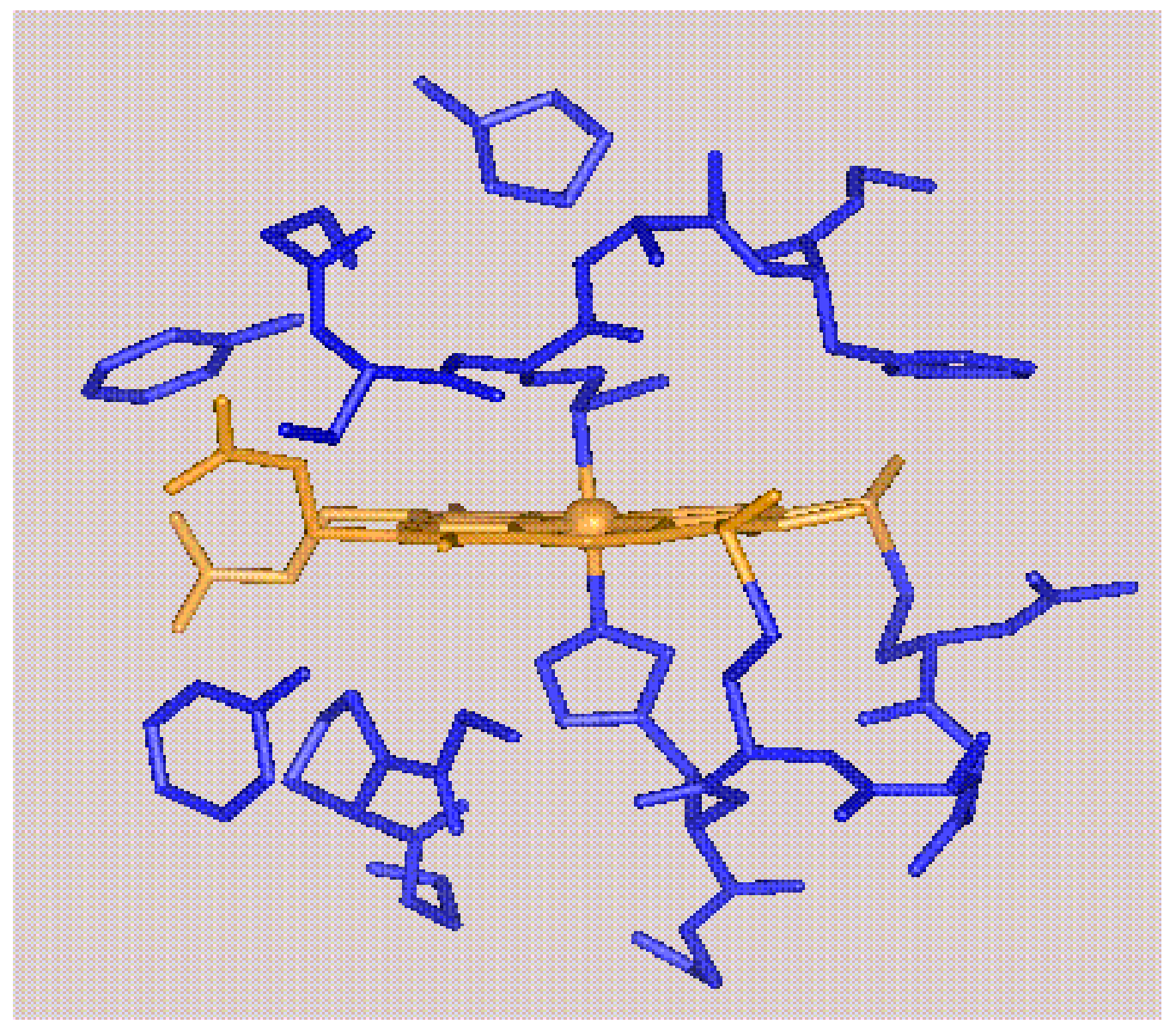

Figure 2.2.3: Points of Attachment of Protein to Porphyrin in Cytochrome $\mathrm{c}^{30}$.

\subsection{Myoglobin}

In another group of heme-containing proteins, the globins, the coordination about the central iron atom in the porphyrin differs from that found in the cytochromes. In the globin class of heme proteins that contains myoglobin and hemoglobin, the arrangement about the iron center is 5-coordinate. As in the cytochromes, a histidine residue of the protein occupies an axial coordination site, and the equatorial sites are coordinated to the nitrogen atoms at the center of the porphyrin core ${ }^{31}$. Figure 2.3.1 shows the coordination about the iron atom in myoglobin. 


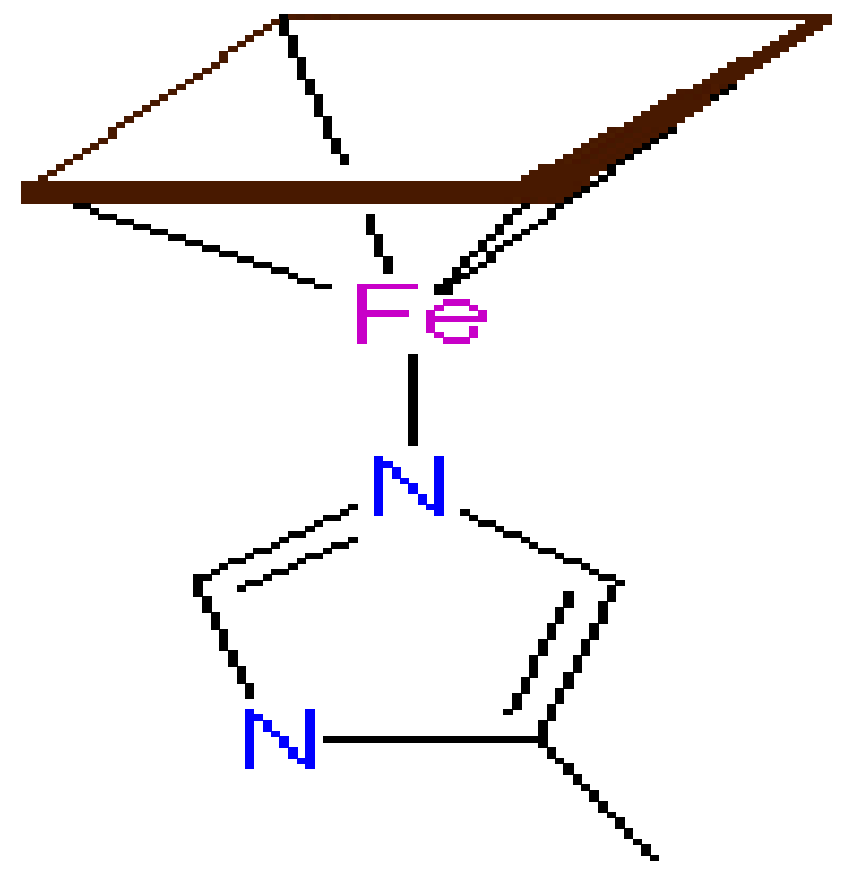

Figure 2.3.1: Coordination About the Heme Iron in Myoglobin ${ }^{30}$.

However unlike in the cytochromes, the remaining axial site is unoccupied in the globin heme proteins. This axial site is used to reversibly bind oxygen, thus these proteins function in the transportation and storage of molecular oxygen.

When the iron is in the oxidized state, the d5 electron configuration allows for the binding of the vacant axial site to dioxygen. When the molecule is reduced to the +2 oxidation state, the oxygen is released and the metal is then oxidized and the cycle is repeated. Figure 2.3.2 shows a schematic of the binding process. 

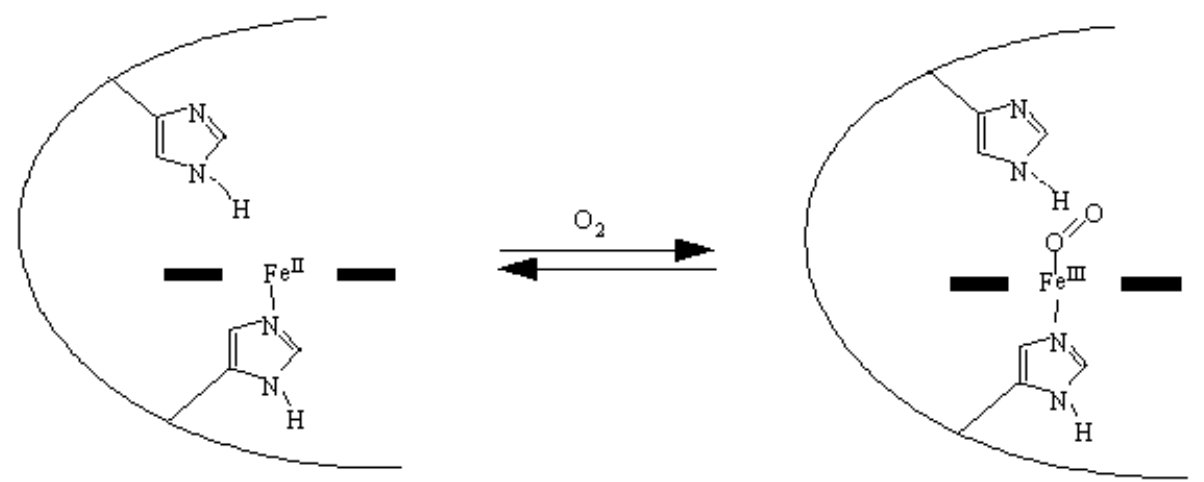

Figure 2.3.2: Oxygen Binding in Myoglobin ${ }^{30}$.

As in cytochrome $\mathrm{c}$, the porphyrin is held near the molecular surface and thus the iron center is accessible for oxygen binding and release. But unlike the cytochromes, the globin protein is not covalently linked to the porphyrin. The prosthetic heme is secured by a series of weak interactions (hydrogen bonding, non-polar, van der Waals) ${ }^{32}$. Figure 2.3.3 shows the position of the heme unit at the surface of the molecule in myoglobin. 


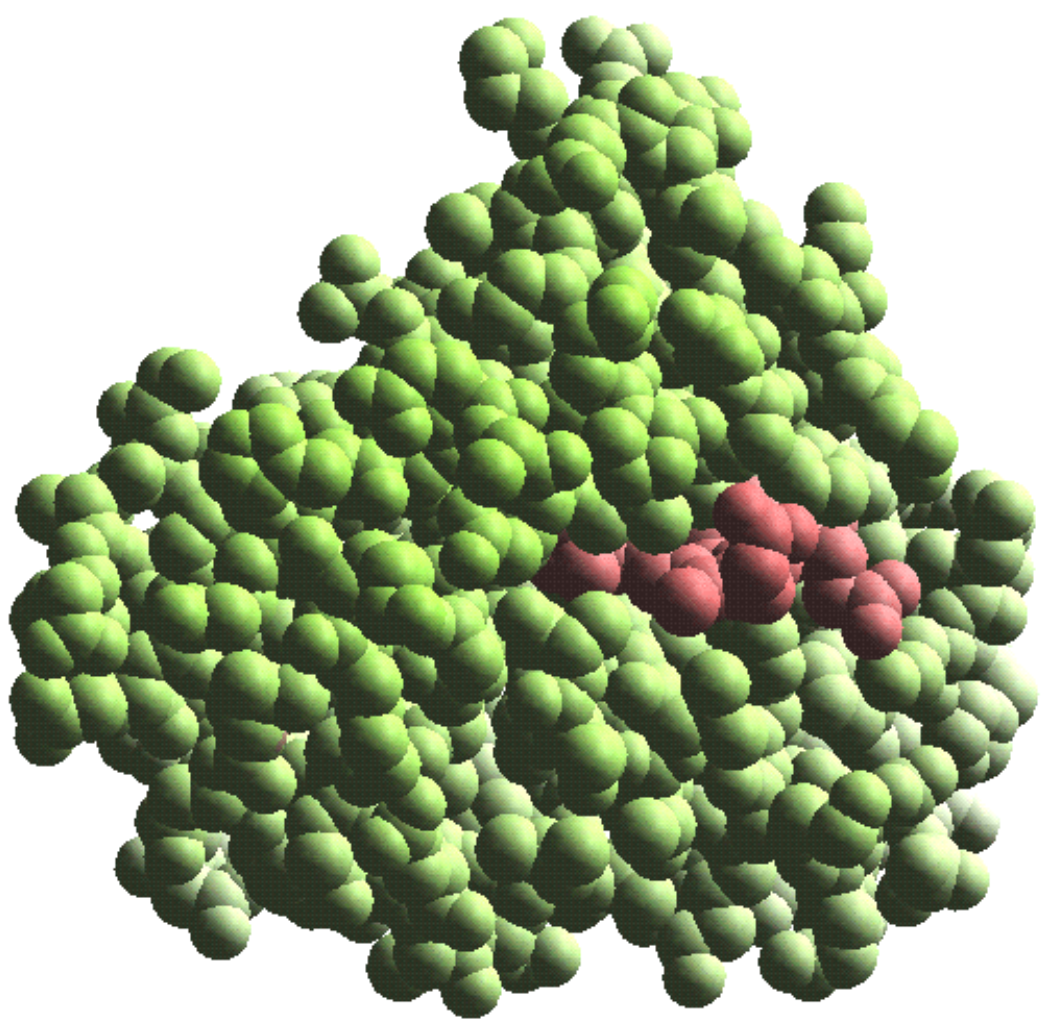

Figure 2.3.3: Heme Unit (red) at the surface of Myoglobin ${ }^{30}$ 


\subsection{Electrospray Ionization Mass Spectrometry for Protein Analysis}

The very nature of proteins and other biopolymers makes them difficult to introduce to the gas phase as intact molecular ions for mass spectometric analysis. Proteins are thermally labile, decomposing upon heating as would be necessary to accomplish thermal vaporization such as in Gas Chromatography. The large size of proteins and the presence of polar functional groups make them highly non-volatile, and thus difficult to introduce to the gas phase. Therefore the process of converting biomolecules in the condensed phase to charged species in the gas phase without causing fragmentation has long been the pursuit of those involved in instrument design.

A successful early approach to the problem was Plasma Desorption Ionization, developed by Mc Farlane and others in the 1960's ${ }^{33}$. Here the decay of ${ }^{252} \mathrm{Cf}$ produced 75 and $100 \mathrm{MeV}$ Strontium and Barium nuclei, which subsequently struck a nitrocellulose target upon which the analyte resides. The energy imparted to the target molecules in collision resulted in both vaporization and ionization of the analyte. Once in the gas phase, the molecules were analyzed by Time-of Flight Mass Spectrometry. While this approach was successful in delivering large biomolecules to the gas phase, the random nature of the decay process made interpretation difficult.

The next significant development in biological mass spectrometry was the advent of the Fast Atom Bombardment ion source by Barber in the 1970's ${ }^{34}$. In this technique, the analyte material of interest in a low volatility matrix is placed in a sample holder. A beam of atoms (typically xenon of argon) is impinged upon the glycerol target, the result of which is the sputtering of material from the matrix into the gas phase above the sample holder. The material may be ionized within the matrix as is determined by the solution chemistry, or may be ionized 
by a number of processes as a result of the energetic nature of the sputtering process. The ions are then focused by a series of lenses into a suitable mass spectrometer.

FAB marked the beginning of true biological mass spectrometry by providing a continuous, non-radioactive source for the analysis of biopolymers. The glycerol matrix served to absorb energy, reducing sample degradation. It afforded good sensitivity and analytes of a broad range of molecular weights could be analyzed by this technique. However it suffered from poor sample-to-sample reproducibility, and because the source operated at high-pressure it was difficult to interface to mass spectrometers requiring high vacuum.

The advent of electrospray ionization by Fenn and others in $1989^{5}$ created a revolution in the analysis of proteins. The ability of this method to deliver large, thermally labile biomolecules to the gas phase intact made possible the analysis of proteins that were formerly experimentally inaccessible. Since its development electrospray has become the ionization method of choice for the analysis of proteins ${ }^{6}$, as well as other biopolymers such as oligosaccharides ${ }^{7}$ and oligonucleotides $^{8}$ that have historically been difficult to analyze. To date, electrospray ionization has been employed in the study of many aspects of protein structure, including the determination of the primary sequence ${ }^{9}$ protein conformational analysis ${ }^{10}$, and in determining the oxidation states of metals in metalloproteins ${ }^{12}$. A detailed explanation of the mechanism of electrospray, as well as a description of the instrumentation used in the studies outlined in the following chapters may be found in Section 1 of this document. 


\subsection{Protein Conformations}

The shape of a protein, that is the overall conformation of the structure, is determined by all of the elements of structural hierarchy discussed in the previous section. However within each overall conformation, there are many local conformations that contribute to the overall shape of the protein.

Any polymer, including biopolymers such as proteins, possess a local conformation ${ }^{27}$ related to the orientation of its constituent monomers. Most peptides are found in a trans configuration, that is successive alpha carbons are on opposite sides of the peptide bond between them, as steric hinderance forbids the adoption of the cis configuration. An alpha helix may also be considered a local conformation, as it refers to only a portion of the entire protein. Elements of tertiary structure also create local conformations, as in the aggregation of helices to form domains within a polypeptide unit. The relationship in space of one polypeptide subunit to another, the quaternary level of protein structure, also contributes to the overall protein conformation.

All conformational levels may be significant with respect to the activity of the protein in a biological system. For instance, the presence of the D-isomer of glycine in HIV-1 protease conveys to the protein activity toward amino acids of the D-configuration, and is inactive against L-configuration substrates ${ }^{27}$. The orientation of myoglobin and hemoglobin residues into alpha helices (secondary structure), and the grouping of collections of such individual helices (tertiary structure) into a specific conformation forms a globin fold. The globin fold serves to anchor the prosthetic heme group that is the operative part of the molecule in oxygen transport and storage. The conformation of the fold creates a hydrophobic pocket wherein the heme group resides, thus protecting the "machinery" of the molecule from damage via exposure to the surrounding 
environment. Therefore the secondary and tertiary elements of structure together create a conformation that performs a specific function within the molecule. Also, the substitution of valine, a hydrophobic amino acid residue, for glutamine, a charged, hydrophilic residue, creates a hydrophobic patch on the surface of the molecule. This nonpolar region fits a nonpolar pocket on the deoxygenated form of another molecule of hemoglobin, causing red blood cells to stick together, impeding their ability to deliver oxygen to the body's tissues. This condition, known as Sickle-cell anemia, results from a single-nucleotide polymorphism that causes the substitution of valine for glutamine in the primary sequence of the beta chains ${ }^{27}$. From these examples it can readily be seen how the aspects of structure, even a single substitution at the primary level, can result in a conformational anomaly that hinders the function of the entire molecule. This also serves to illustrate the point that protein conformations, whether they are local conformations within one of many helices, or the overall shape of the molecule, can have much to say with regard to the proper function of the protein in a biological system.

\subsection{Protein Conformational Analysis by Electrospray Mass Spectrometry}

The analysis of protein conformations by ESI began in earnest in the early 1990's, as Loo ${ }^{35}$, Chowdhury ${ }^{10}$, and others observed that subjecting the protein to differing solvent conditions in solution resulted in changes to the observed Charge State Distribution (CSD) of masses observed. Unlike small molecules, proteins may accept many positive charges, and subtle variations in the shape of the molecules in solution make it possible for different molecules to adopt different charge states in solution. The number of protons associated with the structure is associated with the number of basic amino acid structures that constitute the protein. Thus the 
ESI spectrum of a protein is not comprised simply of one mass peak, but of a group of mass peaks, each corresponding to the protein and a specific number of associated protons. Thus a conformation as observed by ESI-MS is characterized not by the observation of one charge state, but a collection of charge states compromising a CSD. Figure 2.6.1 shows the CSD of cytochrome $\mathrm{c}$ in deionized water. Analysis of the spectrum reveals that the most abundant charge state is the $9+$ state (nine associated protons), but that the distribution also includes the protein with $11,10,8$, and 7 associated protons in the CSD. 


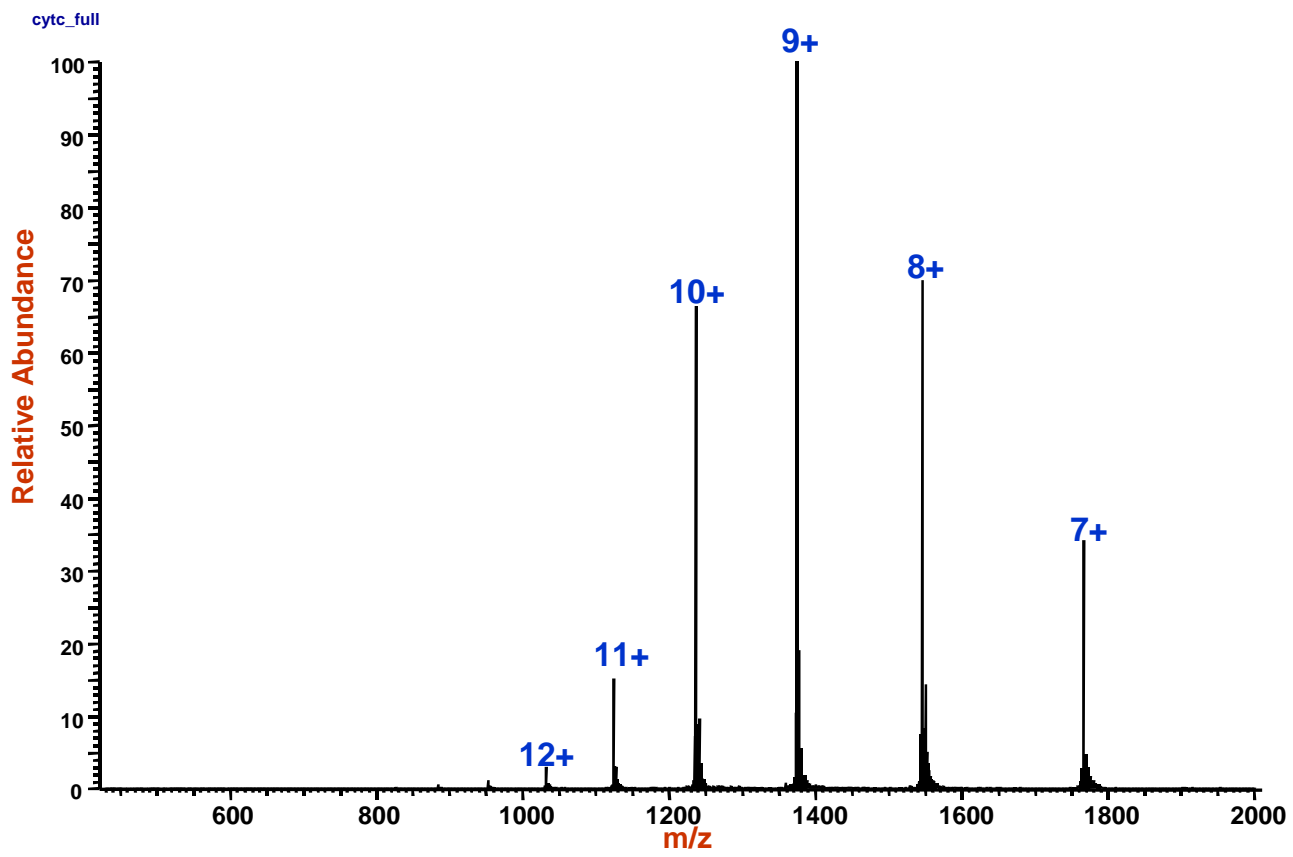

Figure 2.6.1: Charge State Distribution of Cytochrome $\mathrm{c}$ in deionized water.

Loo observed that the presence of organic solvents in solution with the protein caused significant changes in the charge state distributions of lysozyme and ubiquitin. This change in the distribution was proposed to be attributable to the disruption of hydrogen bonds that maintain the tertiary structure of the protein. This disruption induces a conformational change that exposes basic residues in the protein that are hindered in the native state to the solvent. These formerly neutral sites are then protonated, resulting in a change in the CSD of the protein to higher charge states. Therefore the CSD is not merely reflective of the total number of basic residues in the protein, but an indication of the number of these basic sites that are accessible to the solvent. Similarly, Chowdhury ${ }^{12}$ observed changes in the CSD by altering the $\mathrm{pH}$ of solutions containing 
cytochrome c. The shifts in the CSD were attributed to a conformational change that results from the change in solution $\mathrm{pH}$, altering the tertiary structure of the protein and exposing basic sites that are hindered in the native state to the solvent for protonation. Therefore both groups proposed that conformational changes in the solution phase could be monitored by ESI-MS.

Konermann developed a more elegant method for the study of protein folding by ESI-MS in 1997. Instead of inducing conformational changes by altering the $\mathrm{pH}$ of individual protein solutions and monitoring changes by analyzing a series of solutions in a range of $\mathrm{pH}$ values as in previous work, folding and unfolding was studied in real time by a technique referred to as timeresolved ESI. In this method a solution of protein and a $\mathrm{pH}$ modifier are placed in separate syringes, and are delivered by syringe pump to a mixing tee, where the two solutions may combine. The tee is connected to the ESI source by a glass capillary of known length. By altering the length of this "reaction capillary", the time of the reaction can be controlled. Spectra are acquired at various capillary lengths that relate to specific reaction times, and in this fashion, folding and unfolding kinetics may be studied. And by altering the flow rates of the $\mathrm{pH}$ modifier and the protein measurements can be made at different $\mathrm{pH}$ values. Using this technique, the kinetics of myoglobin unfolding ${ }^{36} 37$ and cytochrome c refolding ${ }^{38}$ after denaturation were studied.

Another approach to the study of protein conformations by ESI-MS was developed by the research groups of $\mathrm{Chait}^{50}$ and $\mathrm{Smith}^{39}$ by the use of hydrogen-deuterium (H-D) labeling of proteins. It is known from NMR experiments that proteins in tightly folded conformations have a lower number of hydrogens that undergo exchange reactions with the solvent. This is because these hydogens are located in the hydrophobic core of the molecule where they are inaccessible to solvent, are involved in hydrogen bonding, or are associated with salt bridges. Upon exposure 
to denaturing conditions that results in protein unfolding, the hydrophobic core of the protein may be exposed, making available formerly inaccessible basic sites for labeling. These groups also observed that in the presence of denaturing conditions, proteins were observed at higher charge states. Additionally, a significantly higher number of sites were labeled in the denatured forms as compared to the native state. Thus not only did the charge state increase as a result of the unfolding, but the overall mass of the protein increased by an amount corresponding to the number of basic sites made available by the unfolding process. These results are consistent with the assertion that higher charge states represent more unfolded forms of a protein, and that conformations generated in the solution environment are preserved after transfer to the gas phase by the process of electrospray.

More recently, similar techniques have been used to determine which specific sites along the protein are accessible to the solvent for exchange. Resing and others ${ }^{40}$ developed a technique for defining labeled sites by conducting a labeling procedure, then cooling the reaction vessel to prevent back-exchange. By digesting the labeled protein with trypsin, and sequencing the resultant peptides by CID, the actual sites of deuterium incorporation were determined. .

Using a similar approach, Eyles was able to determine which amide hydrogens were labeled in cellular retinoic acid binding protein (CRABP I) ${ }^{41}$. This research took advantage of the fact that amide hydrogens exhibit a much lower rate of exchange than do the more labile side-chain hydrogens. After fully labeling the test protein, the solution containing the labeled CRABP I was diluted with a mixture of acetic acid and water to encourage back-exchange, and the solution was immediately infused into an ESI-equipped Fourier Transform Ion Cyclotron Resonance mass spectrometer. Spectra were then recorded at times post-mixing of 0-20 minutes. By performing CID at specific times, the masses of fragment ions could be known. And because 
of the excellent resolution afforded by ICR systems, the specific sequences corresponding to the observed masses could be determined. Also, because the CID spectra were collected repeatedly at particular time points after the induction of back-exchange, information on the rates of exchange exhibited by specific sites could be gathered. Their results on exchange rates correlate well with complementary data obtained by NMR, which supports the assertion that solutionphase structure is preserved in the gas phase.

Does the mass spectrum of a protein reflect the solution phase conformation? Do conformational changes take place during the electrospray process? How well do gas-phase measurements of proteins correlate with solution-phase measurements? Is there a direct correlation between the solution environment and what is seen in the mass spectrum? These questions and others strike at the very heart of conformational analysis by mass spectrometry, and the answers will determine the validity of this technique. Though widely applied, until these questions are answered unambiguously ESI-MS will not be universally accepted as a method to study protein conformations. The following chapters will seek to provide some insight into the correlation between solution-phase chemistry and gas-phase measurements, and to provide partial, if not complete, answers to the aforementioned questions with regard to the validity of ESI-MS as a tool for protein conformational analysis. 


\section{Chapter 3: Influence of Ion Source Parameters on the Charge-State Distributions of Cytochrome C}

\subsection{Introduction}

In recent years, electrospray ionization mass spectrometry ${ }^{6}$ has emerged as a powerful tool in protein analysis. Owing to the non-destructive nature of the ionization, and the ability of the technique to present large, thermally labile molecules to the gas phase intact, electrospray has supplanted other methods to become the most widely applied strategy for the analysis of proteins. In addition to the "soft" nature of the ionization process that occurs in electrospray ionization ${ }^{24}$, the method typically produces multiply charged species. In mass spectrometric methods, ions are observed at characteristic mass/charge $(\mathrm{m} / \mathrm{z})$ values. A significant limitation in protein analysis has been the ability of the most cost-effective mass analyzers to measure the masses of large species, as Quadrupole Mass Analyzers (QMA) and Quadrupole Ion Traps (QIT) possess upper mass limits of approximately 4000 atomic mass units. Because electrospray produces multiply, and often times highly charged ions, proteins of masses in excess of 50,000 daltons may be observed on QIT and QMA systems. Thus in addition to providing a means to introduce large, thermally labile species to the gas phase intact, electrospray ionization has expanded the effective mass range of these common mass analyzers. In interfacing an electrospray ion source to an ion trap mass spectrometer affording $\mathrm{MS}^{\mathrm{n}}$ capabilities ${ }^{18}$, the analyst has at his disposal an affordable, sensitive and reliable method of analyzing proteins. This combination has proven extremely useful in protein sequencing and identification ${ }^{6}$, and in conformational analysis. ${ }^{9,10}$ 
In this work the influence of ion source parameters (sheath gas flow rate, heated capillary temperature, capillary voltage, and tube lens voltage) on the observed charge state distribution (CSD) of cytochrome $\mathrm{c}$ is reported. A method of calculating the average observed charge (AOC) for a given spectrum is reported as well. The AOC is a useful tool for monitoring protein conformational changes by ESI-MS.

\subsection{Materials and Methods}

Bovine heart cytochrome c was purchased from Sigma (St. Louis, MO) and was used without further purification. Deionized (DI) water was obtained from an in-house purification system. Acetonitrile (HPLC Grade) was purchased from Fisher Scientific (Fair Lawn, NJ).

Standards were prepared in DI at a concentration of $1 \times 10^{-4} \mathrm{M}$. Solutions for analysis

were made by diluting the standard in DI with DI and acetonitrile to $1 \times 10^{-5} \mathrm{M}$ and $1 \times 10^{-6} \mathrm{M}$. Solutions were prepared at $0 \%, 20 \%$, and $40 \%$ acetonitrile in water at 1 and $10 \mu \mathrm{M}$ concentrations. One percent acetic acid (v/v) was added to all samples to enhance electrospray stability and sensitivity.

Spectra were acquired on a Ion Trap (Finnigan LCQ ${ }^{\circledR}$ ) Mass Spectrometer. Table 3.1.1 outlines the experimental parameters investigated in this study. Solutions were infused to the ion source at a flow rate of $3 \mu \mathrm{l} / \mathrm{min}$. for a period of one minute. The resulting scans (approximately 100) were averaged to generate a mass spectrum for the acquisition 


\begin{tabular}{|ll|}
\hline Instrument Parameter & Setpoints Evaluated \\
\hline Capillary Voltage & $-2,3$, and $8 \mathrm{~V}$ \\
\hline Heated Capillary Temperature & 150,200, and $250^{\circ} \mathrm{C}$ \\
\hline Sheath Gas Flow Rate & 20,30, and 40 units \\
\hline Auxiliary Gas Flow Rate & 5,10, and 15 units \\
\hline Tube Lens Offset Voltage & 0,30, and $60 \mathrm{~V}$ \\
\hline Sample Infusion Rate & 3,6, and $9 \mu 1 / \mathrm{min}$ \\
\hline
\end{tabular}

Table 3.2.1: Instrument Parameters and Setpoints Evaluated. 


\subsection{Experimental}

In order to evaluate conformational changes when changing parameter settings, we have found it useful to calculate a value which we refer to as the average observed charge (AOC) for a given spectrum. This is done by multiplying the intensity of each most abundant charge state peak (in $\%$ relative abundance) times the respective charge, resulting in a weighted response for the given charge state. By summing the weighted responses over all of the observed charge states and then dividing by the sum of all intensities for the most abundant mass, the AOC is obtained. We have found it useful to calculate this parameter when evaluating conformational changes, as it assigns a numerical value to a given spectrum that can be compared to other spectra obtained under different conditions. Thus, in addition to a "picture" that is the mass spectrum, we have a number that corresponds to the masses observed in the same spectrum. While this simplification ignores isotopes and shoulder peaks which when incorporated might lead to a more accurate calculation of average charge, we feel that it is a good first approximation in observing conformational changes. It affords the advantage of allowing for changes to be observed that might not be noticeable in viewing a full-scan mass spectrum.

\subsection{Results}

Capillary Voltage, Sheath Gas Flow Rate, Auxiliary Gas Flow Rate, Sample Infusion Rate No significant changes to the Charge State Distributions (CSD's) or Average Observed Charge (AOC) were observed for these parameters for any of the three solutions tested. 


\section{Heated Capillary Temperature}

Temperatures of 150,200 , and $250{ }^{\circ} \mathrm{C}$ were evaluated. While no significant changes to the CSD or AOC were observed for the sample in deionized water, a trend toward lower charges was observed with increased capillary temperature in the $20 \%$ and $40 \%$ acetonitrile samples. Figures 3.4.1 - 3.4.3 show the mass spectrum of cytochrome $\mathrm{c}$ in $20 \%$ acetonitrile at the three capillary temperatures evaluated. Figures 3.4.4 - 3.4.6 show the mass spectrum of cytochrome c in $60 \%$ acetonitrile at the three tested temperatures. 
cyto5_41 \#1-27 RT: 0.03-1.00 AV: 27 NL: 1.77E6

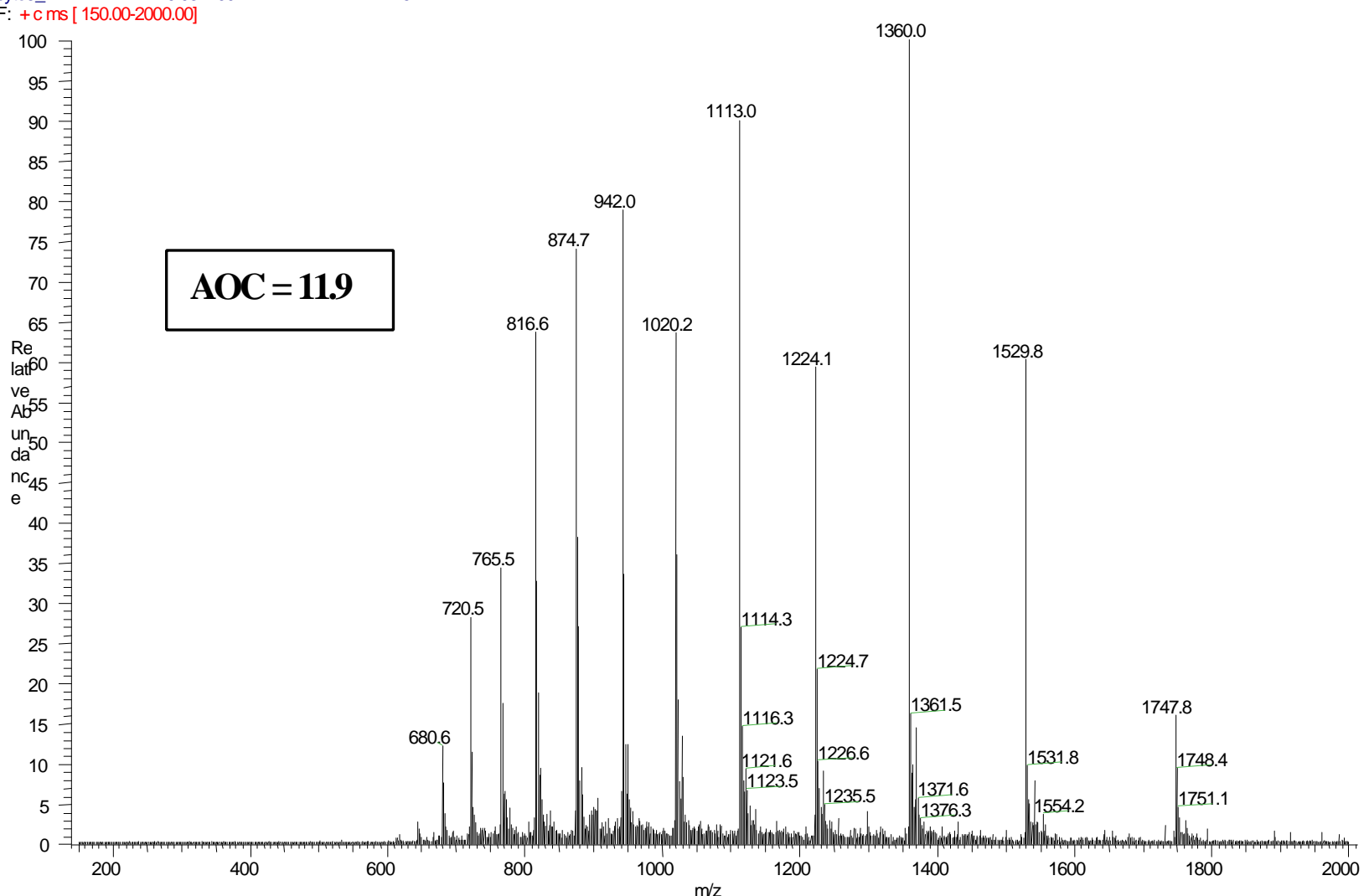

Figure 3.4.1: Mass spectrum of cytochrome $\mathrm{c}$ in $20 \%$ acetonitrile, capillary temperature $=150^{\circ} \mathrm{C}$. 


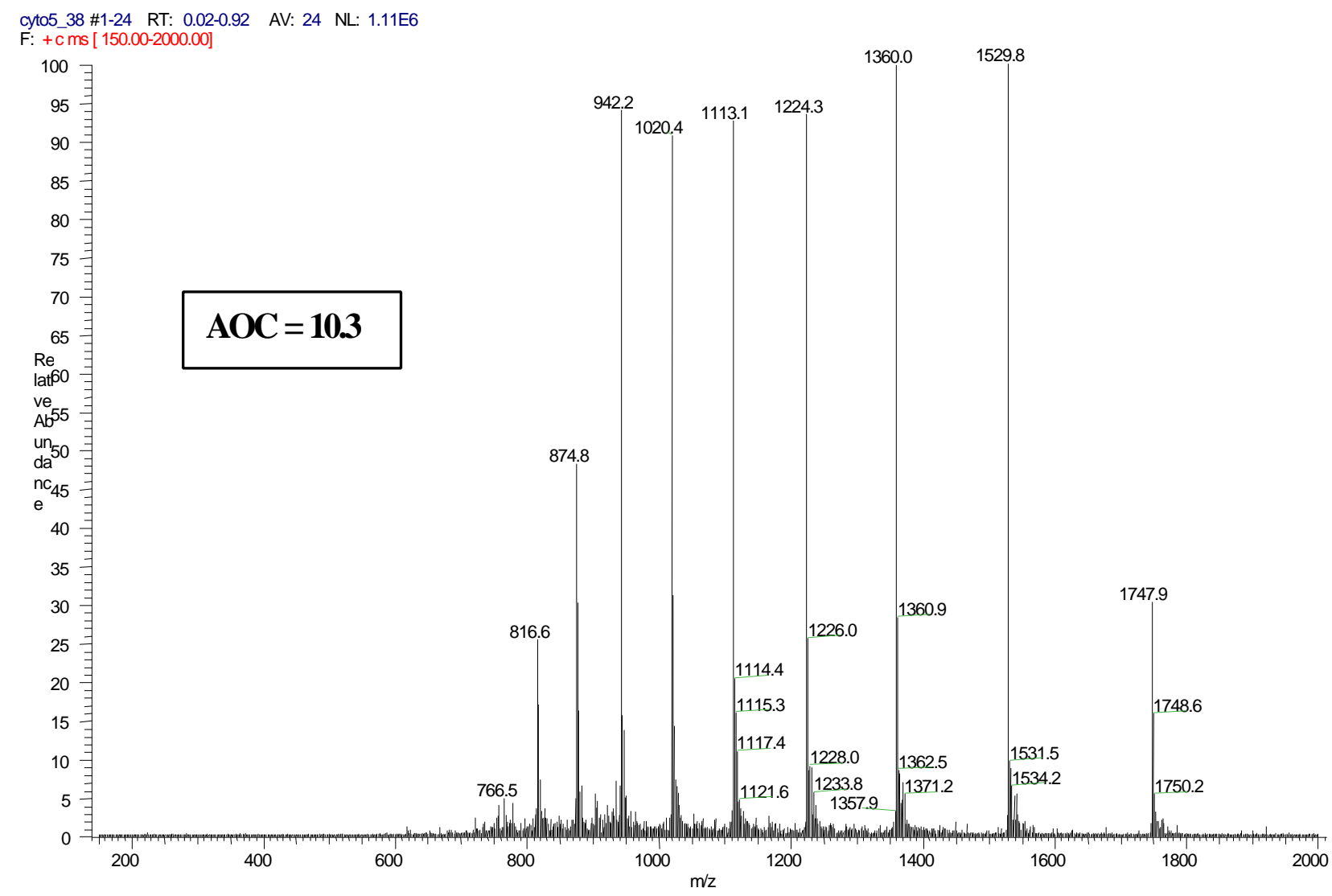

Figure 3.4.2: Mass spectrum of cytochrome $\mathrm{c}$ in $20 \%$ acetonitrile, capillary temperature $=200{ }^{\circ} \mathrm{C}$. 


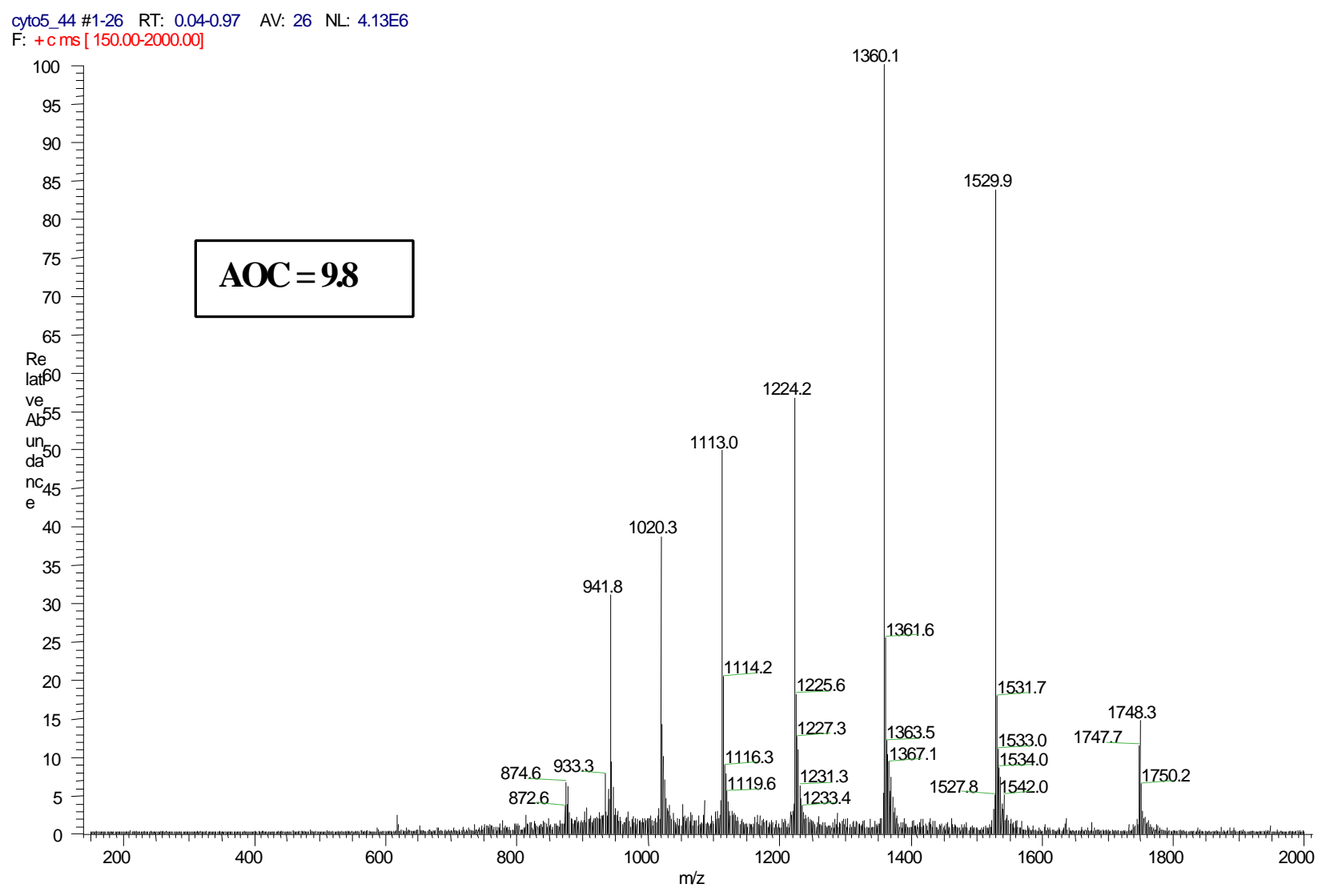

Figure 3.4.3: Mass spectrum of cytochrome $\mathrm{c}$ in $20 \%$ acetonitrile, capillary temperature $=250{ }^{\circ} \mathrm{C}$. 


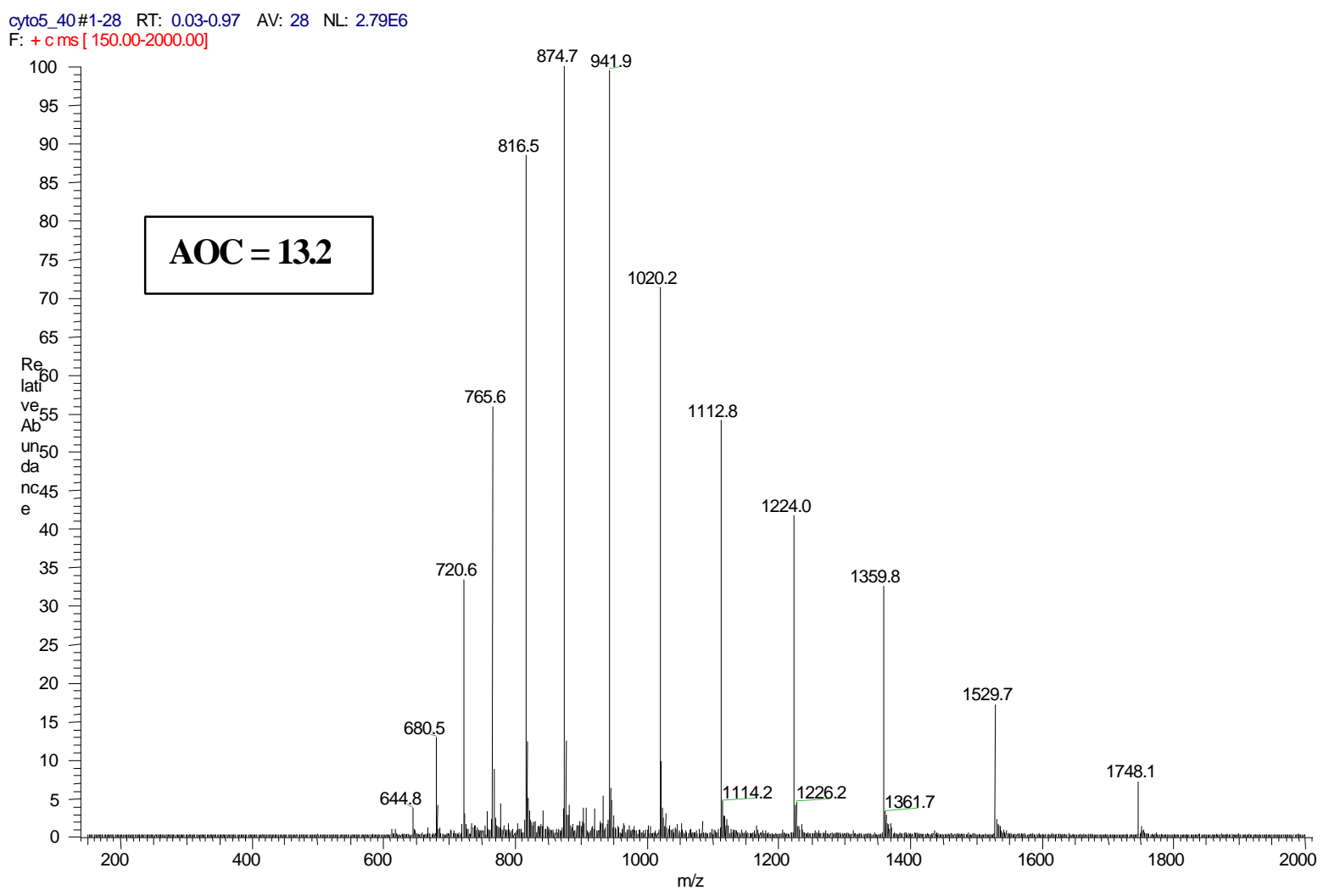

Figure 3.4.4: Mass spectrum of cytochrome $\mathrm{c}$ in $60 \%$ acetonitrile, capillary temperature $150{ }^{\circ} \mathrm{C}$. 
cyto5_39 \#1-26 RT: $0.02-0.97 \quad$ AV: $26 \quad$ NL: 1.33E6

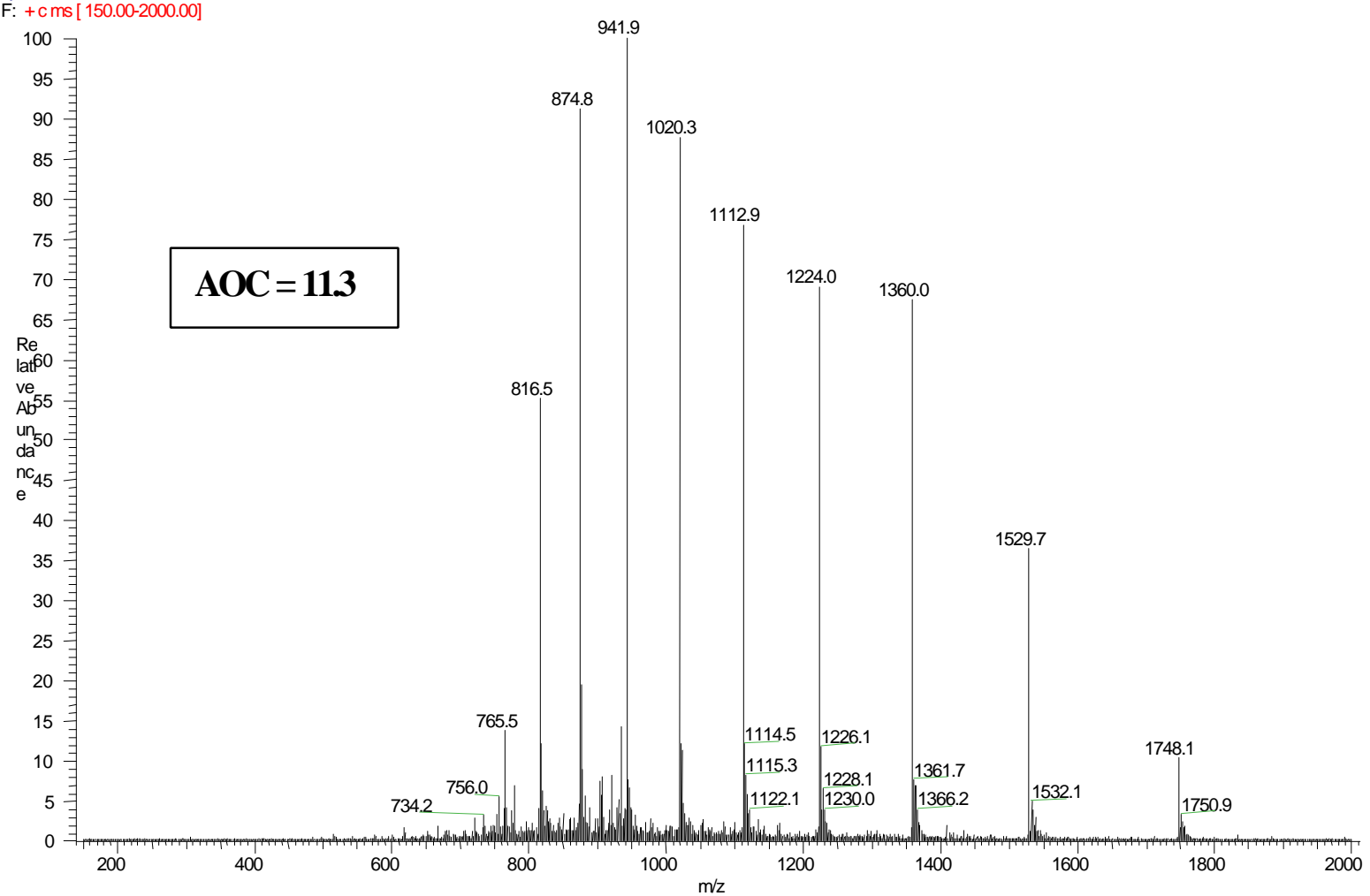

Figure 3.4.5: Mass spectrum of cytochrome $\mathrm{c}$ in $60 \%$ acetonitrile, capillary temperature $=200{ }^{\circ} \mathrm{C}$. 


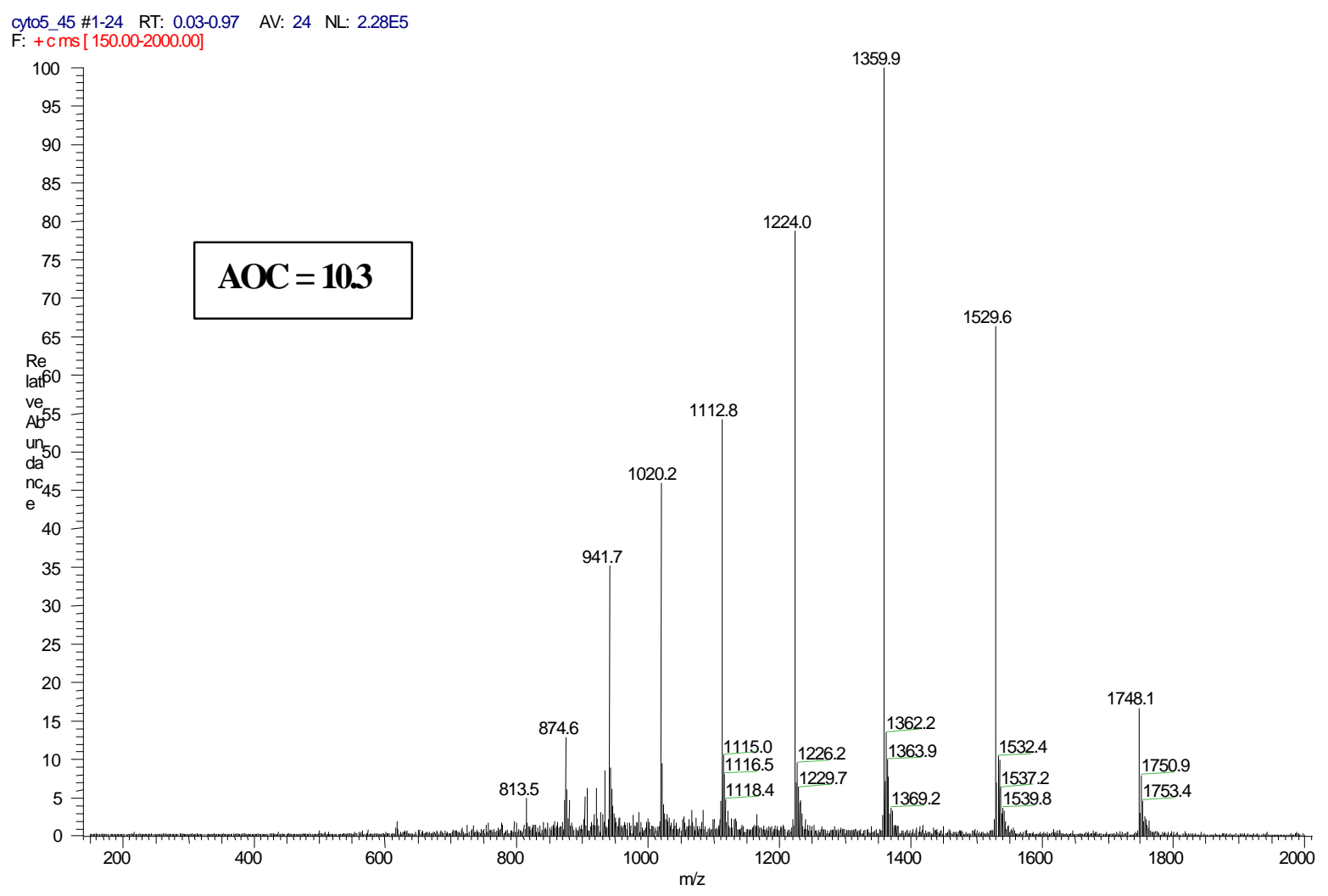

Figure 3.4.6: Mass spectrum of cytochrome $\mathrm{c}$ in $60 \%$ acetonitrile, capillary temperature $=250{ }^{\circ} \mathrm{C}$. 
Table 3.4.1 summarizes the influence of capillary temperature on the AOC for the three conformations tested, clearly indicating a trend toward lower charges (higher masses) at increased capillary temperature.

\begin{tabular}{|c|c|c|c|}
\hline & & $\mathrm{AOC}$ & \\
\hline $\begin{array}{c}\text { Capillary Temperature } \\
\left({ }^{\circ} \mathbf{C}\right)\end{array}$ & $0 \%$ Acetonitrile & $20 \%$ Acetonitrile & $60 \%$ Acetonitrile \\
\hline 150 & 8.35 & 11.92 & 13.16 \\
\hline 200 & 7.94 & 10.28 & 11.26 \\
\hline 250 & 7.93 & 9.80 & 10.30 \\
\hline
\end{tabular}

Table 3.4.1: Influence of Heated Capillary Temperature on the Average Observed Charge (AOC) for the three tested solutions. 


\section{Tube Lens Offset Voltage}

Initially tube lens voltage settings of $0,+30$, and $+60 \mathrm{~V}$ were used to evaluate the impact of this parameter on the CSD of cytochrome c. A trend toward lower charges in the CSD with increased tube lens voltage was observed when analyzing the denatured forms. No significant change was seen in the CSD for the native form in deionized water. Table 3.4.2 shows the influence of tube lens offset voltage on the AOC's for the three tested solutions.

\begin{tabular}{l|lll} 
& \multicolumn{3}{|c}{ AOC } \\
\hline Tube Lens Voltage & $0 \%$ acetonitrile & $20 \%$ acetonitrile & $60 \%$ acetonitrile \\
$\mathbf{0}$ & 8.15 & 12.08 & 14.73 \\
$\mathbf{3 0}$ & 7.90 & 10.53 & 12.70 \\
$\mathbf{6 0}$ & 8.30 & 9.57 & 8.86
\end{tabular}

Table 4.3.2: Influence of Tube Lens Voltage on the AOC's of Cytochrome c.

In order to more thoroughly evaluate this effect, a broader range of tube lens settings was examined for impact of this parameter upon the CSD. Data was acquired at tube lens settings of $-15,0,15,30,45,60,75$, and $90 \mathrm{~V}$. Upon analysis of the data by visual inspection of the spectra and calculation the AOC's, the trend toward lower charges with increased tube lens voltage was again seen for the solutions containing the denatured forms of cytochrome c. Figures 3.4.73.4.12- show the mass spectrum of cytochrome $\mathrm{c}$ in $20 \%$ and $60 \%$ acetonitrile at tube lens voltage settings of 0,30 , and $60 \mathrm{~V}$. Figure 3.4 .13 summarizes the changes to the $\mathrm{AOC}$ as a result of varying the tube lens voltage for all three tested solutions. 
cyto12_10\#1-26 RT: 0.01-0.97 AV: $26 \quad$ NL: 3.36E6

$\mathrm{F}:$ + c ms [ 150.00-2000.00]

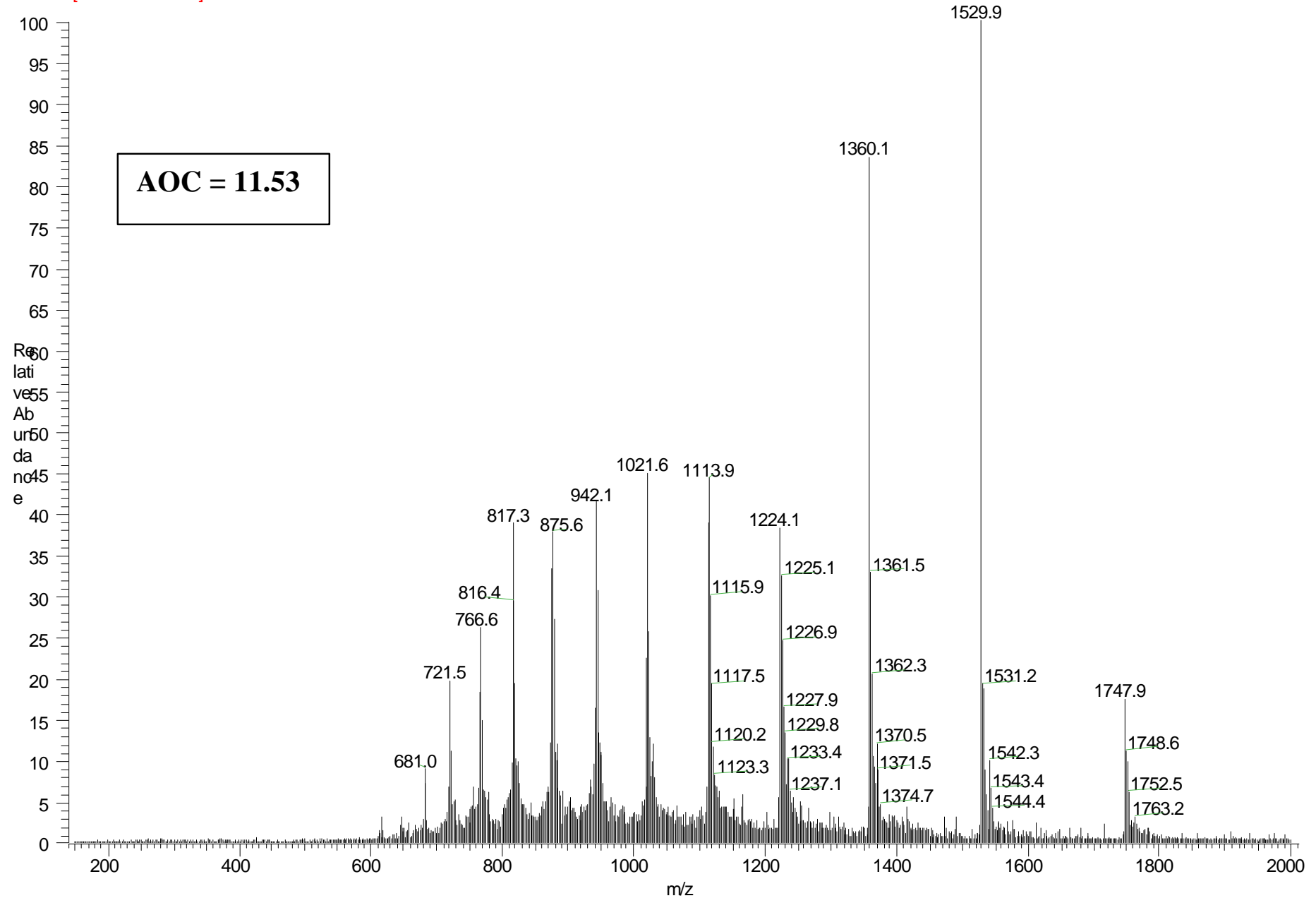

Figure 3.4.7: Mass spectrum of cytochrome $\mathrm{c}$ in $20 \%$ acetonitrile, tube lens $=0 \mathrm{~V}$. 


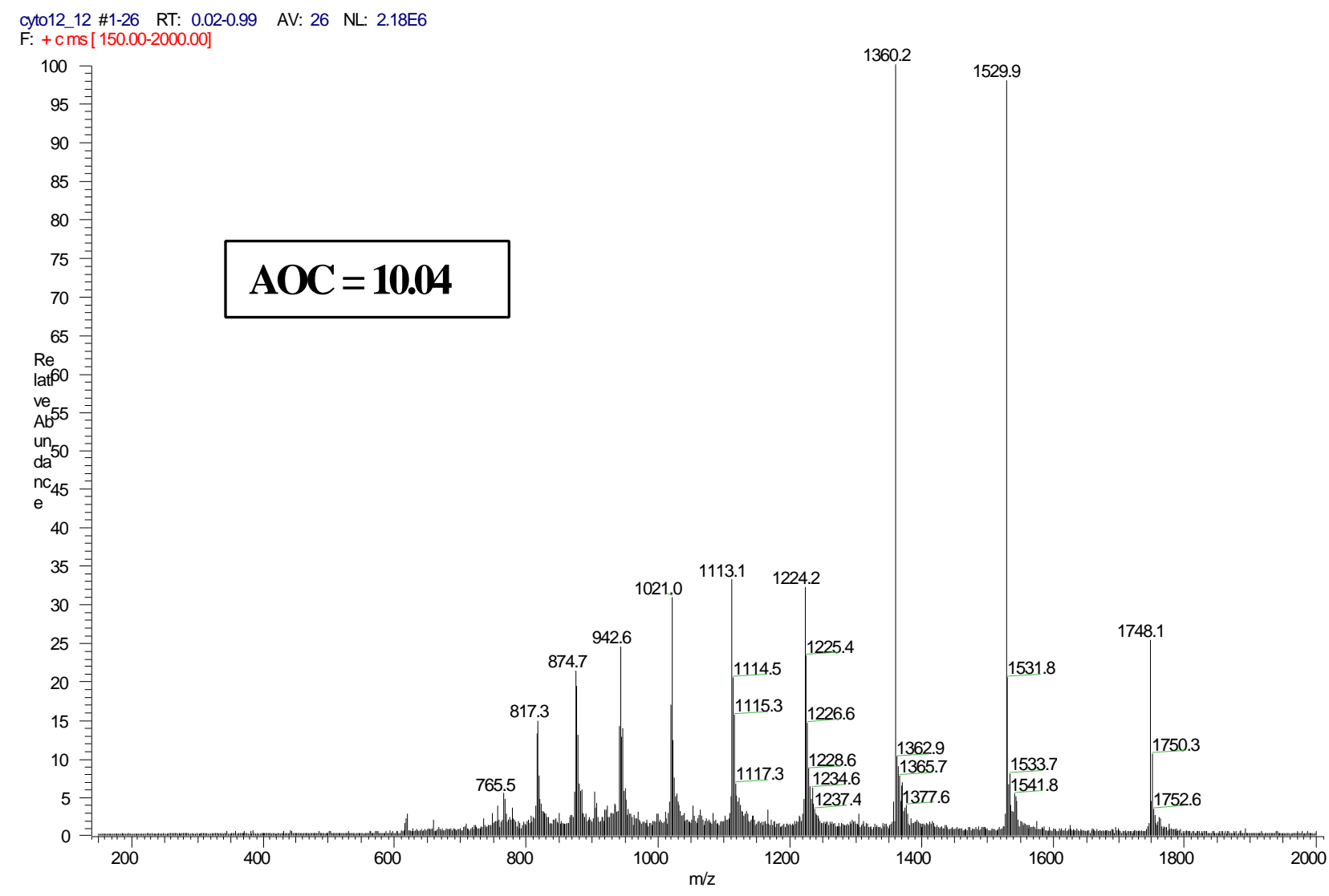

Figure 3.4.8: Mass spectrum of cytochrome $\mathrm{c}$ in $20 \%$ acetonitrile, tube lens $=30 \mathrm{~V}$. 


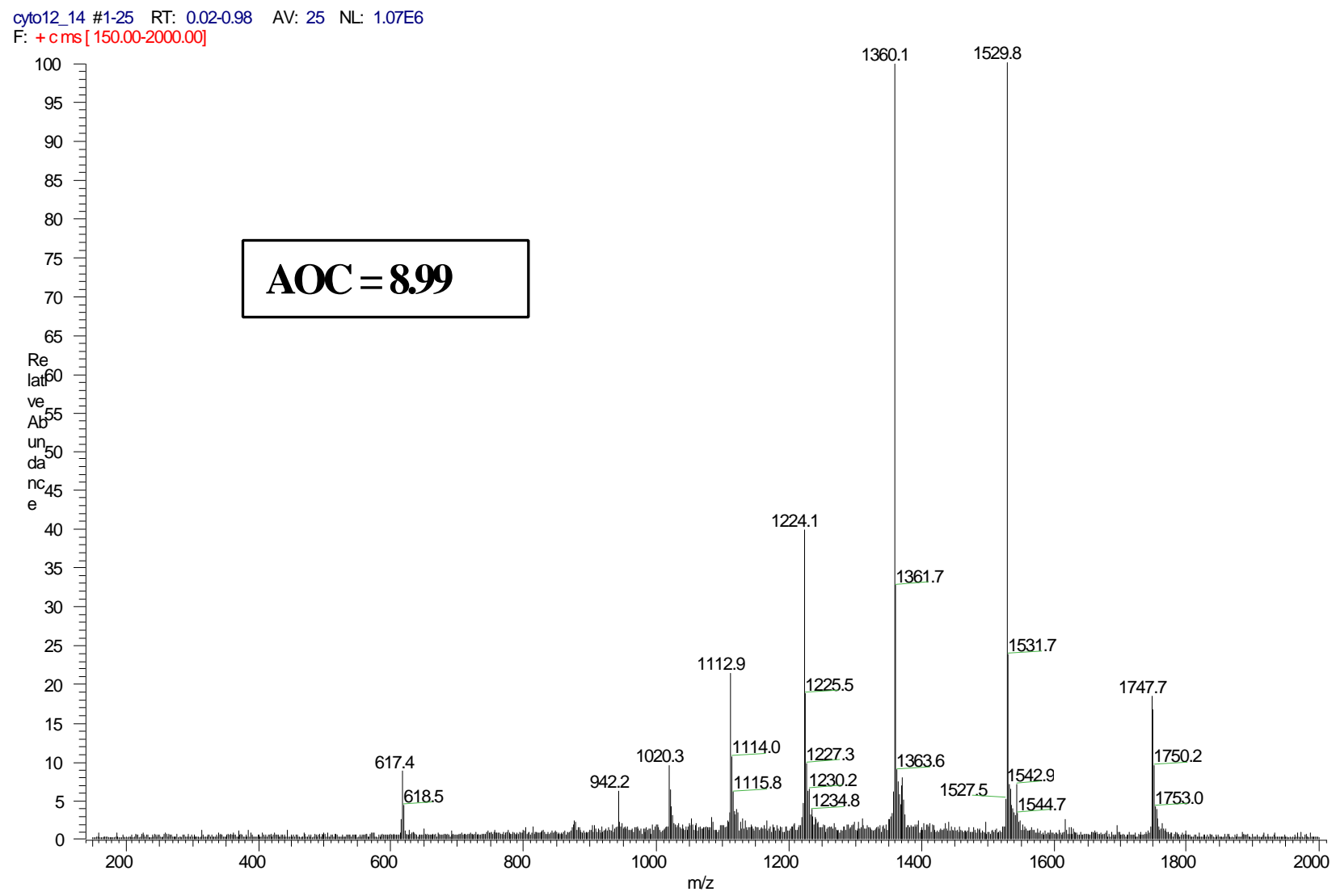

Figure 3.4.9: Mass spectrum of cytochrome $\mathrm{c}$ in $20 \%$ acetonitrile, tube lens $=60 \mathrm{~V}$. 


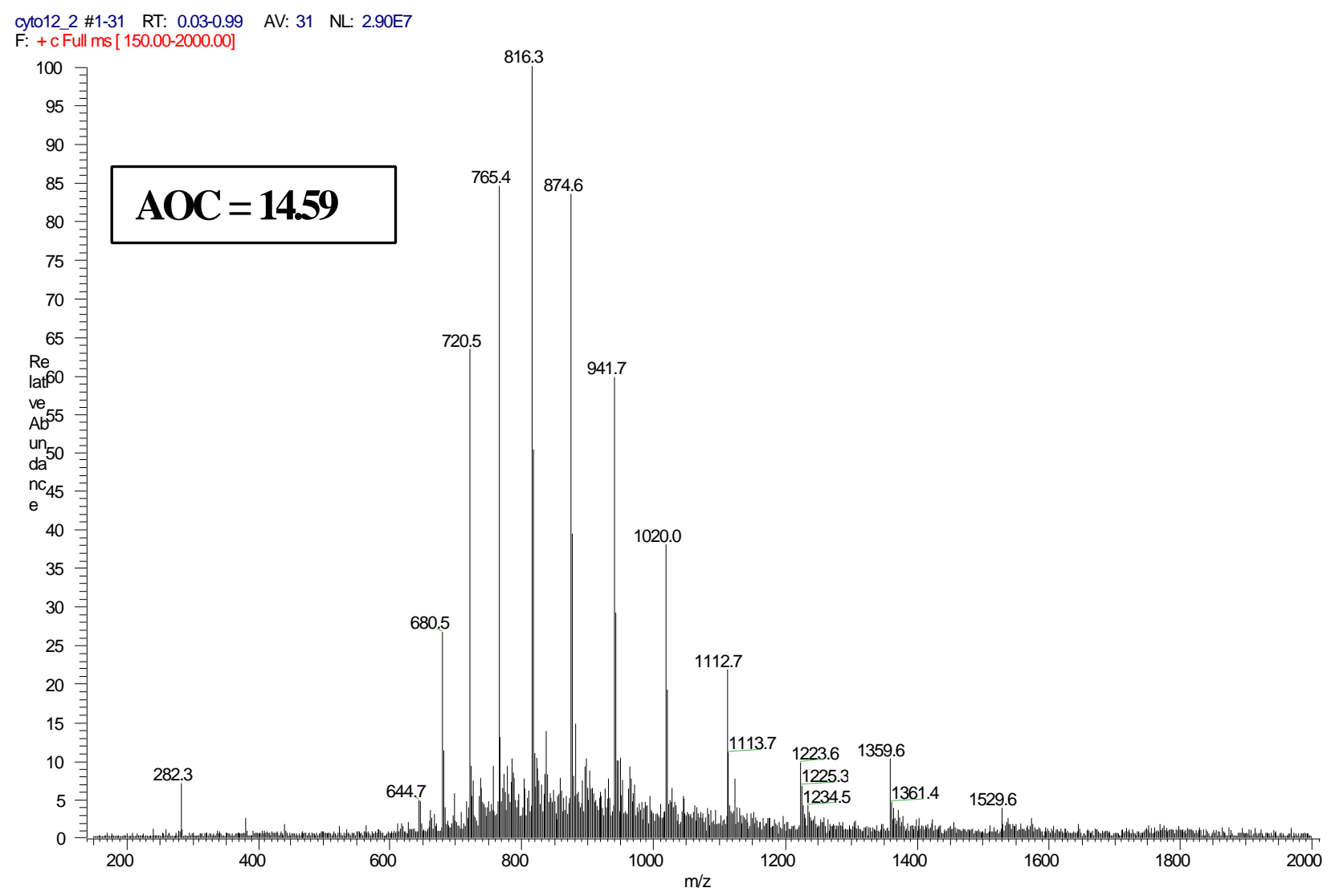

Figure 3.4.10: Mass spectrum of cytochrome $\mathrm{c}$ in $60 \%$ acetonitrile, tube lens $=0 \mathrm{~V}$. 


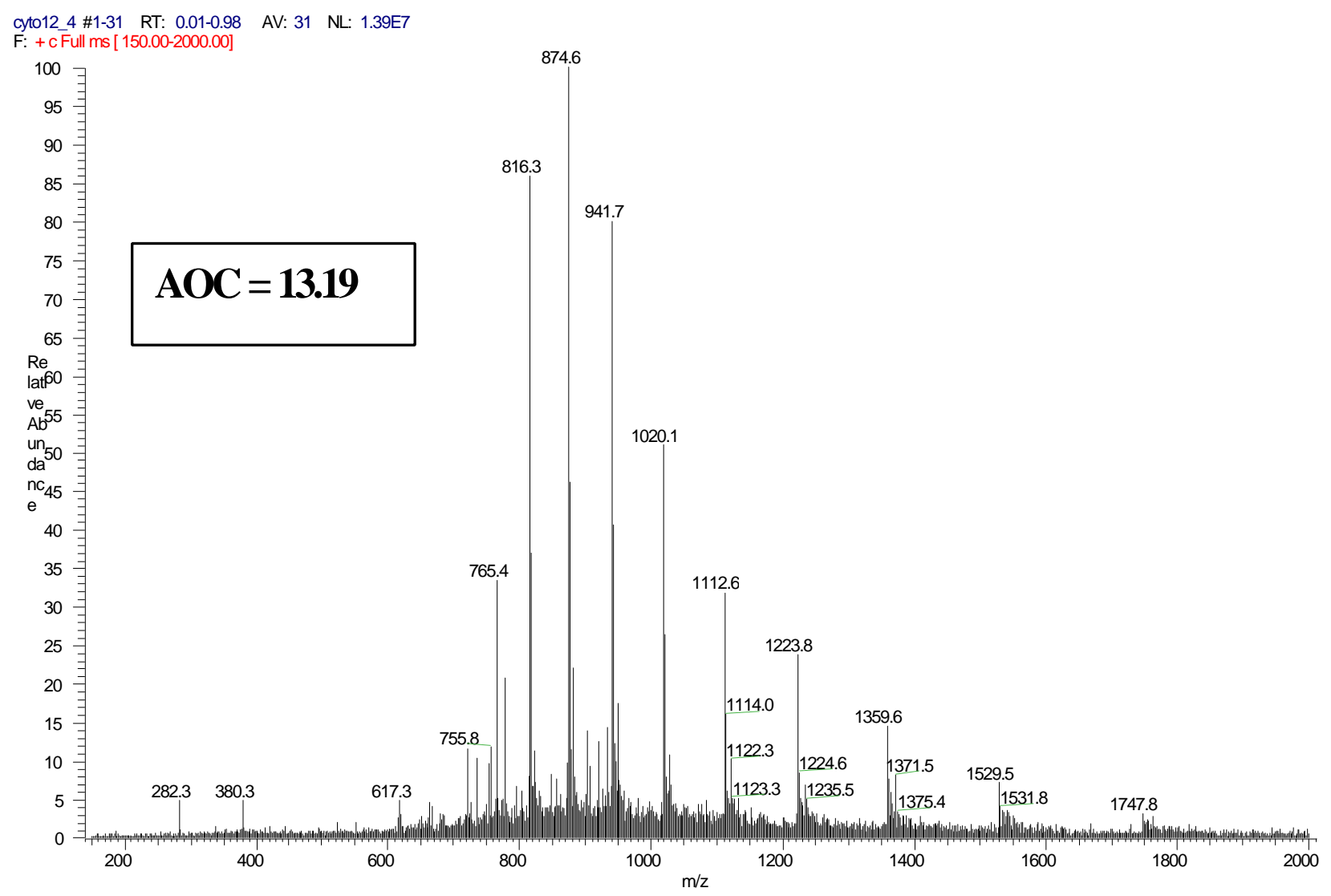

Figure 3.4.11: Mass spectrum of cytochrome $\mathrm{c}$ in $60 \%$ acetonitrile, tube lens $=30 \mathrm{~V}$. 


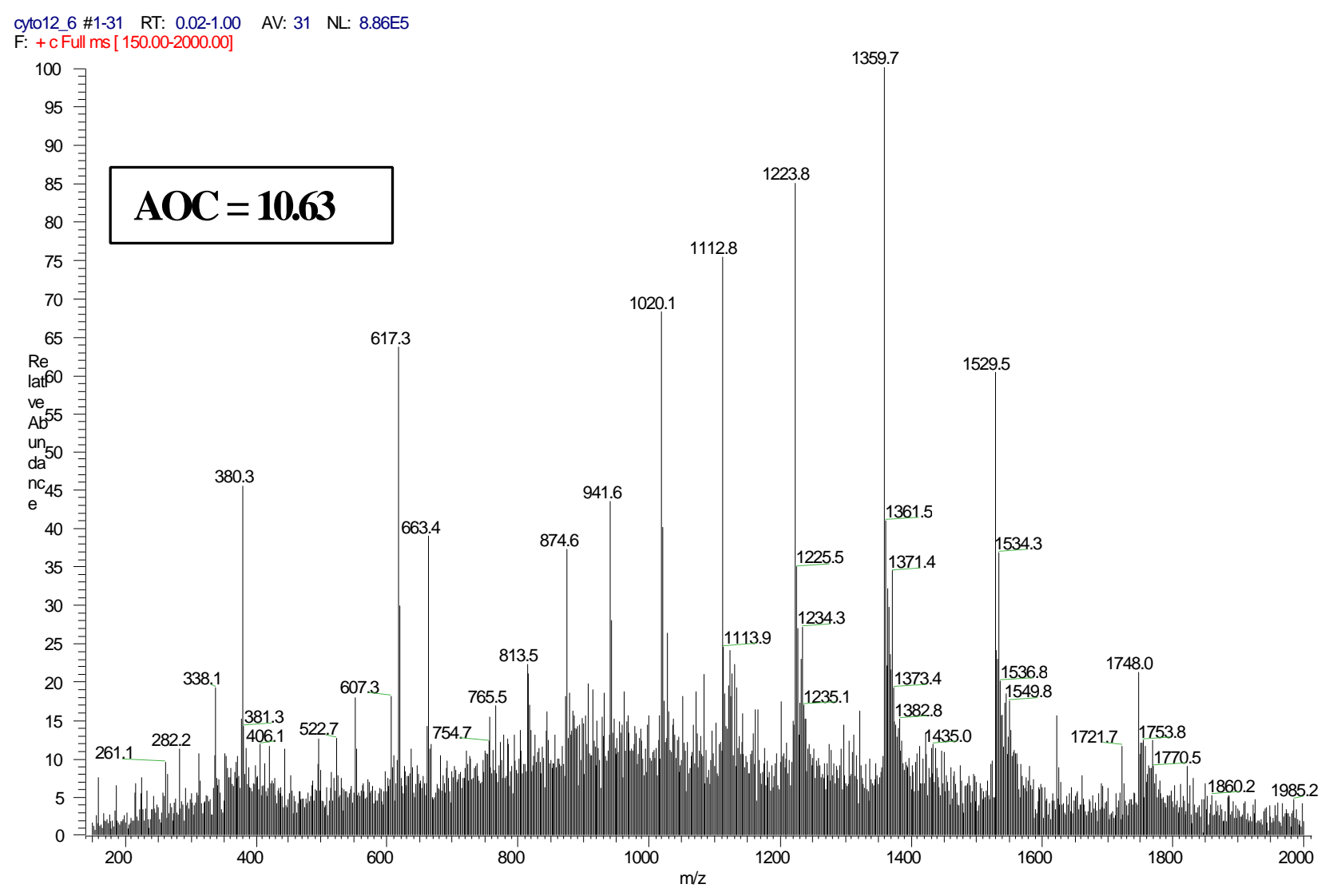

Figure 3.4.12: Mass Spectrum of cytochrome $\mathrm{c}$ in $60 \%$ acetonitrile, tube lens $=60 \mathrm{~V}$. 
Tube Lens Voltage vs. Average Observed Charge

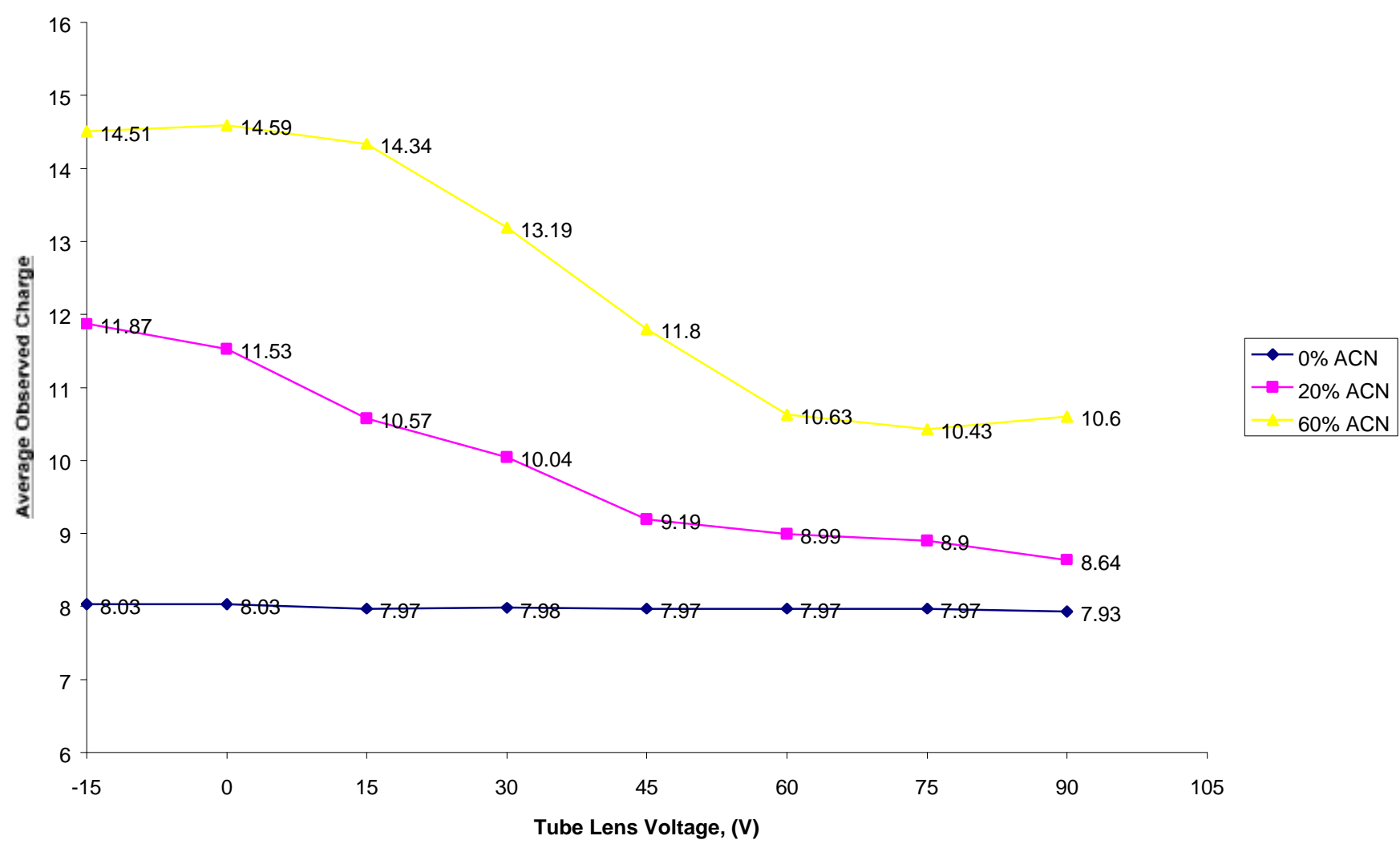

Figure 3.4.13: Average Observed Charge vs. Tube Lens Voltage for Cytochrome c in0, 20, and 60\% Acetonitrile.

While no change is seen for the protein in deionized water, a stepwise trend toward lower average charge is seen when the tube lens voltage is increased for the samples containing $20 \%$ and $60 \%$ acetonitrile. Linear regression of the data between -15 and $60 \mathrm{~V}$ for the $20 \%$ acetonitrile sample suggests a linear relationship $\left(\mathrm{R}^{2}=0.9887\right)$ between tube lens voltage and average charge over this range of voltages. A similar relationship was observed for the $60 \%$ acetonitrile sample $\left(R^{2}=0.9807\right)$. 
In order to evaluate the reproducibility of this effect, the solutions were analyzed again two days removed from the original analysis. Figure 3.4.14 shows the influence of the tube lens voltage on the average observed charge on different days. Mean percent difference for each data point was calculated to be less than $6 \%$, indicating good reproducibility for this effect.

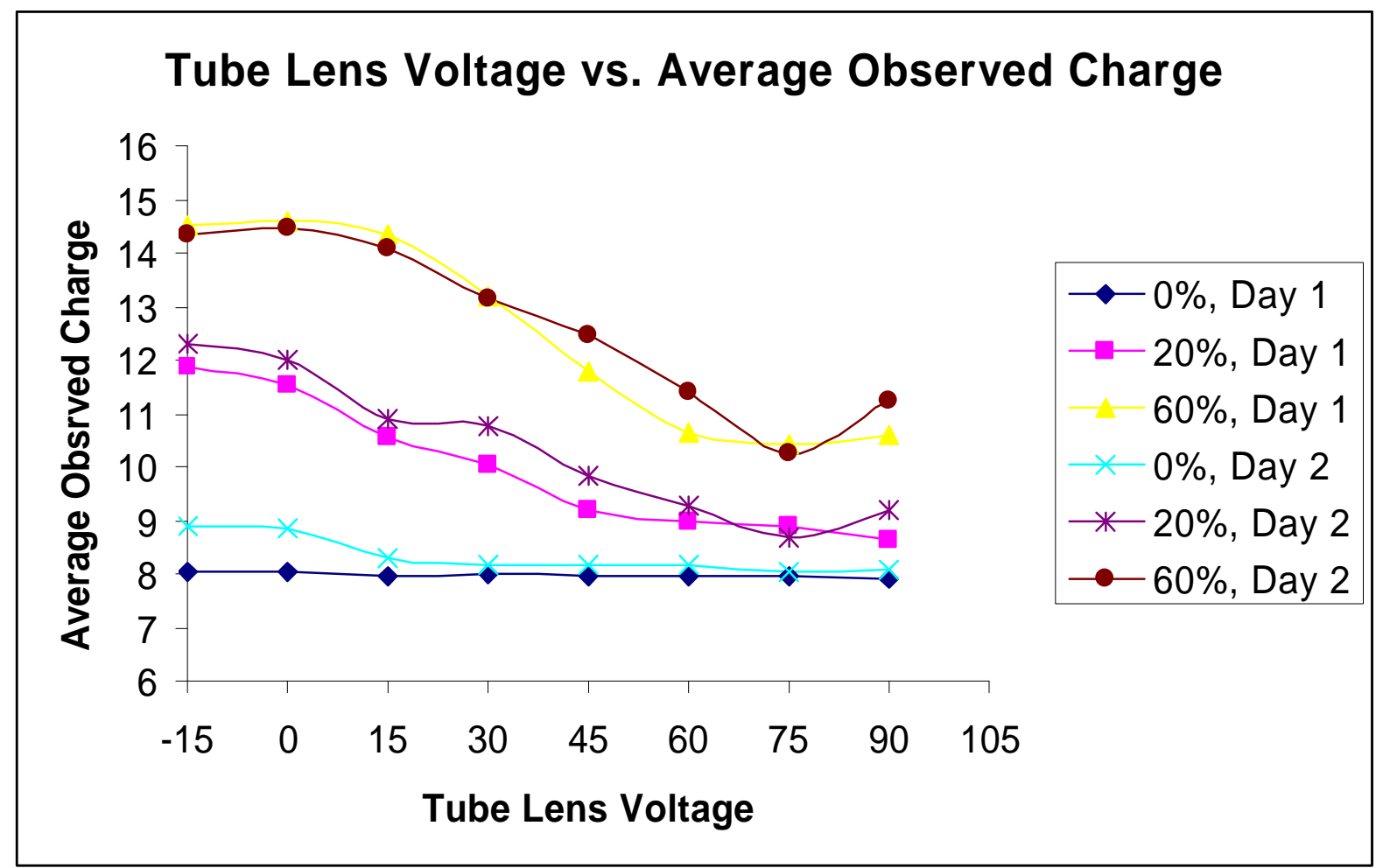

Figure 3.4.14: Interday Variability of Tube Lens Settings 


\subsection{Discussion}

The trend toward lower charge states at increased capillary temperatures is interesting, and would appear to conflict with the theories of Fenn ${ }^{42}$, who proposed that increased rates of desolvation favor higher charge states due to reduced residence time of ions in areas of low charge density. While this may be true when desolvation is accomplished by use of a curtain gas of nitrogen alone, it does not appear to hold true for electrospray sources employing a heated capillary tube to complete the desolvation process. I propose that this effect is not due to a shift in the ions that are formed, but a change in which ions are detected. Loo $^{43}$, Busman ${ }^{44}$, and others have reported increased reactivity with increasing charge state for a number of proteins. The increase in temperature may impart to the molecules sufficient energy to make possible new reaction pathways that are not accessible at lower temperatures. The more reactive higher charge ions, having been modified by some reaction (oxidation, reduction, etc.) would disappear from the spectrum, or shift in mass. This would bias the spectra at higher temperatures toward the lessreactive, lower charge states.

The impact of the tube lens voltage upon the mass distribution was significant, step-wise with voltage increments, linear, and reproducible. While some losses in sensitivity were noted in the $60-90 \mathrm{~V}$ range, good ion throughput was maintained in moving from $0-45 \mathrm{~V}$, with a corresponding change from an $\mathrm{AOC}$ of 14.59 at $0 \mathrm{~V}$ to 11.80 at $45 \mathrm{~V}$ for the $60 \%$ acetonitrile sample. This represents a change in the average charge of -2.8 over the regime. The $20 \%$ acetonitrile sample demonstrated a change of -2.25 in the AOC over the same range of voltages. Therefore, the charge state distribution may be influenced to the extent of $2-3$ charges while preserving reasonable sensitivity by adjusting the tube lens offset voltage setting. 
Although little is found in the literature with regard to this effect, recent work by Ashton $^{45}$ and others may shed some light on the situation. Their work focused on the impact of varying the skimmer voltage on protein mass distributions, reporting that an increase in skimmer potential favors lower charge states. The authors attribute this effect to an increased rate of proton transfer in the skimmer region. On Finnigan $\mathrm{LCQ}{ }^{\circledR}$ systems, the skimmer is fixed at ground potential and the tube lens, which resides just behind the skimmer, is held at a userdefined potential. Thus because the tube lens is the first field the ions experience on their way to the ion trap, an increase in the tube lens voltage may have a similar effect as that seen by Ashton.

In the positive ion mode, a positive voltage applied to the tube lens should have a repulsive effect on the ions, reducing their velocity. This would translate to increased dwell time in the skimmer-tube lens region, allowing more time for ion-molecule interactions to occur. Because the higher charge states are more reactive, a greater number of these species might participate in such reactions. This would bias the spectrum toward lower charge states, as the intensity of lower charge states would be compromised to a lesser extent than higher ones by reactions in the tube lens-skimmer region. It may also be that the reduced velocity experienced by the ions due to the repulsive force applied to the tube lens may slow the ions to the extent that injection into the ion trap is inefficient. The RF octapole ion guides may not, given the reduced velocity of these ions, at the RF settings in use be able to capture the highly charged species and efficiently guide them into the ion trap.

It seems then that the observed shift in average charge is a change in the ions that are observed, not in those created. The voltage applied to the tube lens is very small with respect to the $4 \mathrm{kV}$ applied to that capillary tip where ionization in begun. It does not seem reasonable to think that a change of 50 or 60 volts to the tube lens, which is some distance removed from the 
capillary tip held at $4 \mathrm{kV}$, could have such a dramatic effect upon the ions formed. The distance between them is too great, and the magnitude of the change too small, to attribute the observed changes in the AOC, as a result of changing the tube lens setting, to a change in which ions are formed. It is much more reasonable to suggest that the observed changes are attributable to chemical processes in the tube lens-skimmer region or changes to ion kinetics that alter the distribution of ions observed.

\subsection{Summary and Conclusions}

In conclusion, it has been observed that increases in the capillary temperature lead to a decrease in the average observed charge state of the protein cytochrome c. An increase in the voltage applied to the tube lens was seen to produce a similar effect. The trend toward lower charge states with increases in the tube lens offset voltage was seen to be stepwise, linear, and reproducible in the voltage range $0-60 \mathrm{~V}$. These changes are reasoned to be attributed to changes to the ions observed, not to the distribution of ions created. Both of the changes are thought to result from increased probability for chemical reactions at higher settings of the capillary temperature and tube lens offset. These reactions compromise to a greater extent the throughput of more reactive, higher charge ions, resulting in a bias toward lower charge states in the protein's full mass spectrum.

These findings might be seen as words of caution for those involved in protein conformational analysis by ESI/ITMS. It is clear from this research that the instrument parameters play a key role in what ions are observed, and changing of the settings can have a significant impact upon the charge state distribution of a protein. It should be noted from this 
work that a comprehensive evaluation of the instrument parameters on the CSD's should be thought of as a primary set of experiments when embarking on any conformational anaysis. Once optimized, the parameters should not be changed, as changing of these settings might cause a researcher to draw false conclusions about conformational changes of the proteins being studied. 


\section{Chapter 4: Influence of Sheath Liquid Composition on the Charge State Distributions of Heme Proteins}

\subsection{Introduction}

The mechanism of protein folding has long intrigued scientists involved in biological and biochemical research. The solution to the protein folding problem has eluded researchers for more than 30 years, though the lack of overall success has not discouraged work in the area, as the rewards for the problem's successful solution are great. A thorough understanding of the mechanisms of protein folding would lead to the ability to predict structure based solely upon primary sequence information. Therefore once a particular protein has been sequenced, its structure could be predicted. Information about three-dimensional structure then could be used to suggest the function and activity of the protein within the biological system.

As the Human Genome Project draws to a close and knowledge of the complete DNA sequence of man is at hand, the next logical step is to determine which proteins are coded for by the DNA, and to determine the activity of these proteins within the human body. Knowledge of the DNA sequence, and the patterns of mutation and other sequence anomalies and how they are manifested in the protein, will lead to an understanding of the genetic basis of disease. Contributions from protein folding research may make significant contributions to this effort.

The analytical sciences have made, and will continue to make contributions to the understanding of the protein folding process. Mass Spectrometry (MS), particularly that employing Electrospray Ionization (ESI), may play a central role. Since the advent of ESI-MS by Fenn and others in $1989^{5}$, this method has been widely applied to the analysis of protein structure. Fenn first observed the multiple charging of proteins that is characteristic of ESI. Later, Loo $^{9}$ observed that proteins are observed in the ESI spectrum as a collection of mass peaks 
with a varying number of associated protons, the ensemble of which is referred to as a Charge State Distribution (CSD). In the early 1990's, Chowdhury ${ }^{10}$ reported that the CSD exhibited by a protein could be shifted toward higher charge states by the addition of organic solvents. The shift was attributed to unfolding of the protein in the presence of the denaturing solvent that exposed basic amino acid residues in the hydrophobic core of the protein to the solvent for protonation, resulting in the observation of the protein at higher charge states in the ESI spectrum. This work suggested that solution-phase protein conformation could be monitored by ESI-MS. More recent work utilizing hydrogen-deuterium exchange has supported this assertion. ${ }^{40}$.

In the present work the influence of an organic sheath liquid on the charge state distributions of the heme-containing proteins myoglobin and cytochrome $\mathrm{c}$ is examined. The sheath liquid inlet in an electrospray ion source is sometimes used to provide to the source a solvent to mix with the analyte solution at the point of electrospray to improve the stability and sensitivity of the analysis. The analyte solution and the sheath liquid mix only at the capillary tip, where they are made into an aerosol by the action of the sheath gas. Thus it can be said that they are co-sprayed into a two-component aerosol at the capillary tip. It has been observed in this study that the nature of the sheath liquid utilized can have a dramatic effect upon the observed charge state distribution of a protein. The use of an organic liquid as sheath liquid causes significant shifts to higher charge states in the Charge State Distribution (CSD) of hemecontaining proteins. These shifts in the distribution are consistent with denaturation of the proteins in solution. The extent of the shift is related to the flow rate at which it is introduced to the ion source. 


\subsection{Materials and Methods}

Equine myoglobin and cytochrome c were purchased from Sigma (St. Louis, MO). HPLC-grade acetonitrile and methanol were obtained from Fisher Scientific (Fair Lawn, NJ). Infusions to the sheath liquid inlet were made by way of a syringe pump (KD Scientific,Boston, MA).

Connection between the syringe pump and a $250 \mu$ syringe (Hamilton, Reno, NV) was made by a length of PEEK tubing and plastic ferrules (Upchurch, Oak Harbor, WA).

Proteins were dissolved in deionized water from an in-house purification system.

Solutions were prepared at concentrations of $1.0 \times 10^{-5} \mathrm{M}$ and $2.0 \times 10^{-5} \mathrm{M}$ for cytochrome c and myoglobin, respectively. Samples in water were infused to the electrospray ion source of a ion trap mass spectrometer (Finnigan $\mathrm{LCQ}^{\circledR}$, ThermoQuest, San Jose, CA) at a rate of $3 \mu \mathrm{l}$ per minute for cytochrome c, $10 \mu \mathrm{l} / \mathrm{min}$. for myoglobin. Spectra were collected over the mass range $250-2000 \mathrm{AMU}$ for an acquisition time of 1 minute. All acquired scans were averaged to yield a spectrum for each analysis. Solvents were infused to the sheath liquid inlet at flow rates of 0 to $30 \mu 1 /$ minute. Nitrogen was used as sheath gas at a flow rate of 30 units/minute. The heated capillary was maintained at $175{ }^{\circ} \mathrm{C}$. A voltage of $4 \mathrm{kV}$ was applied to the stainless steel capillary tip, and the tube lens and octapole voltages were optimized to maximize ion transmission. 


\subsection{Results}

\section{Cytochrome c}

In the case of cytochrome $\mathrm{c}$, conformational changes induced by these conditions do not cause the dissociation of the prosthetic porphyrin group from the protein, as the heme unit is covalently bound to the porphyrin through two covalent linkages. The presence of a denaturing solvent as sheath liquid does, however, cause significant changes to the charge state envelope of the protein, suggesting a conformational change during the electrospray/desolvation process. Figures 4.3.1 and 4.3.2 show the mass spectra of cytochrome $\mathrm{c}$ in deionized water with a sheath liquid of acetonitrile at flow rates of 0 and $20 \mu \mathrm{l} /$ minute, respectively.

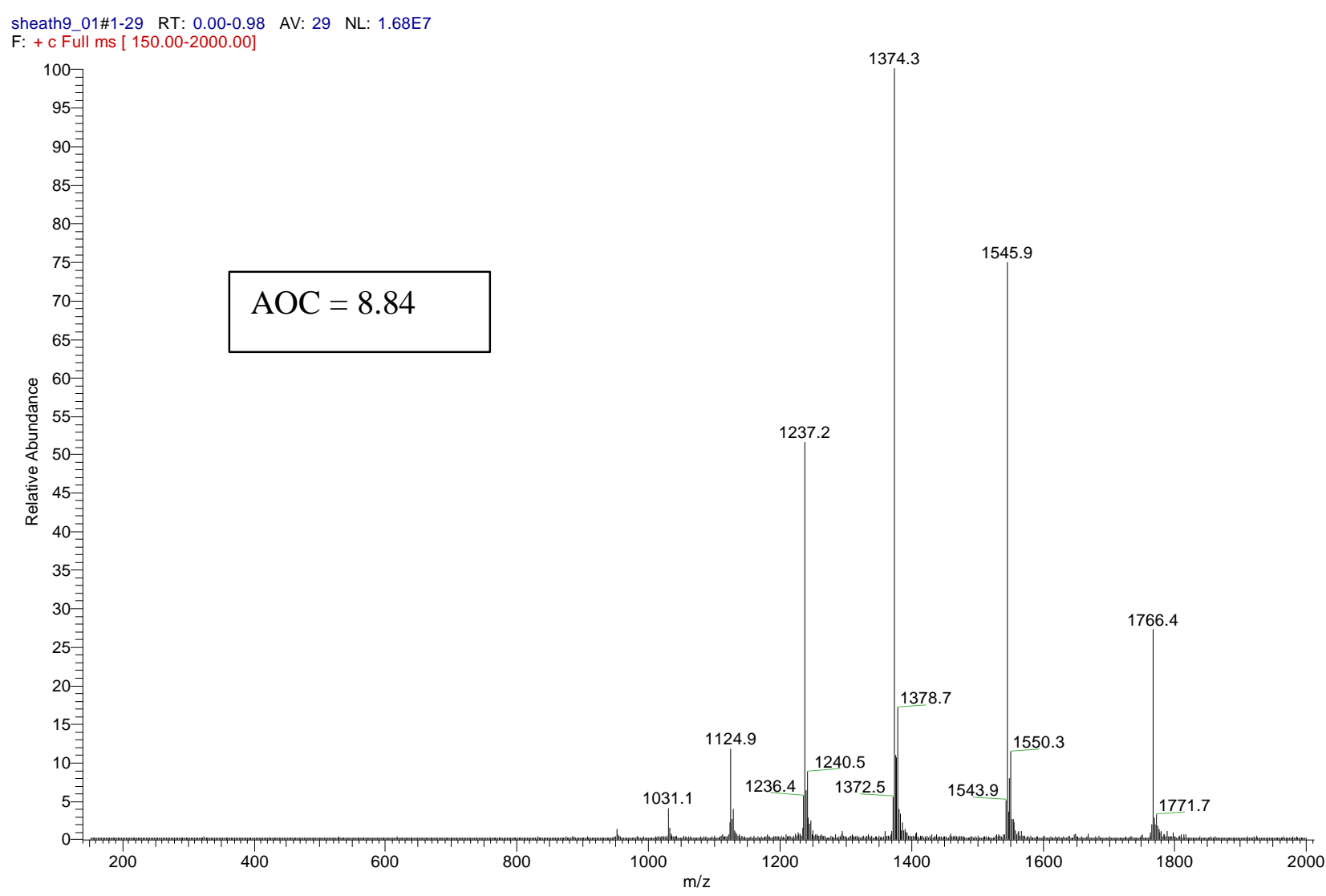

Figure 4.3.1: Mass Spectrum of Cytochrome c, Acetonitrile Sheath Liquid Flow Rate $=0 \mu \mathrm{l} / \mathrm{min}$. 


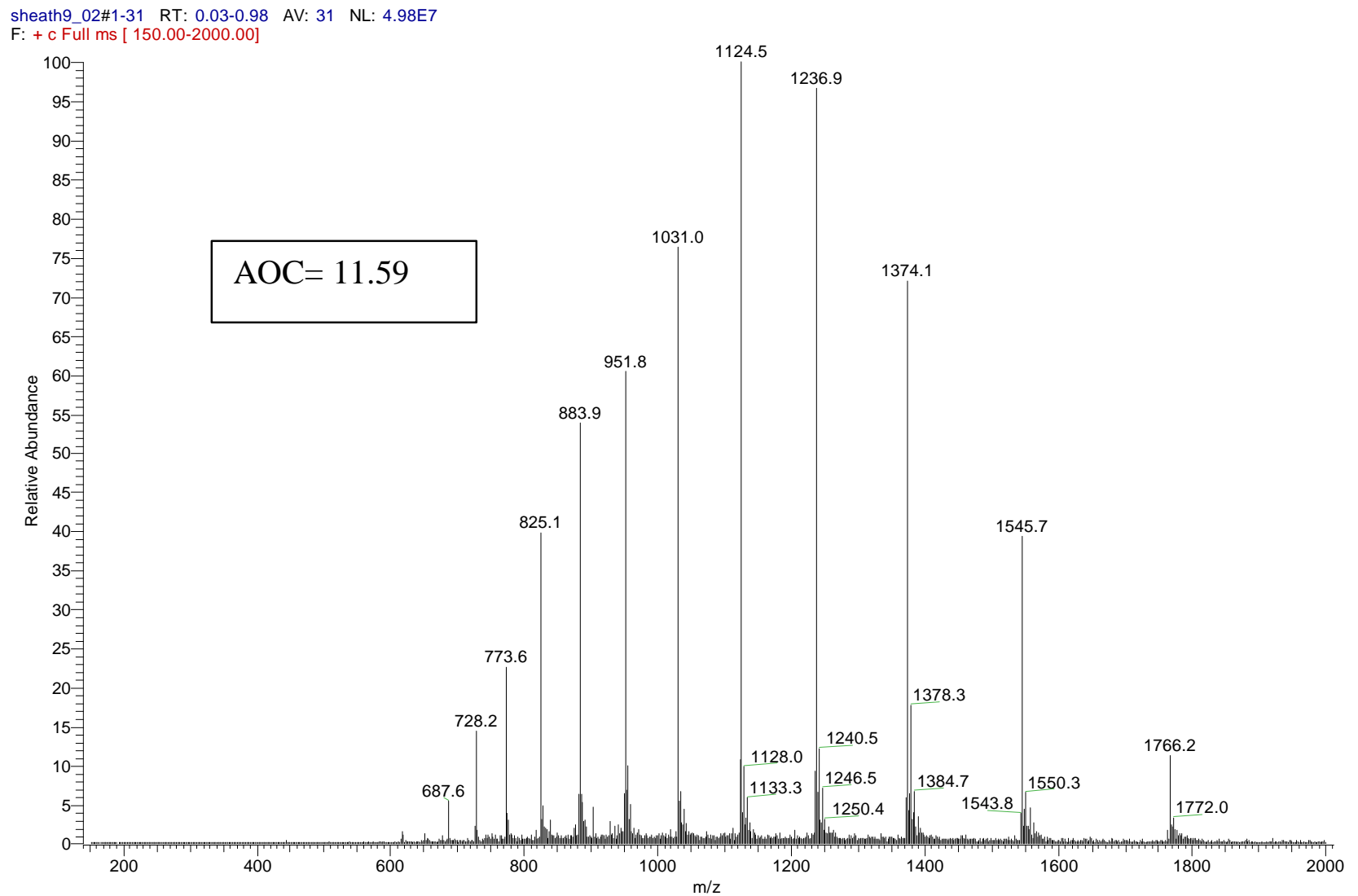

Figure 4.3.2: Mass Spectrum of Cytochrome c, Acetonitrile Sheath Liquid Flow Rate $=20 \mu \mathrm{l} / \mathrm{min}$. 


\section{Myoglobin}

In the case of myoglobin, the introduction of an organic sheath liquid results in very

dramatic shifts in the spectra when compared to that acquired in the absence of sheath liquid.

Because the prosthetic heme group is not covalently attached to the protein as in cytochrome $\mathrm{c}$

and is merely ligated to the protein backbone, interaction with the organic sheath during the process of electrospray causes $50 \%$ dissociation of the heme group from the protein. This results

in the formation of apomyoglobin and the liberation of the heme group, observed at m/z 616 .

Figures 4.3.3-4.3.7 show the mass spectra of myoglobin in deionized water with acetonitrile

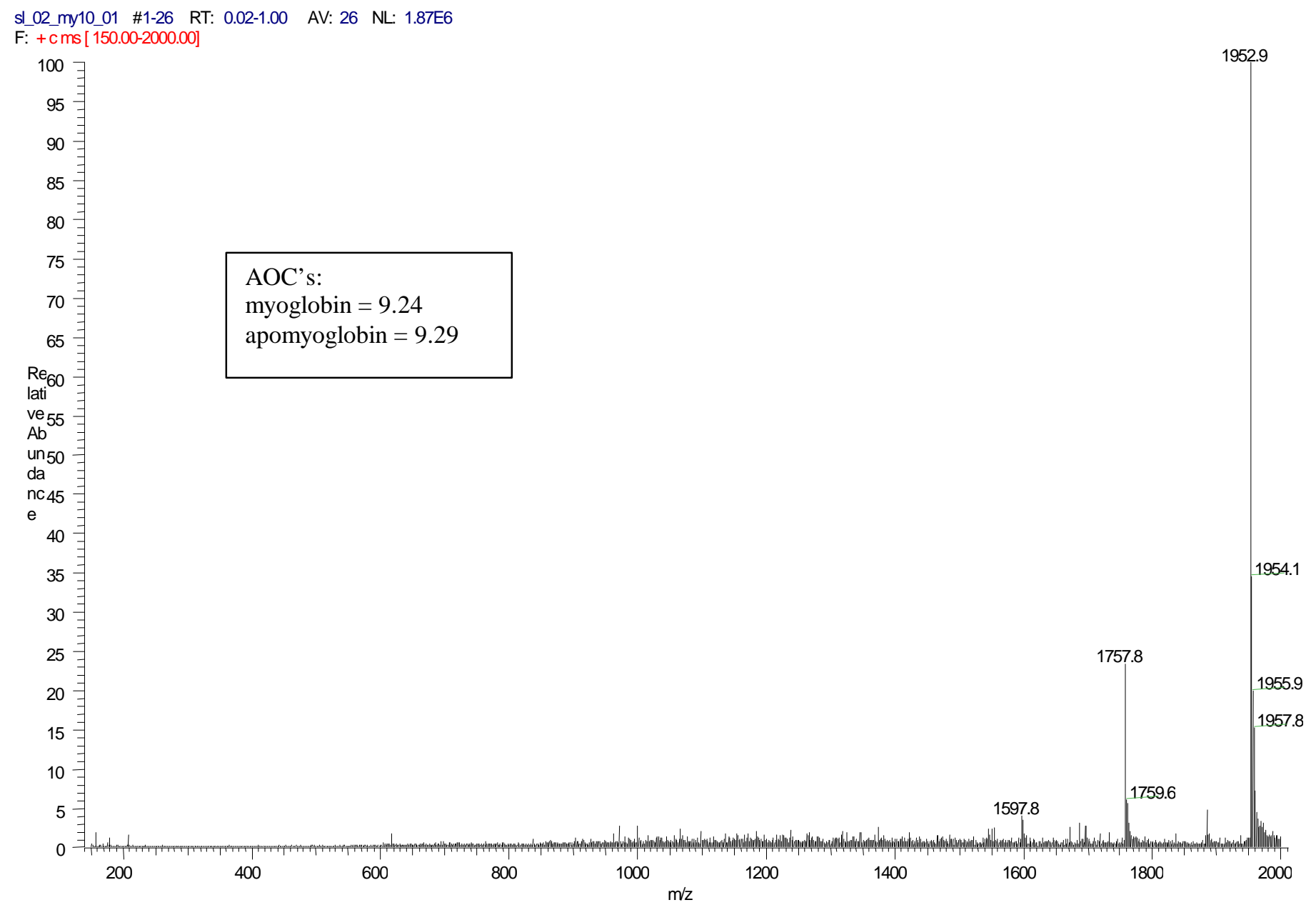

Figure 4.3.3: Mass Spectrum of Myoglobin, Acetonitrile Sheath Liquid Flow Rate $=0 \mu \mathrm{l} / \mathrm{min}$. 
sheath liquid flow rates of 0,5 , and 10, 20, and30 $\mu 1 /$ minute. In Figure 4.3.3, only myoglobin molecules with the heme group intact (holomyoglobin) are observed. Upon the introduction of sheath liquid flow (Figure 4.3.4), the heme group begins to dissociate from the holoprotein and is observed in the spectrum as a singly charged species at $\mathrm{m} / \mathrm{z} 616$. Because the dissociation is incomplete, a doublet of peaks is evident for each charge state, one for the holomyoglobin at higher mass, and one corresponding to apomyoglobin at lower mass.

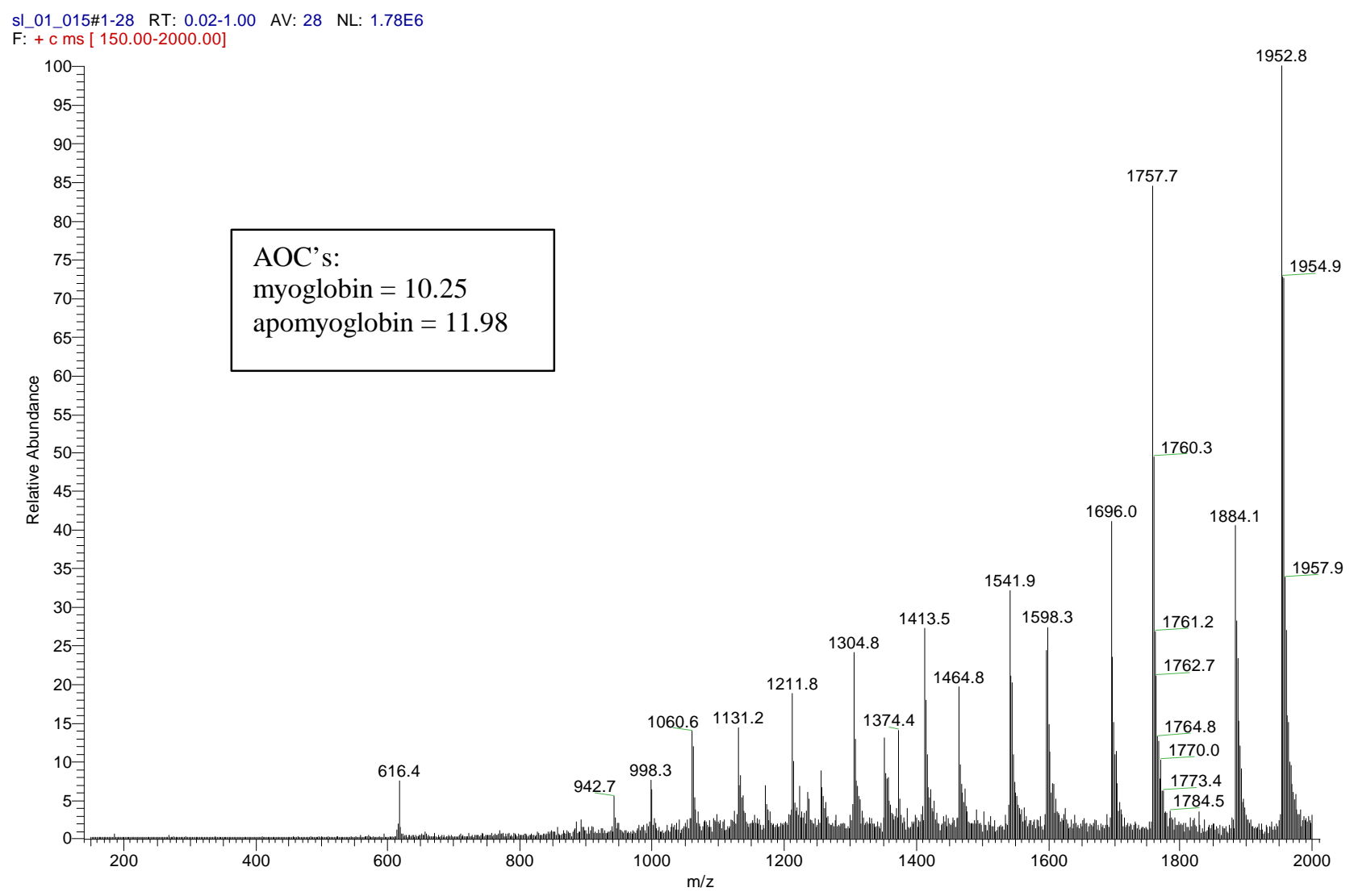

Figure 4.3.4: Mass Spectrum of Myoglobin, Acetonitrile Sheath Liquid Flow Rate $=5 \mu \mathrm{l} / \mathrm{min}$. 


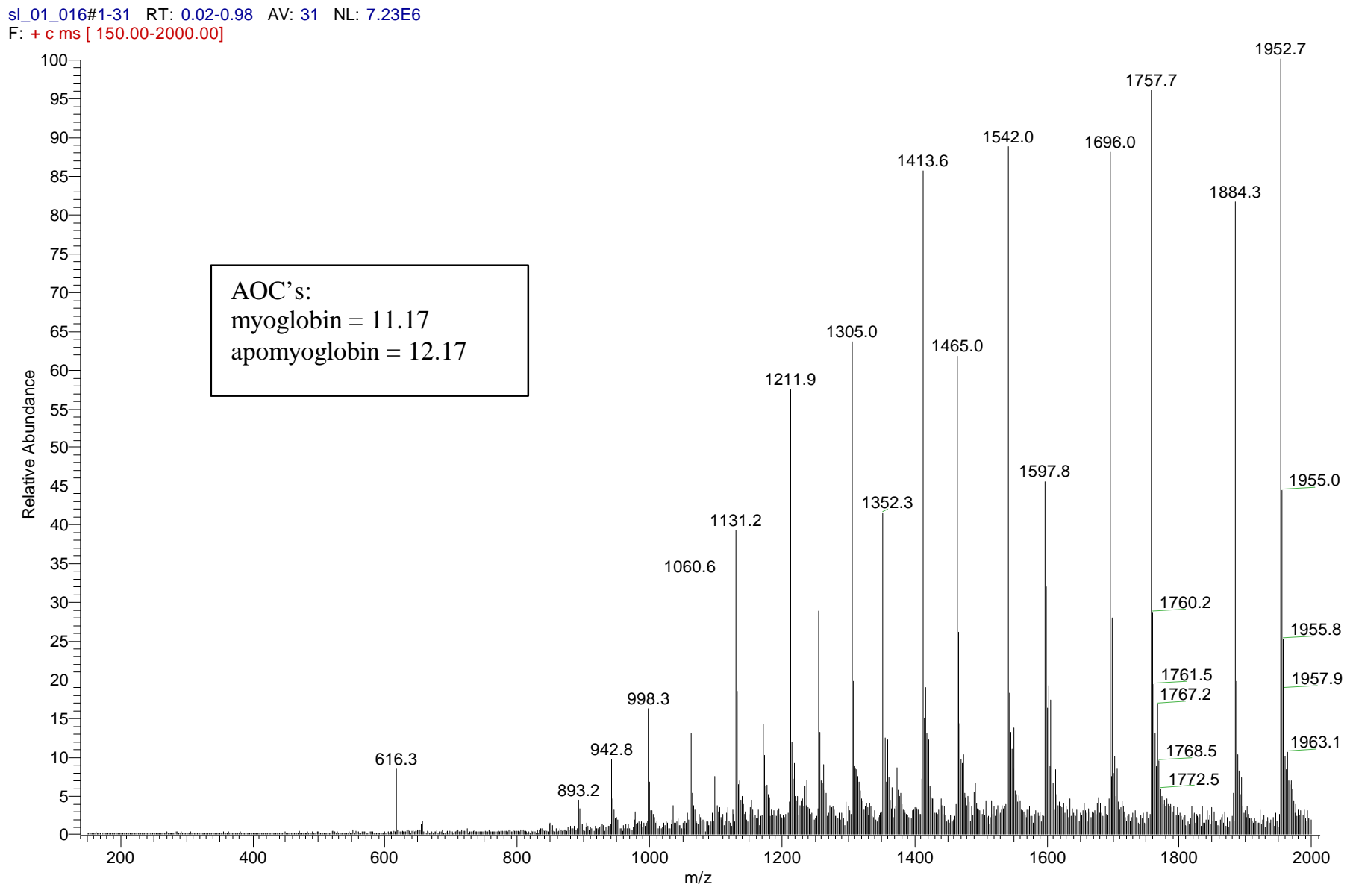

Figure 4.3.5: Mass Spectrum of Myoglobin, Acetonitrile Sheath Liquid Flow Rate $=10 \mu \mathrm{l} / \mathrm{min}$. 
sl_01_017\#1-31 RT: 0.03-0.98 AV: 31 NL: 9.92E6

$\mathrm{F}:+\mathrm{C}$ ms [ 150.00-2000.00]

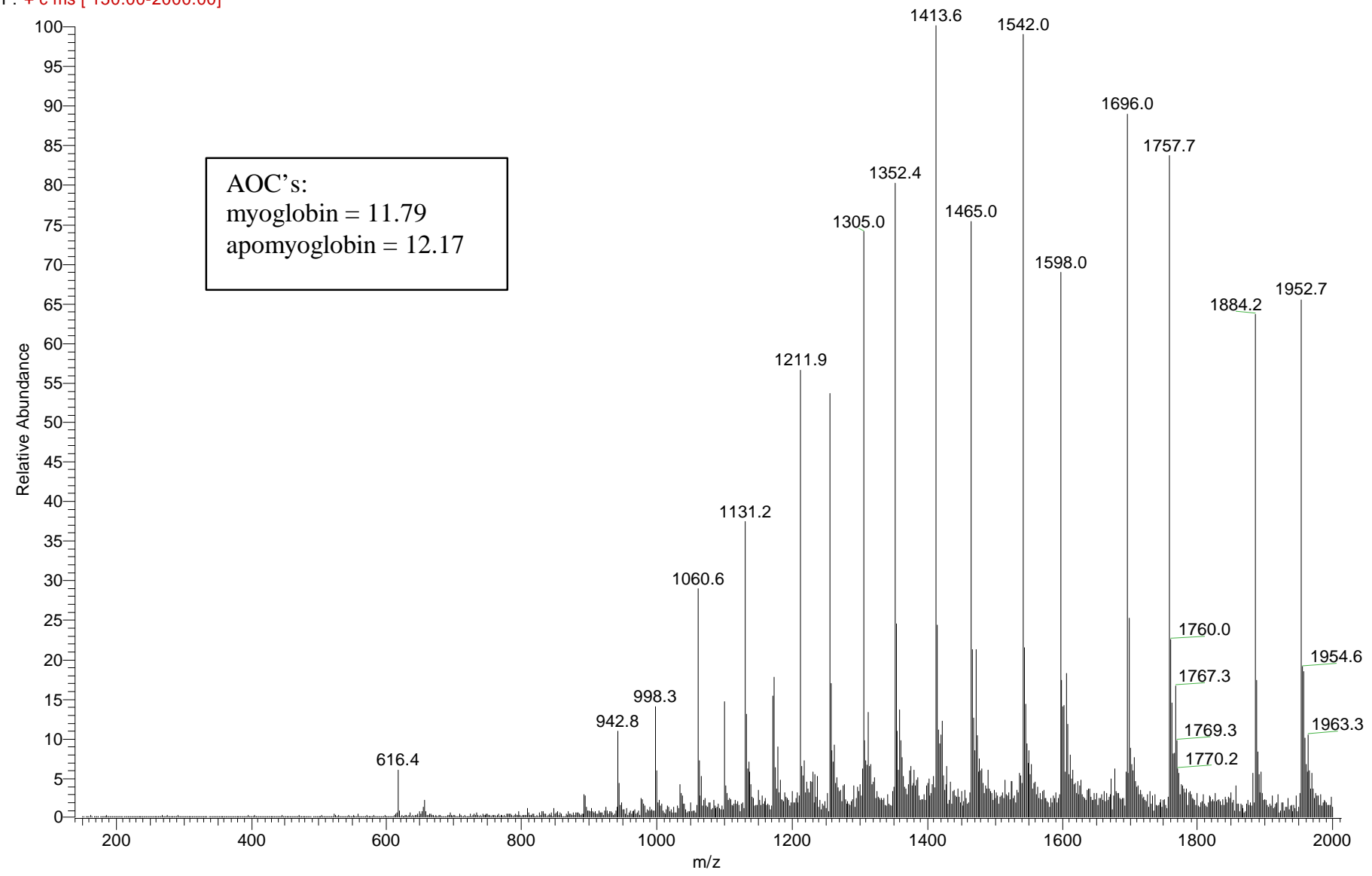

Figure 4.3.6: Mass Spectrum of Myoglobin, Acetonitrile Sheath Liquid Flow Rate $=20 \mu \mathrm{l} / \mathrm{min}$. 


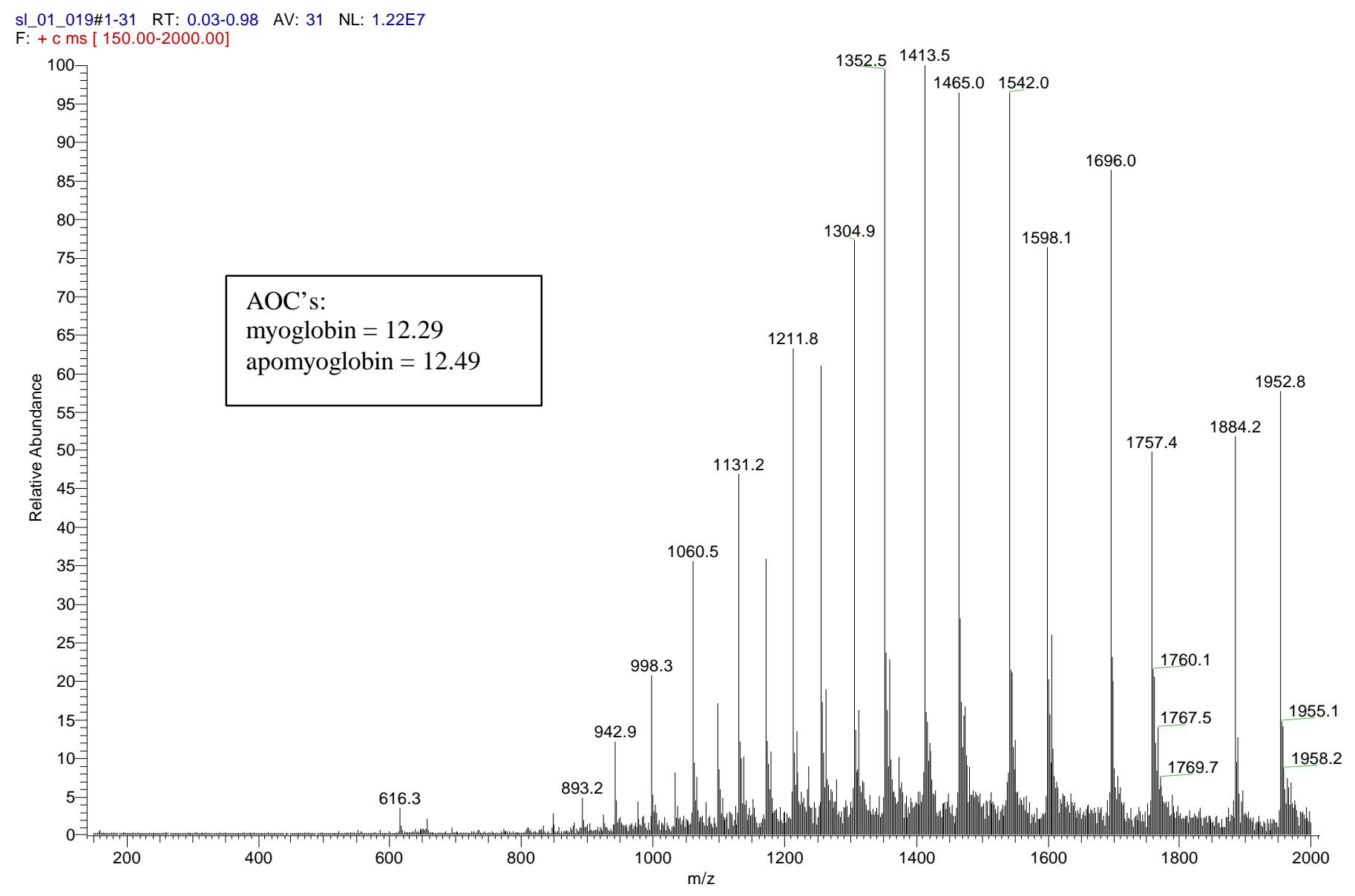

Figure 4.3.7: Mass Spectrum of Myoglobin, Acetonitrile Sheath Liquid Flow Rate $=30 \mu \mathrm{l} / \mathrm{min}$. 
In the case of cytochrome $\mathrm{c}$, the denaturing influence of the sheath liquid causes significant shifts in the CSD. In the absence of sheath flow (Figure 4.3.1), the Average Observed Charge (AOC) is calculated to be 8.84 , with the $7+$ through $9+$ charge states populated. At a sheath flow of $20 \mu \mathrm{l} / \mathrm{min} /$, the AOC is calculated at 11.59 and the $7+$ through $18+$ charge states are in evidence. Therefore the sheath liquid flow induced a change in the AOC of +2.75 , and resulted in the population of six charge states that were not evident in the absence of organic sheath liquid. However the induced unfolding of the protein does not cause the dissociation of the heme group from the structure, as it as covalently bonded to the polypeptide chain.

Similar effects are observed for myoglobin. As was previously indicated, the influence of the sheath liquid causes partial dissociation of the heme group from the holoprotein, and subsequent formation of apomyoglobin. Thus the conformational changes of both myoglobin and apomyoglobin induced by the sheath flow may be observed. Figure 4.3 .8 shows the dependence of the AOC on the sheath liquid flow rate for myoglobin and apomyoglobin. Figure 4.3.9 shows the populations of myoglobin and apomyoglobin at various flow rates. From the plots it can be seen that the myoglobin is rapidly converted to apomyoglobin by the loss of the prosthetic heme group, even at low sheath liquid flow rates. It can also be noted in Figure 4.3.8 that while apomyoglobin achieves a near maximum $\mathrm{AOC}(11.98)$ at a flow rate of only $5 \mu \mathrm{l} / \mathrm{minute}$, the AOC of myoglobin continues to increase steadily throughout the flow regime. This observation suggests that upon dissociation of the heme group from the intact holoprotein, the resulting apomyoglobin rapidly unfolds. Once the rigid, planar porphyrin ring is lost from the holoprotein, much of the overall structural integrity of the molecule is compromised, and the remaining apoprotein readily unfolds. The unfolding of intact myoglobin would appear to be a slower process. Those molecules wherein the heme group remains in place do not readily unfold, and 
their progression to higher charge states by unfolding is a more gradual process. It seems then that the prosthetic group in myoglobin plays an important role in the preservation of tertiary structure. The presence of the heme group imparts conformational stability to the molecule. When it is absent from the structure, much of the structural integrity of myoglobin is compromised. 
Average Observed Charge of Myoglobin and Apomyoglobin

vs.Acetonitrile Sheath Liquid Flow Rate

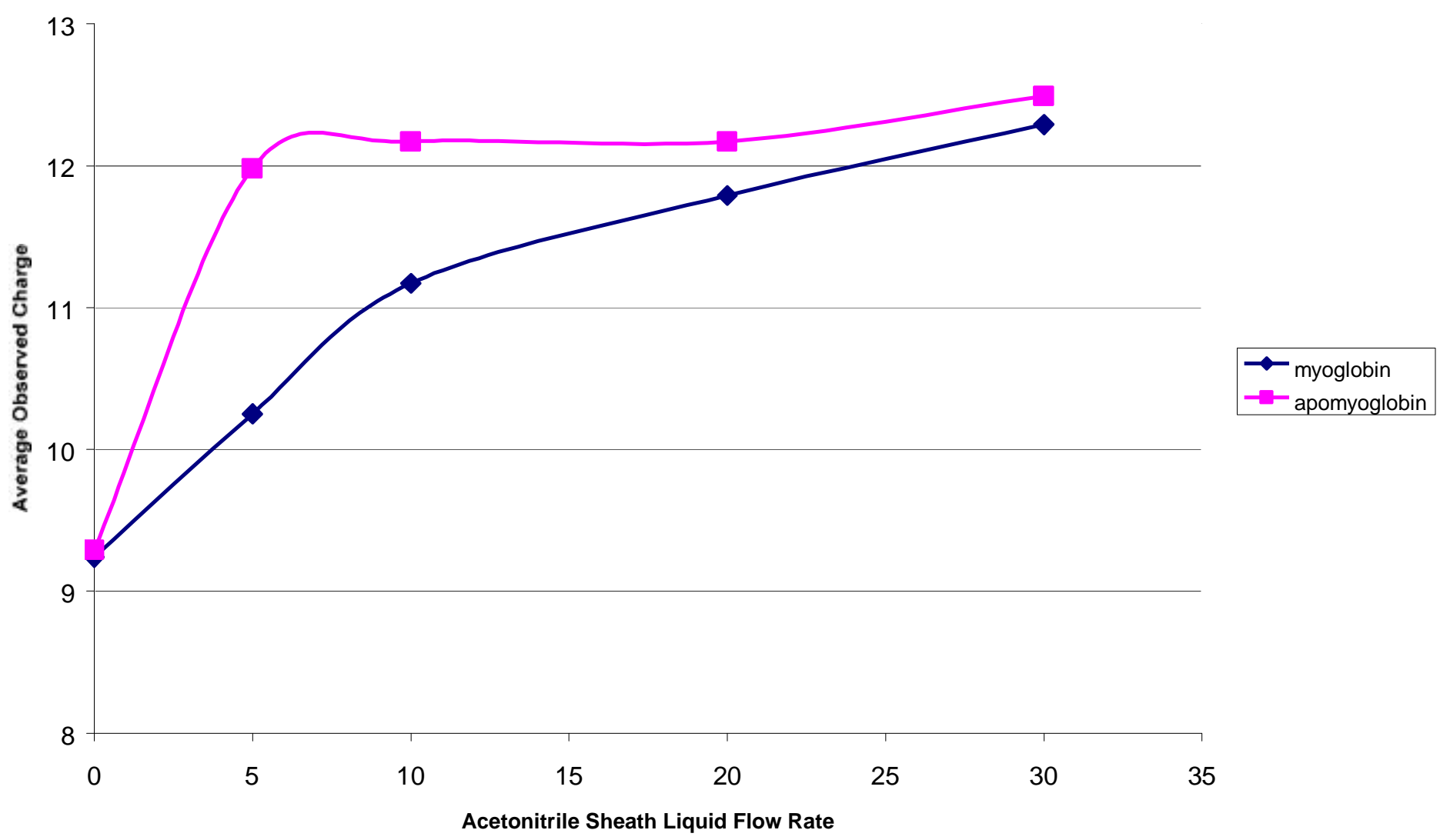

Figure 4.3.8: Average Observed Charge of Myoglobin and Apomyoglobin vs. Acetonitrile Sheath Liquid Flow Rate 


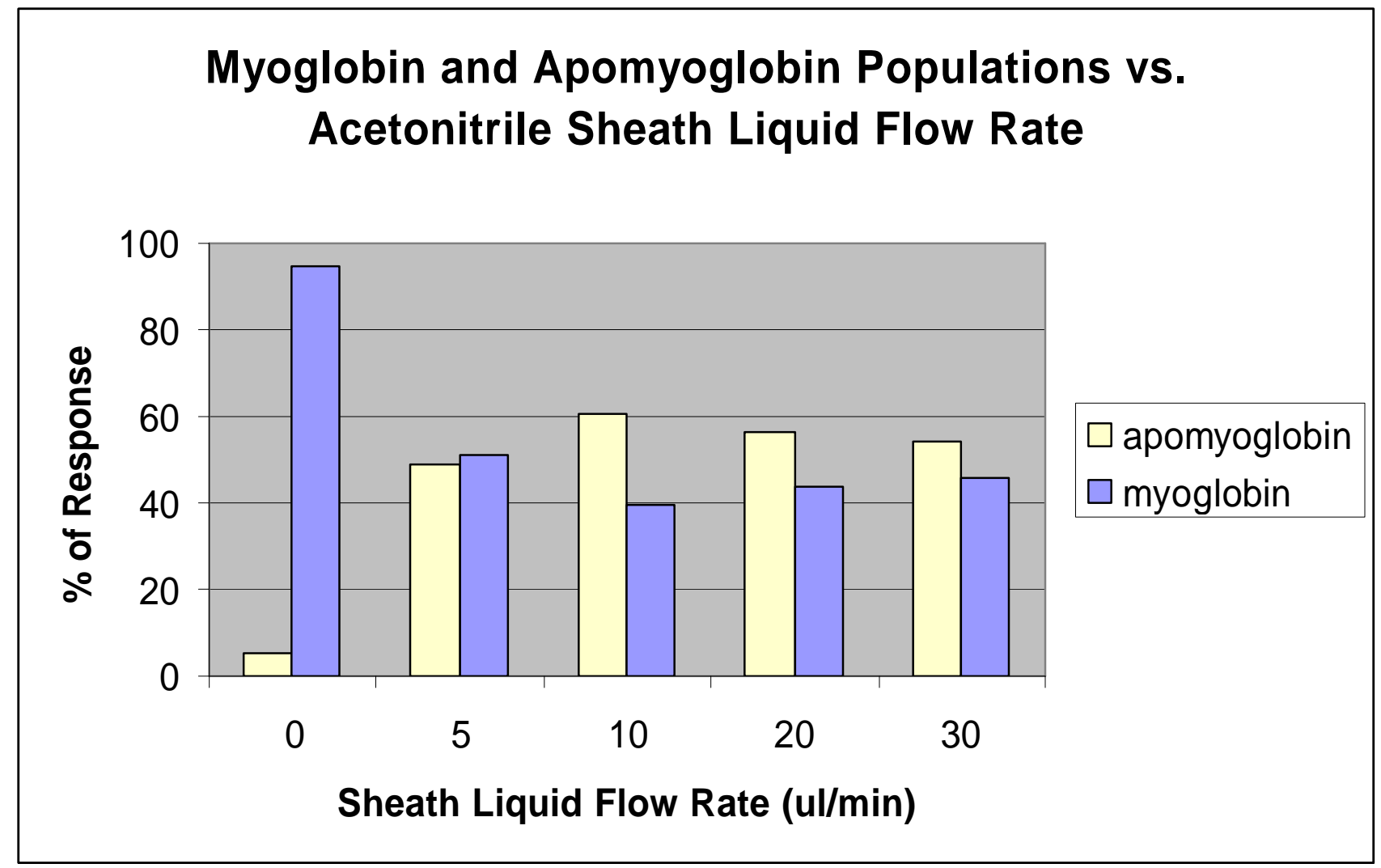

Figure 4.3.9: Percentage of Response Due to Myoglobin and Apomyoglobin at Various Acetonitrile Sheath Liquid Flow Rates. 


\subsection{Discussion}

The results clearly show that the introduction of an organic sheath liquid significantly alters the observed Charge State Distribution (CSD) of both proteins. It is well known that the addition of organic liquids to aqueous solutions of proteins results in denaturation. This is observed in the mass spectrum as a shift to higher charge states from lower charge states observed in the absence of denaturing conditions $\mathrm{s}^{20}$. This effect is reasoned to result from unfolding of the protein by the disruption of hydrogen bonds that exposes basic residues along the protein to the solvent environment for protonation, resulting in the observation of the protein in the mass spectrum at lower $\mathrm{m} / \mathrm{z}$ values, and an overall shift in the CSD to higher charge states. The presence of an organic sheath liquid that is co-electrosprayed with the aqueous protein solution would seem to have a similar effect.

The organic sheath liquid and the aqueous protein solution are permitted to mix only at the end of the capillary tip, where they are co-sprayed into an aerosol. Therefore the changes to the CSD's observed must be attributed to changes to the protein structure at the electrospray capillary tip, and during the formation of the aerosol and subsequent desolvation, but before it reaches the gas phase. The interaction of the protein molecules with the organic liquid within capillary tube, and in the aerosol beyond the electrospray needle seems to have the same effect as the addition of bulk organics to protein solutions. This means that the time of interaction within the capillary in addition to the droplet lifetime must be sufficiently long as to allow changes to protein conformations within the droplets before the desolvation process is complete and the ions enter the gas phase.

Droplet lifetime is estimated to be on the order of a few hundred microseconds to a few milliseconds, depending upon the type of ion source used ${ }^{46}$. Additional time for interaction may 
be afforded during the formation of the Taylor cone. The Taylor cone is formed at the end of the electrospray needle, as positively charged ions move away from the needle to reduce repulsive forces. Because the Taylor cone extends past the needle, droplets of organic liquid could combine with the aqueous solution in the cone, beginning the process of denaturation of protein molecules before the evolution of droplets from the Taylor cone. However the organic sheath liquid would serve to destabilize the cone, as the merging of the organic solvent with the aqueous solution would reduce the surface tension of the liquid. Protein unfolding reactions are known to be processes of greatly varying rates, with durations of nanoseconds to hours ${ }^{47}$. It is possible then that the changes seen are due to the interaction of the organic sheath liquid within the droplets, which induces conformational changes to the protein molecules, resulting in changes to the observed Charge State Distribution

As an application note, the technique of mixing solutions at the capillary tip by way of the sheath liquid inlet might be useful for observing other phenomena by ESI-MS. A group of metal ions, or pharmaceutical compounds might be dissolved in the sheath liquid and electrosprayed along with a protein such as an enzyme of receptor. As the interaction of rapidacting binding agents could be observed, a series of potential protein "activators" might be screened for binding affinity simultaneously. This would allow for the rapid screening of potential protein activators, and the ability to observe the binding of numerous agents simultaneously. 


\subsection{Conclusions}

It can be seen from this work that the use of an organic sheath liquid can significantly alter the Charge State Distribution (CSD) of a protein. The extent of the change in the Average Observed Charge (AOC) is related to the flow rate of the sheath liquid utilized. The changes observed are attributed to conformational changes of the proteins that occur at the electrospray capillary tip, and during the process of droplet evolution and subsequent ion desolvation. Therefore we conclude that conformational changes are rapid processes, and that significant conformational changes can occur during droplet evolution and ion desolvation if exposure to denaturing agents occurs during the process of electrospray.

Additionally, it is observed that the presence of the heme group in myoglobin imparts structural integrity to the protein and an ability to resist conformational changes. While the globin portion of the molecule serves to protect the heme group from the solvent environment where it might be damaged by oxidative/reductive forces, the heme group serves to anchor the molecule together, maintaining the tertiary structure of myoglobin. 


\section{Chapter 5: Energetics of Heme Dissociation from Heme-Containing Proteins: Evidence of the Correlation Between Charge States and Conformational States in the ESI/MS Analysis of Proteins}

\subsection{Introduction}

Since the advent of electrospray ionization and its application to the analysis of proteins, a lively debate has persisted as to whether the charge states of a protein as observed by mass spectrometry are reflective of the protein's conformation, that is the three-dimensional shape of the protein. Also at issue has been the question as to whether solution-phase protein conformations are preserved after transfer to the gas-phase by the process of electrospray. While most early studies of protein conformations by ESI-MS ${ }^{910}$ concluded that the charge state envelope exhibited by a protein is indicative of conformation, other workers strongly rebuffed this assertion ${ }^{48}$. Recent work utilizing hydrogen-deuterium exchange suggests that the charge state envelope of a protein does reflect the protein's conformation ${ }^{49}$. Additionally, other recent research in the area has concluded that significant protein denaturation does not take place during the process of electrospray ionization ${ }^{50}$.

In the case of the heme-containing proteins, if the higher charge states of proteins as observed by mass spectrometry do represent more unfolded forms, this unfolding might expose the heme group to the surrounding environment. Such exposure of the prosthetic heme might then labilize the heme:protein association making it easier to liberate the porphyrin from the structure, suggesting an inverse relationship between increasing charge state and the energy required for heme dissociation. This relationship was observed by Hunter and others ${ }^{51}$ for the heme-containing proteins myoglobin and cytochrome $b_{5}$ in performing source CID on triplequadrupole instruments. In these studies, the authors compared the intensity of the undissociated 
holomyoglobin $(\mathrm{n}+)$ to that of the apomyoglobin $([\mathrm{n}-1]+)$, the most prominent mass observed upon dissociation of a given charge state. By increasing the collision energy in order to achieve $50 \%$ dissociation, they were able to determine activation energies for the dissociative processes for the various charge states of the two studied proteins. Their results suggested the expected inverse relationship between increasing charge state and the energy required to dissociate the heme group from the structures of the heme proteins myoglobin and cytochrome b5. This approach, however, neglects the formation of the $\mathrm{n}+$ charge state of apomyoglobin that also results from CID of heme proteins. Also, the two proteins were electrosprayed from different solvent environments, so direct comparisons between the proteins were not possible. And the analysis did not include proteins in which the porphyrin is covalently linked to the protein.

In this work we employ a different approach to the problem. Using an ion trap mass spectrometer, we first isolate individual charge-states within the trap, then perform CID within the ion trap and monitor the intensity of the heme ion response. This is done at variable collision energies to obtain plots of heme ion intensity vs. applied collision energy for each charge state. These experiments are performed for myoglobin, in which the heme group is not covalently attached, and are extended to cytochrome $\mathrm{c}$, wherein the heme is covalently bonded to the protein. As the proteins are electrosprayed from identical solution environments, direct comparisons between them are possible.

Heme proteins are an important class of proteins that perform many critical functions in biological systems, Cytochrome c, is a $12 \mathrm{kD}$ protein consisting of a single polypeptide chain associated with an iron-containing porphyrin core referred to as the heme group. It is found primarily in bacterial cells, particularly within mitochondrial membrane walls where it serves as an electron carrier that facilitates oxidation and reduction reactions vital to cellular respiration. 
The heme group present in Cytochrome $\mathrm{c}$, of the ferroprotoporphyrin IX type, is associated to the protein by coordination of the Met- 80 and His-18 residues of the protein to the metal center and through two thioether bonds between the termini of the porphyrin ring and Cys-14 and Cys-17 residues of the protein. Myoglobin is found in mammalian muscle tissues, where it serves as an oxygen storage sink. It consists of a globular protein portion surrounding a heme core, also of the ferroprotoporphyrin IX type. However in myoglobin the prosthetic heme group is not covalently associated with the protein portion of the molecule, but is held by coordination of the His-93 residue of the protein to the metal center, by hydrogen bonding and by van der Waals interaction. ${ }^{52}$ Though the heme group in the two proteins is of the same type, the association of the heme group and the protein differs significantly. Owing to the fact that covalent interactions are stronger than the types of weak interactions that tether the heme group to the protein in myoglobin, the heme-protein interaction should in theory be stronger than that in myoglobin. This work has endeavored to observe the difference in energy required for heme dissociation from the two proteins.

Additionally, within each protein if the charge states observed by ESI-MS are reflective of the conformational state of the protein in solution, then higher charge states represent more unfolded forms of the protein, as the unfolding exposes basic amino acid residues to the solvent environment where they may be protonated. The exposure of such sites and subsequent protonation then results in the appearance of the protein at a higher charge state in the mass spectrum. In the case of the heme proteins studied, such unfolding might expose the heme group to CID, making collisions more effective at dislodging the heme group from the structure. If this is true, then the heme group should be more readily dissociated from higher charge states than 
from lower ones, that is an inverse relationship would exist between increasing charge state and the energy required to liberate the heme group from the protein.

This work examines the energy required for heme dissociation from each charge state of cytochrome $\mathrm{c}$ and myoglobin to determine if an inverse relationship exists between increasing charge state and the energy required for heme dissociation in these two protein systems. The like charge states of the two proteins were compared in an effort to determine if the covalent linkages present in cytochrome $\mathrm{c}$ between the protein and the prosthetic heme group require more energy to disrupt, and thus dislodge the heme group, than is required to liberate the same group from myoglobin, in which the protein is not covalently associated with the prosthetic heme group.

\subsection{Materials and Methods}

Horse skeletal myoglobin and bovine cytochrome c were obtained from Sigma, and were used without purification. Solutions of the proteins were prepared in deionized water at concentrations of $125 \mu \mathrm{g} / \mathrm{ml}$ (myoglobin) and $100 \mu \mathrm{g} / \mathrm{ml}$ (cytochrome c). Spectra were acquired on a Finnigan

LCQ Mass Spectrometer fitted with a standard electrospray ion source. Samples were infused to the ion source by syringe pump at a flow rate of $3 \mu 1 / \mathrm{min}$.

Instrument conditions were optimized in order to observe the maximum number of charge states of holoprotein while exhibiting sufficient sensitivity to perform the desired analyses. Once established, the same conditions were used for the analysis of all charge states of both proteins. Thus all of the data shown were obtained under identical conditions so that direct comparisons can be made.

In order to evaluate heme dissociation from the holoproteins, a full spectrum of the protein was first acquired. For each charge state, a 3 AMU window was constructed around the 
most abundant mass to isolate the selected charge state within the ion trap. MS/MS analyses in the Selected Reaction Monitoring (SRM) mode were then performed at relative collision energies of $10 \%-40 \%$, in $2 \%$ increments. $\mathrm{SRM}$ at $\mathrm{m} / \mathrm{z}=616$ was used for myoglobin, and SRM at $\mathrm{m} / \mathrm{z}=617$ for cytochrome $\mathrm{c}$. These $\mathrm{m} / \mathrm{z}$ values correspond to the masses of the heme group liberated from the respective proteins. The assignment of $\mathrm{m} / \mathrm{z}=616$ for the heme unit dissociated from myoglobin, and $\mathrm{m} / \mathrm{z}=617$ for the heme unit liberated by CID from cytochrome $\mathrm{c}$ are well documented in the literature ${ }^{14,12}$. SRM data were acquired for 3 minutes at each $\%$ relative energy setting for each charge-state. Spectra were averaged to give a value for the heme ion intensity for each acquisition. Within each charge state, intensities were normalized to the largest observed intensity in order to scale responses to values between 0 and 1 . The normalized intensities were then plotted vs. \% relative collision energy to obtain the heme dissociation plots for each charge state of each protein. 


\subsection{Results and Discussion}

Figure 5.3.1 shows the heme dissociation plots for myoglobin, and Figure 5.3.2 shows the analogous plot for cytochrome c. A stepwise increase in energy is required for the

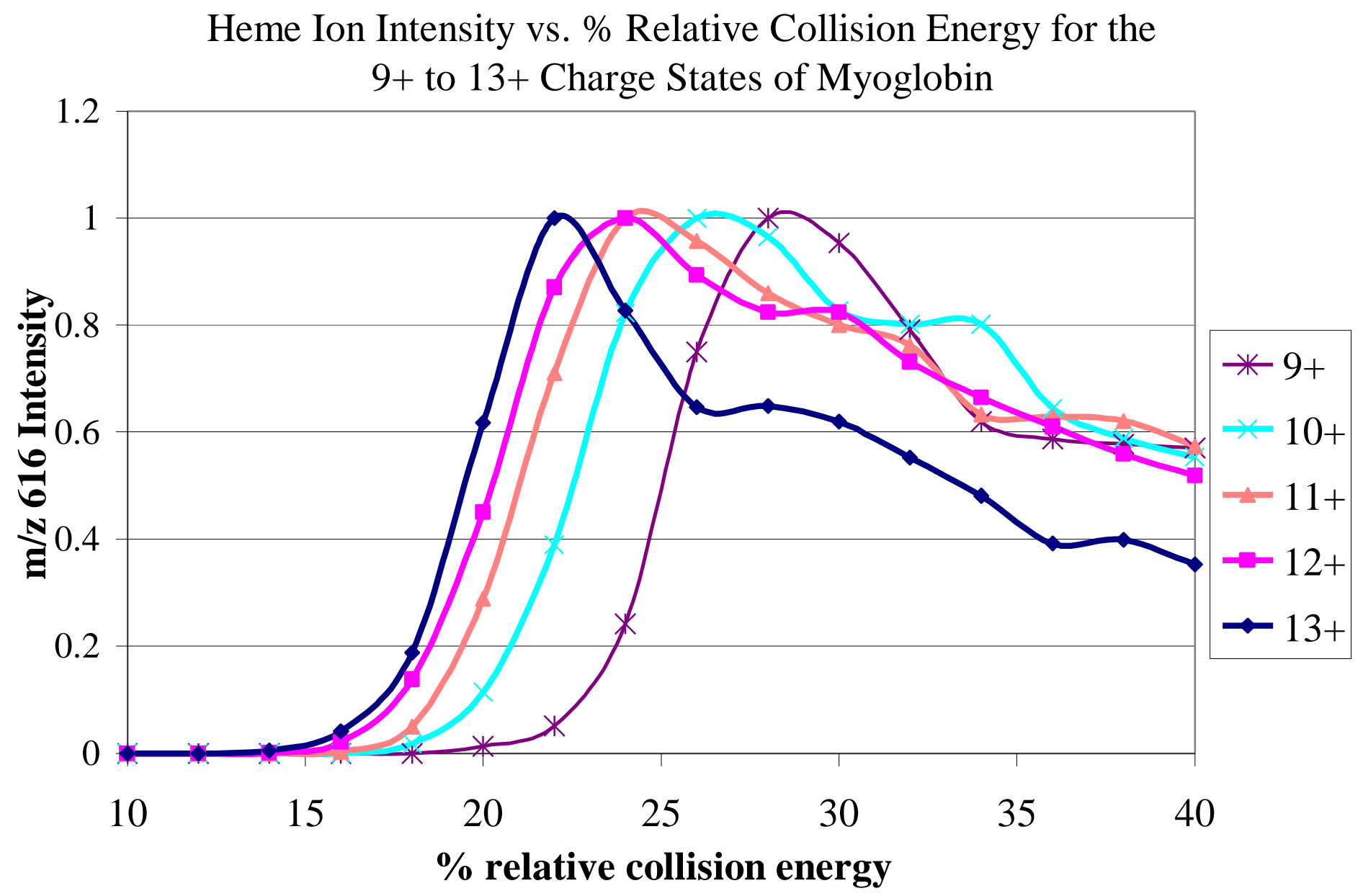

Figure 5.3.1: Heme Ion Intensity versus \% Relative Collision Energy for the 9+ to 13+ Charge States of Myoglobin

threshold of heme dissociation with decreasing charge state for both proteins. In myoglobin, it is observed that the maximum heme ion intensity is observed at higher energies with each decrease in charge state. 
Heme Ion Intensity vs. \% Relative Collision Energy for the $7+$ to $11+$ Charge States of Cytochrome c

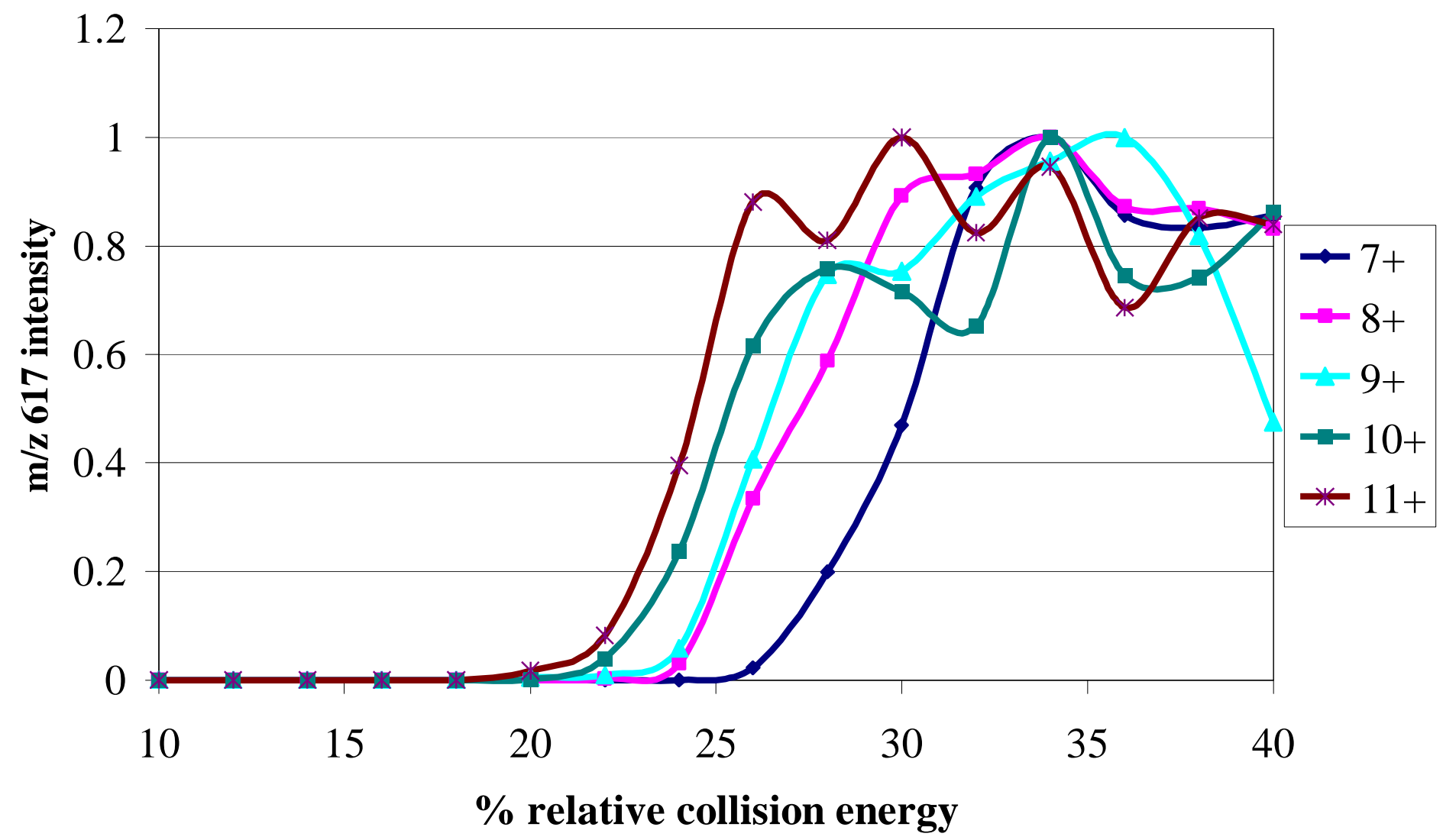

Figure 5.3.2: Heme Ion Intensity versus \% Relative Collision Energy for the 7+ to 11+ Charge States of Cytochrome c.

The increased energy demand required to observe the threshold of heme dissociation with decreasing charge state is observed for both proteins. These results are consistent with the idea that increased charge states of a protein represent more unfolded forms. The unfolding of the protein exposes the heme group to CID in the gas phase within the ion trap. As a consequence of the unfolding, and the resultant exposure of the heme, collisions with helium within the trap are more effective at dislodging the heme group from the structure. These results also support the assertion that solution-phase behavior is preserved after transfer to the gas phase by electrospray. It is known from $\mathrm{CD}^{53} 54$ (Circular Dichroism) measurements that proteins studied under 
denaturing conditions in solution, the same conditions that generate higher charge states in the mass spectrum, represent the more unfolded forms of a protein. These results are also consistent with solution hydrogen-deuterium (HD) exchange experiments ${ }^{32,55}$. In these experiments it is observed that more unfolded forms of a protein undergo a greater number of exchange reactions than do tightly folded forms. This is thought to be due to the exposure of basic sites to the solvent environment by the unfolding process. Basic residues along the protein backbone that are hindered in tightly folded states are rendered accessible to solvent for exchange by the unfolding process. Thus the more unfolded states the protein can accept a higher number of protons, and higher charge states in the mass spectrum result. Taken together with CD measurements and H-D exchange data, our results support the assertion that the protein conformations generated by the solution environment are preserved after transfer to the gas phase by the process of electrospray.

In order to make a comparison between like charge states of the two proteins, the collision energies were corrected for the mass difference between the two proteins by dividing the $\%$ relative collision energy by the mass of the protein. The normalized intensities of the heme ion response for the $9+, 10+$, and $11+$ charge states were then plotted versus the relative collision energy per unit mass. In comparing the like charge states, bias in the results due to the destabilizing effects of charge is avoided. Figure 5.3.3 shows a mass-corrected comparison of the dissociation plots for $9+$ to $11+$ charge states of the two proteins. 
Heme Ion Intensity vs. \% Relative Collision Energy/Mass for the 9+ to 11+ Charge States of Myoglobin and Cytochrome c

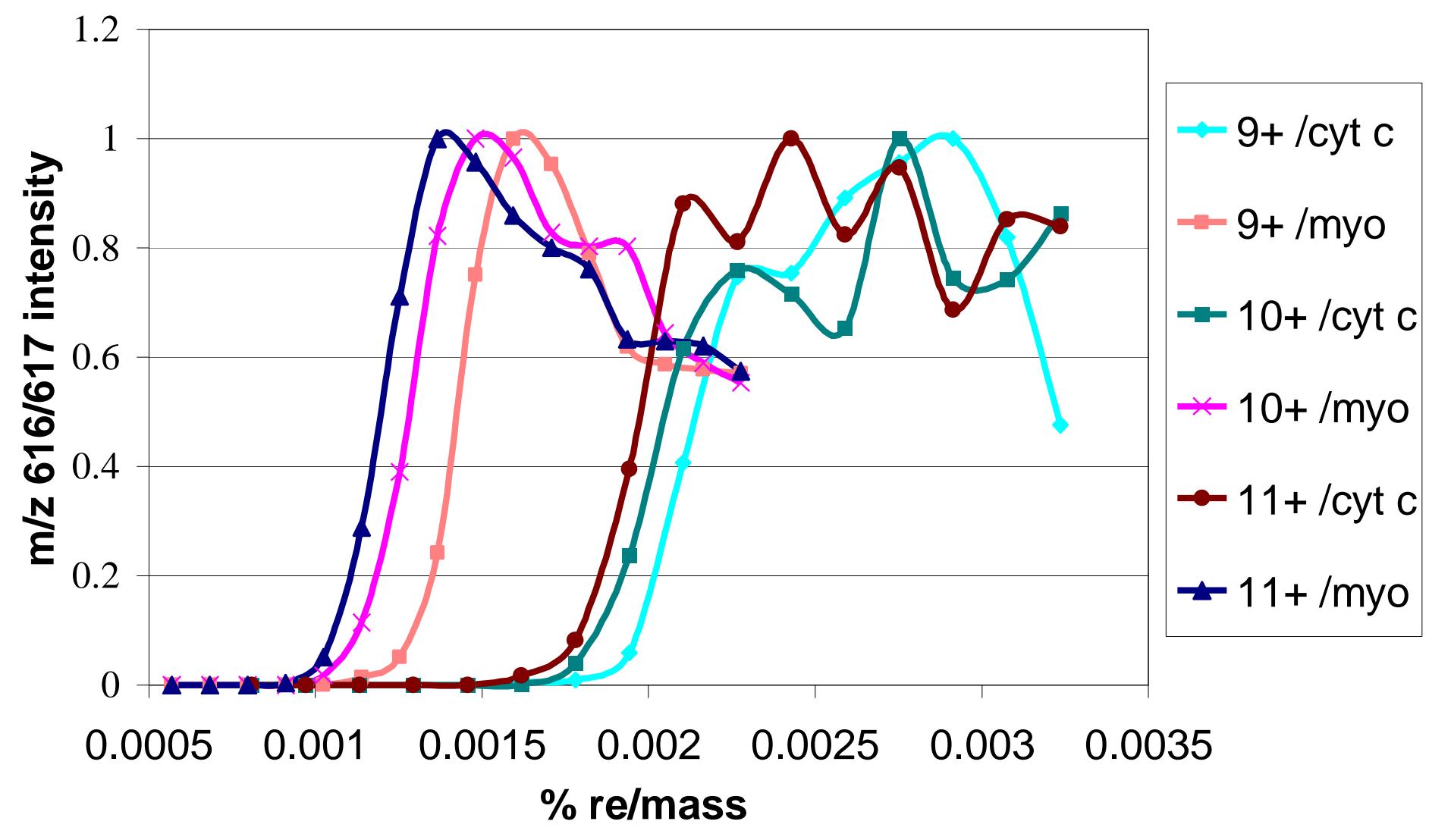

Figure 5.3.3: Heme Ion Intensity versus Relative Collision Energy for the 9+ to 11+ Charge States of Myoglobin and Cytochrome c.

The mass-corrected plots of the like charge states of myoglobin and cytochrome c clearly show the difference in the energy required to liberate the heme group from two proteins. In myoglobin, the prosthetic heme group is not covalently attached, but is merely ligated to the protein to the iron center at the core of the heme group and held by hydrogen bonds and van der Waals forces. It is therefore more readily removed by collision than from cytochrome c, wherein the two covalent bonds between the terminal vinyl groups of the porphyrin ring and the Cys14 and Cys 17 residues of the protein tether the porphyrin to the protein, in addition to weak intermolecular and inter-atomic attractions. These covalent linkages serve to stabilize the heme- 
protein complex. Thus for the like charge states more energy per unit mass is required to liberate the heme group from cytochrome $\mathrm{c}$ than is required to dissociate the same group from myoglobin

\subsection{Conclusions}

The reduced energy demand to liberate the heme group by CID from the higher charge states is consistent with the earlier findings of Hunter ${ }^{51}$. Their work on myoglobin, obtained by performing CID on quadrupole instruments in order to observe the ratio of the heme ion to the apoprotein that results upon the dissociation of heme proteins, did not extend to heme proteins such as cytochrome $\mathrm{c}$ wherein the prosthetic group is covalently associated with the polypeptide portion of the molecule. Our results are also in agreement with CD measurements and HD experiments that indicate that higher charge states represent more unfolded forms of a protein. The difference in energy to liberate the heme group for like charge states of the two proteins observed in the mass-corrected heme dissociation plots (Figure 5.3.3) would appear to reflect the strength of the heme-protein association. The greater energy demand to dissociate the heme group from cytochrome $\mathrm{c}$ can be attributed to the presence of the two thioether bonds that link the heme group to the protein. These covalent linkages are absent in myoglobin, and thus the prosthetic heme is more readily dislodged from the structure by CID. 


\section{Chapter 6: The Dependence of the Observed Fragmentation Pattern on the Oxidation State of the Heme Iron in the CID of Heme Proteins}

\subsection{Introduction}

It is known from many sources ${ }^{12,32}$ that as a result of MS/MS of heme proteins, the heme unit is liberated as a fragment ion. In the case of myoglobin, the heme unit is observed at $\mathrm{m} / \mathrm{z} 616$; in cytochrome $\mathrm{c}$ the heme fragment is observed at $\mathrm{m} / \mathrm{z} 617$. This is of interest because the two proteins possess the same heme type (ferroprotoporphyrin IX), and the metals are thought to be observed in the same oxidation state. In this work, we seek to provide information about the fragmentation of the heme unit from these proteins by performing multiple stages of MS, and propose a theory as to why the two ions prefer to follow different fragmentation pathways.

Heme proteins are an important class of macromolecules that play key roles in many significant biological processes. They are characterized by the presence of a porphyrin ring and one to several protein subunits. The iron atom at the center of the porphyrin may be found in the +2 or +3 oxidation state. This imparts abilities to the protein to serve as a center for electron transfer (cytochrome c), to participate in oxidation-state dependent reversible oxygen binding (hemoglobin and myoglobin), and to regulate biosynthesis and drug metabolism (cytochrome P450). The redox capabilities of heme proteins therefore equip the molecule, and thus afford the cell, with unique properties that allow the heme proteins to participate in many functions that are vital to cellular function and vitality. Figure 6.1.1 shows the structure of ferroprotoporphyrin IX, the prosthetic heme group found in hemoglobin, myoglobin, and cytochrome $\mathrm{c}$. 


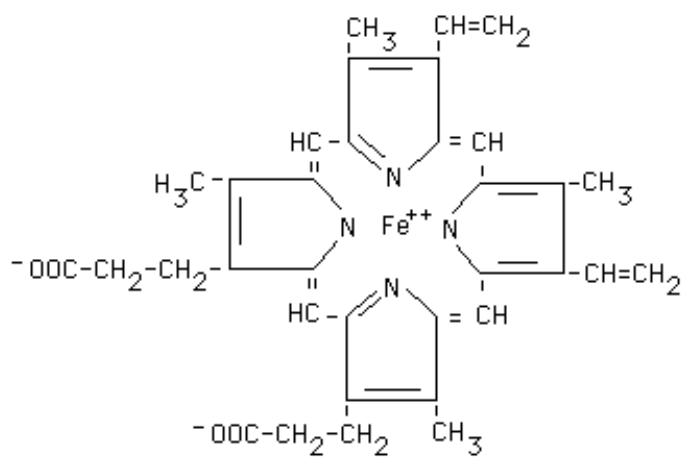

Figure 6.1.1: Structure of Ferroprotoporphyrin $\mathrm{IX}^{30}$.

Cytochrome $\mathrm{c}$ is a $12 \mathrm{kDa}$ heme protein that is found is bacterial mitochondrial membranes. It resides within the mitochondrial wall where it may interact with numerous redox centers to shuttle electrons from center to center in order to facilitate metabolic reactions within the cell. In this protein, the heme unit is close to the surface of the protein. Thus the iron molecule at the center of the porphyrin structure is accessible to electron transfer. Cytochrome $\mathrm{c}$ plays a central role in mitochondrial respiration in bacterial cells, catalyzing electron transfer reactions within the organelle that are responsible for energy production within the cell.

In cytochrome c, coordination about the metal center is octahedral, with four coordination sites occupied by the porphyrin skeleton. The two remaining sites are occupied by histidine and methionine residues from the protein. Figure 6.1.1 shows the coordination of the central iron atom in cytochrome c. 


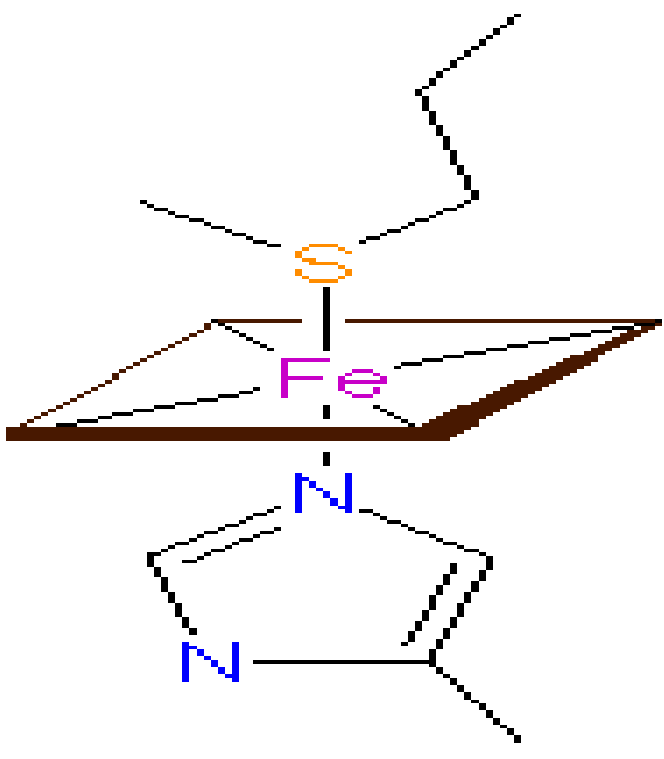

Figure 6.1.2: Coordination About the Central Iron Atom in Cytochrome $\mathrm{c}^{30}$.

In addition to the coordination sites, the porphyrin is connected to the protein by two cysteine bridges. As a post-translational modification, two thioether bonds are formed between the terminal acidic groups of the porphyrin and two cysteine sulfur groups of the protein subunit. This arrangement imparts stability of position to the heme unit within the structure, and anchors the porphyrin at the molecular surface where it can be readily oxidized or reduced.

In another group of heme-containing proteins, the globins, the coordination about the central iron atom in the porphyrin differs from that found in the cytochromes. In the globin class of heme proteins which contains myoglobin and hemoglobin, the arrangement about the iron center is 5-coordinate. As in the cytochromes, an axial coordination site is occupied by a histidine residue of the protein, and the equatorial sites are coordinated to the central portion of the porphyrin core. Figure 2-5 shows the coordination about the iron atom in myoglobin. 


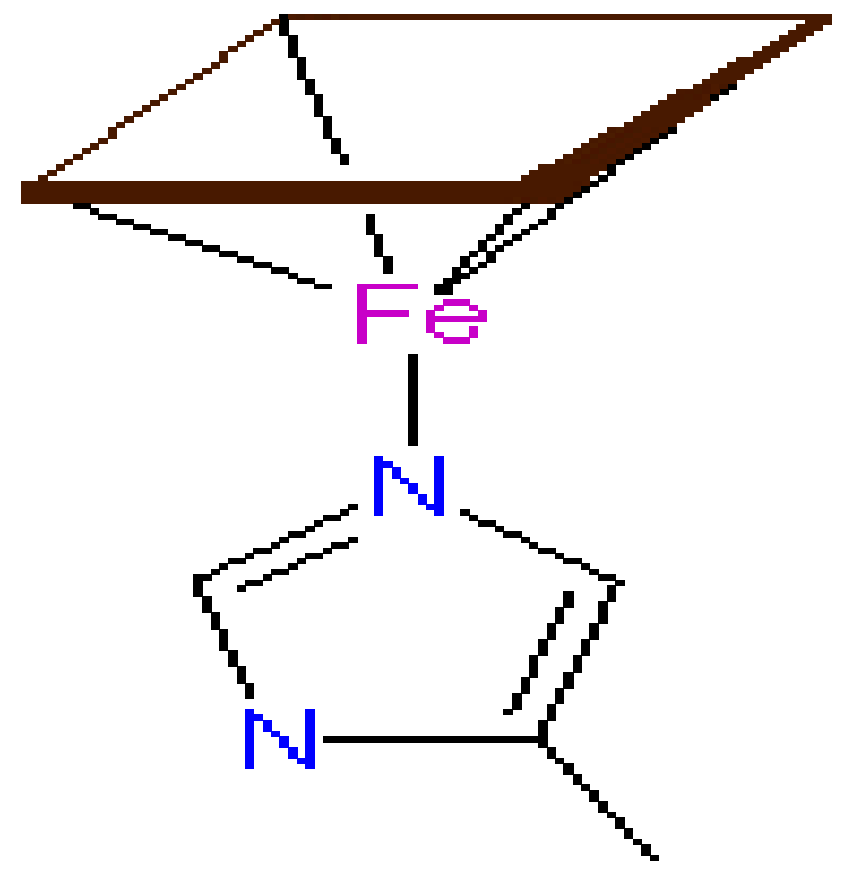

Figure 6.1.3: Coordination About the Heme Iron in Myoglobin ${ }^{30}$.

However unlike in the cytochromes, the remaining axial site is unoccupied in the globin heme proteins. This axial site is used to reversibly bind oxygen, thus these proteins function in the transportation and storage of molecular oxygen.

When the iron is in the oxidized state, the d5 electron configuration affords a vacant axial site, as the configuration about the metal center is square pyramidal. Thus when the molecule is in the +3 oxidation state, the oxygen binds to the available coordination site. Upon reduction, the oxygen is released and the cycle is repeated. Figure 6.1.3 shows a schematic of the binding process. 

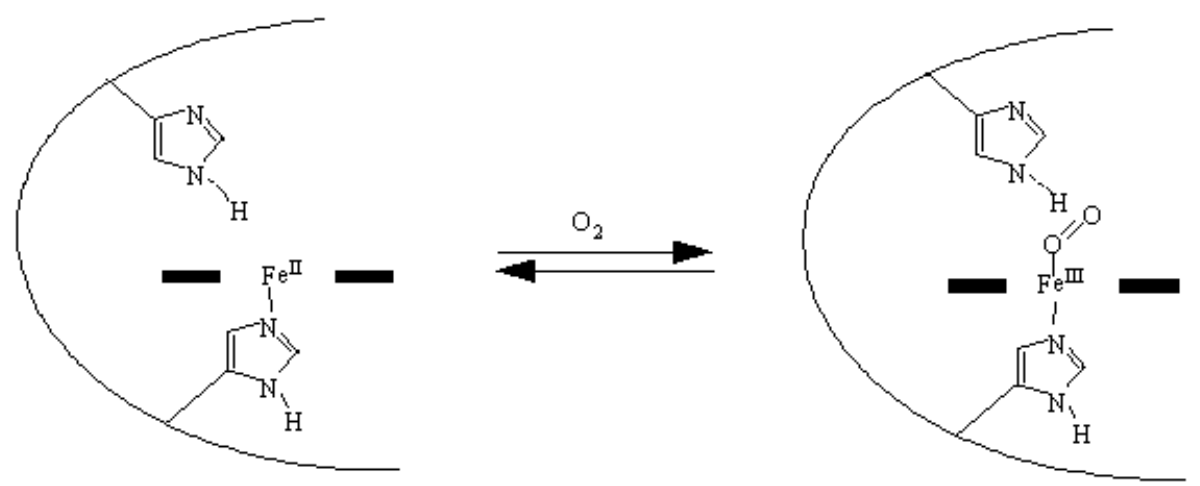

Figure 6.1.4: Oxygen Binding in Myoglobin ${ }^{30}$.

As in cytochrome $\mathrm{c}$, the porphyrin is held near the molecular surface and thus the iron center is accessible for oxygen binding and release. But unlike the cytochromes, the globin protein is not covalently linked to the porphyrin. The prosthetic heme is secured by a series of weak interactions (hydrogen bonding, non-polar, van der Waals).

\subsection{Materials and Methods}

Horse skeletal myoglobin and bovine cytochrome c were obtained from Sigma, and were used without purification. Solutions of the proteins were prepared in deionized water at concentrations of $125 \mu \mathrm{g} / \mathrm{ml}$ (myoglobin) and $100 \mu \mathrm{g} / \mathrm{ml}$ (cytochrome c). Spectra were acquired on an ion trap mass spectrometer (Finnigan LCQ) fitted with a standard electrospray ion source. Samples were infused to the ion source by syringe pump at a flow rate of $3 \mu 1 / \mathrm{min}$.

Instrument conditions were optimized in order to observe the maximum number of charge states of holoprotein while exhibiting sufficient sensitivity to perform the desired analyses. Once established, the same conditions were used for the analysis of all charge states of both proteins. Thus all of the data shown were obtained under identical conditions so that direct comparisons could be made. 
In order to evaluate heme dissociation from the holoproteins, a full spectrum of the protein was first acquired. For each charge state, a 3 AMU window was constructed around the most abundant mass to isolate the selected charge state.

\subsection{Results}

\section{Cytochrome c}

Figure 6.3.1 shows the full spectrum of cytochrome c. Note that the heme unit is not observed, as CID is required in order to liberate the heme group from the structure.

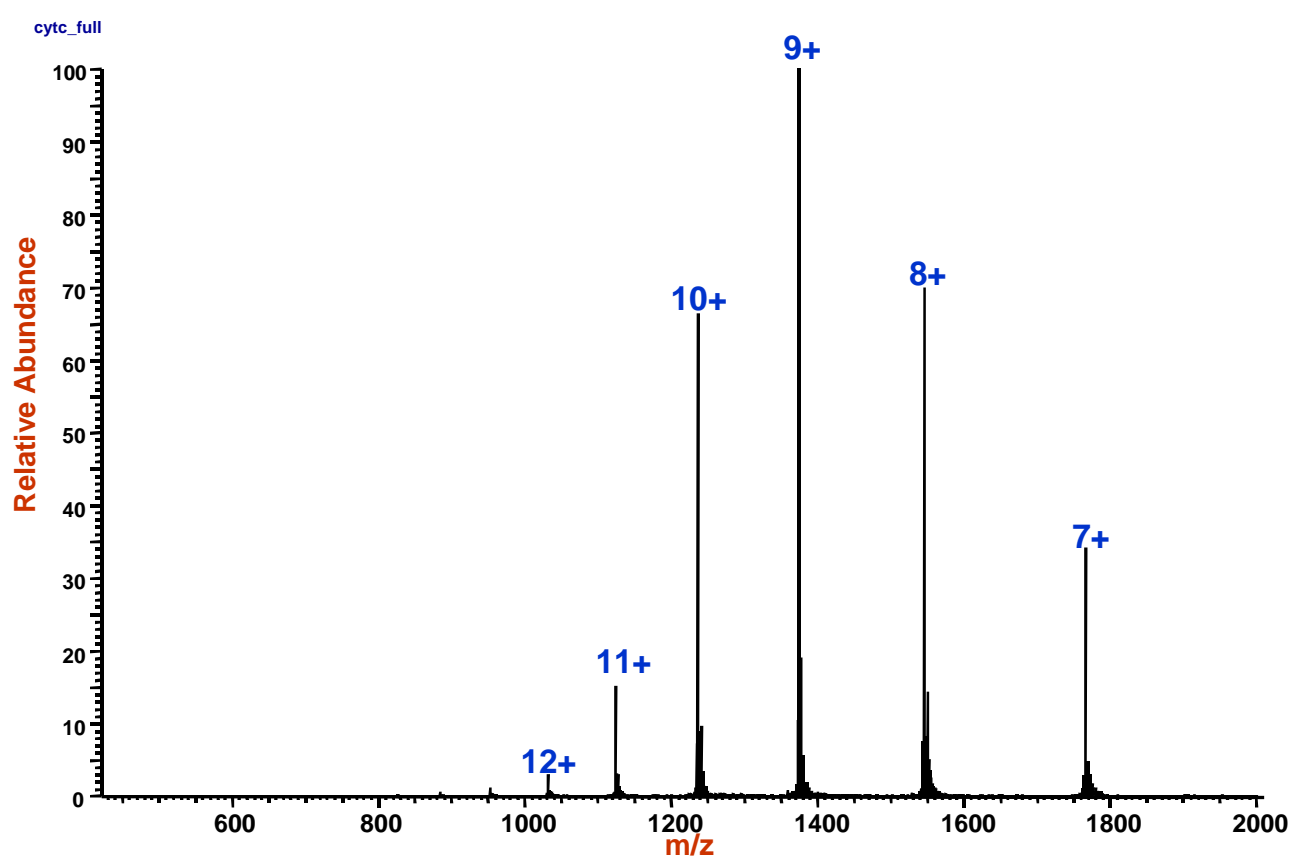

Figure 6.3.1: Mass Spectrum of Cytochrome c 
When the above 9+ charge state is subjected to MS/MS, the heme unit is observed as a singly charged species at $\mathrm{m} / \mathrm{z}$ 617. Figure 6.3.2 shows the MS2 spectrum of the 9+chargestate of cytochrome c.

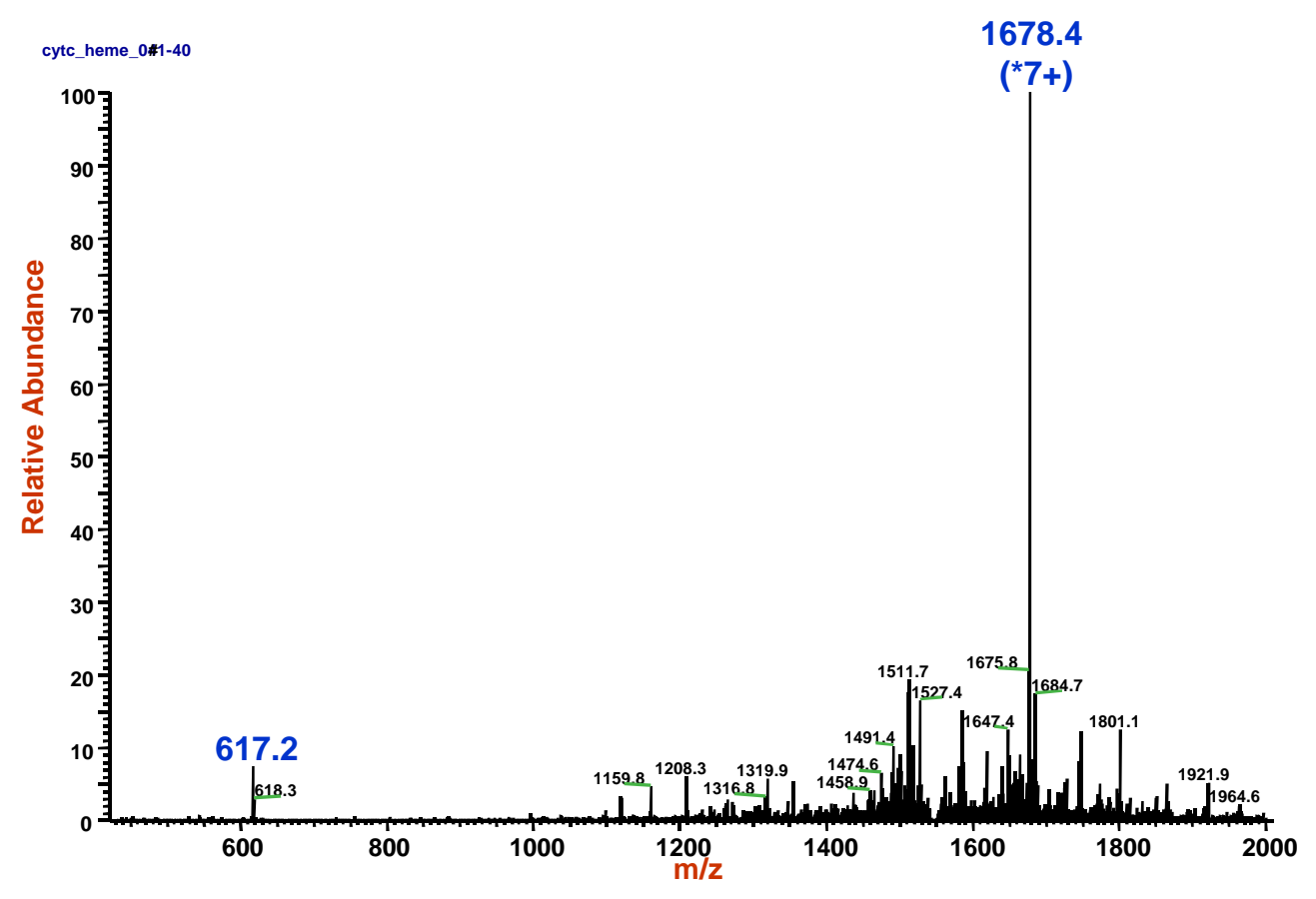

Figure 6.3.2: MS2 Spectrum of the 9+ Charge State of Cytochrome c.

An additional stage of MS on the 8+/617 masses yields the spectrum shown in Figure 6.3.3, indicating that the fragment ions of 617 are observed at $\mathrm{m} / \mathrm{z} 544,557$, and 602 . 


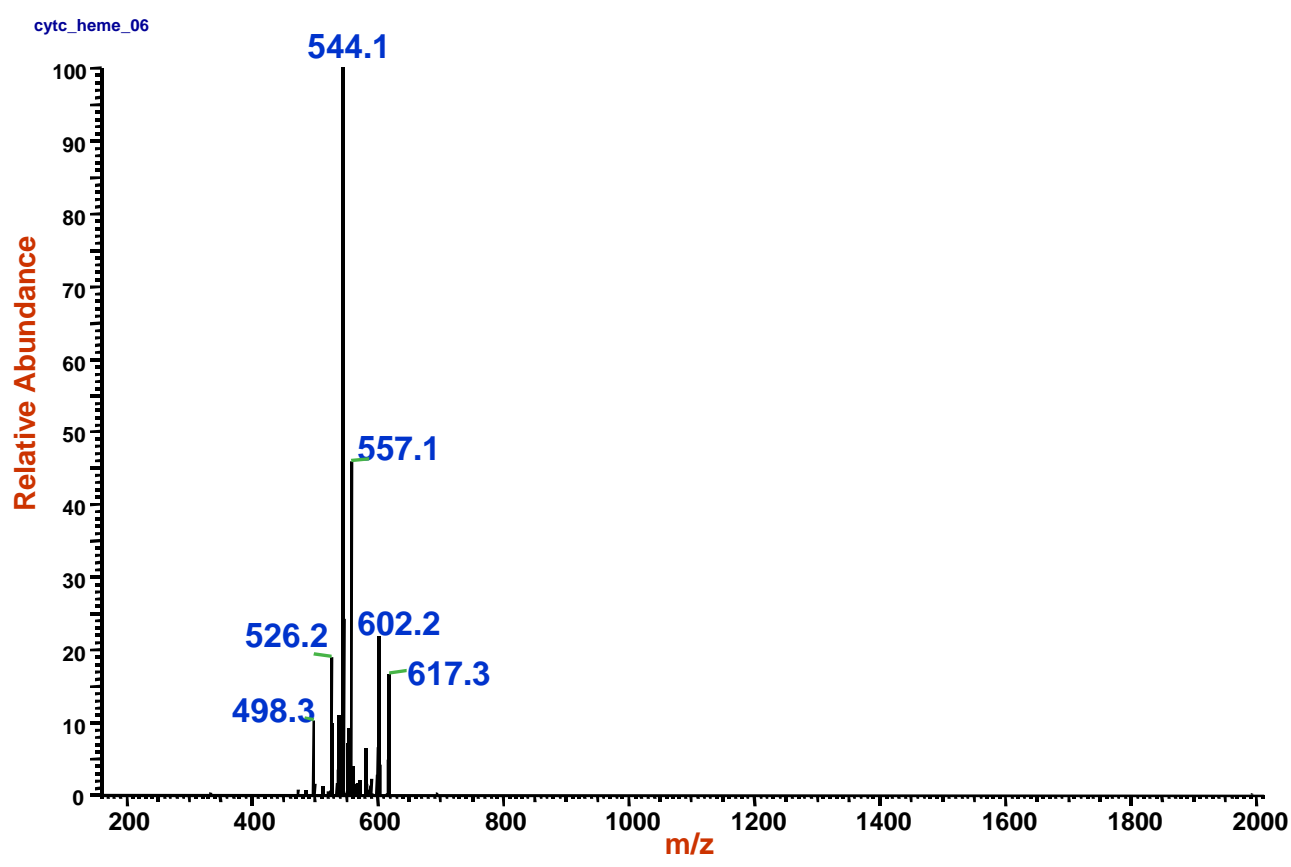

Figure 6.3.3: MS3 Spectrum of m/z $1374(9+) / 617$ for Cytochrome c.

In subjecting the 544 mass fragment to an additional stage of MS, the spectrum shown in Figure 6.3.4 is obtained. The observed base peak is at $\mathrm{m} / \mathrm{z} 485$, with minor fragments at $\mathrm{m} / \mathrm{z} 486$,

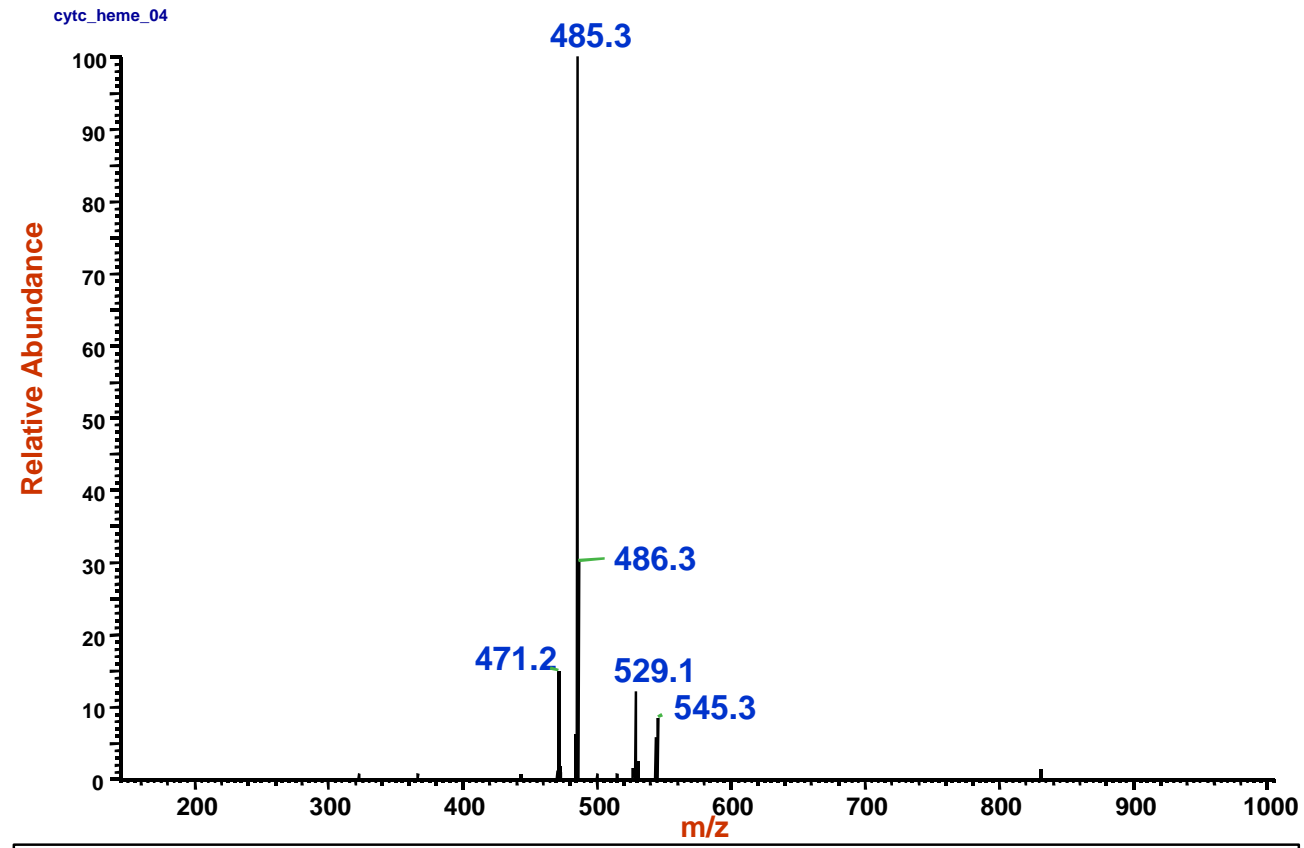

Figure 6.3.4: MS4 Spectrum of 8+/617/544 for Cytochrome c. 
471, and 529.

When the fragment of $\mathrm{m} / \mathrm{z} 617$ at $\mathrm{m} / \mathrm{z} 557$ is subjected to CID, the spectrum shown in Figure 6.3.5 is obtained. The base peak is observed at $\mathrm{m} / \mathrm{z} 498$, with fragments of lesser abundance at $\mathrm{m} / \mathrm{z}$ values of $499,543,485$, and 539 .

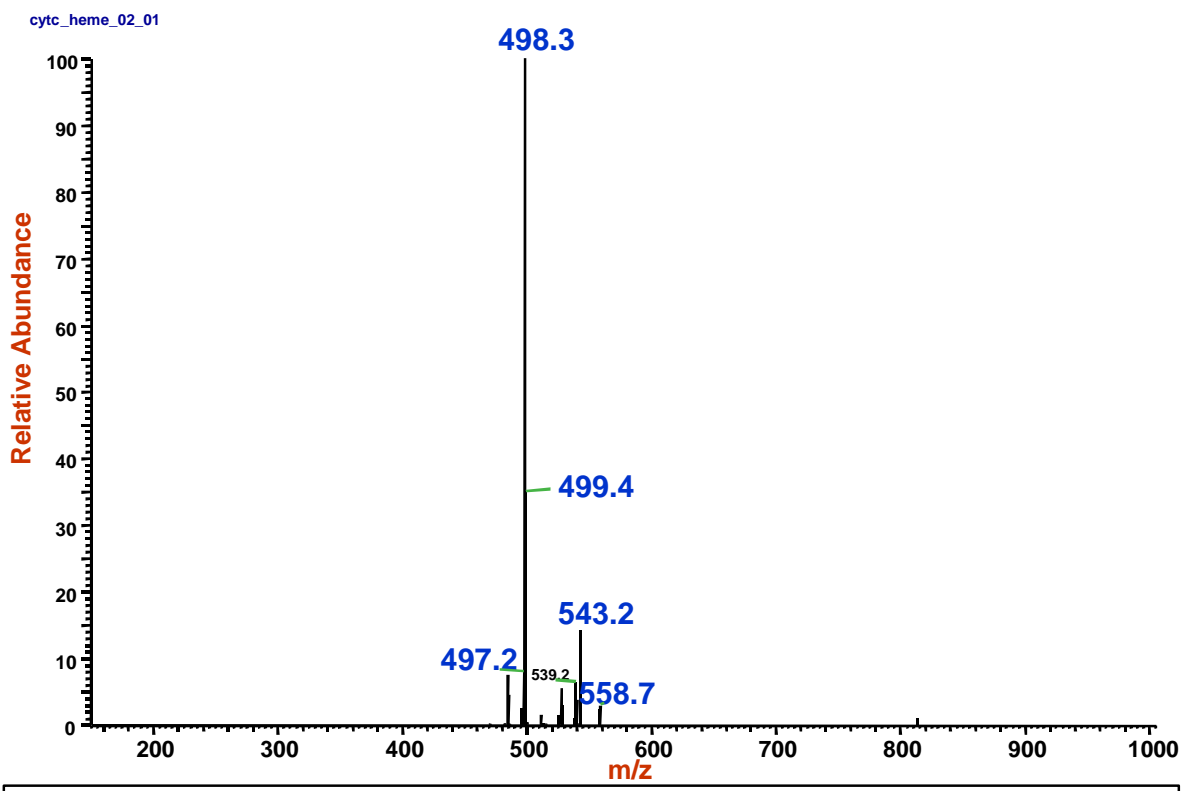

Figure 6.3.5: MS4 Spectrum of 9+/617/557 for Cytochrome c. 


\section{Myoglobin}

As previously noted, the heme unit as liberated by CID from myoglobin is observed at $\mathrm{m} / \mathrm{z} 616$.

Figure 6.3.6 shows the full mass spectrum of myoglobin in deionized water. As the heme group is not covalently linked to the protein backbone as it is in cytochrome $\mathrm{c}$, the prosthetic group is more easily dislodged from the structure. Thus as the heme group was not observed in the full MS of cytochrome c, Figure 6.3.6 illustrates that it is evident at m/z 616 in the full mass spectrum of myoglobin. Loss of the heme unit from the holoprotein results in the formation of the corresponding apoprotein., thus a doublet of peaks corresponding to the apoprotein and the holoprotein are present in the spectrum for each charge state.

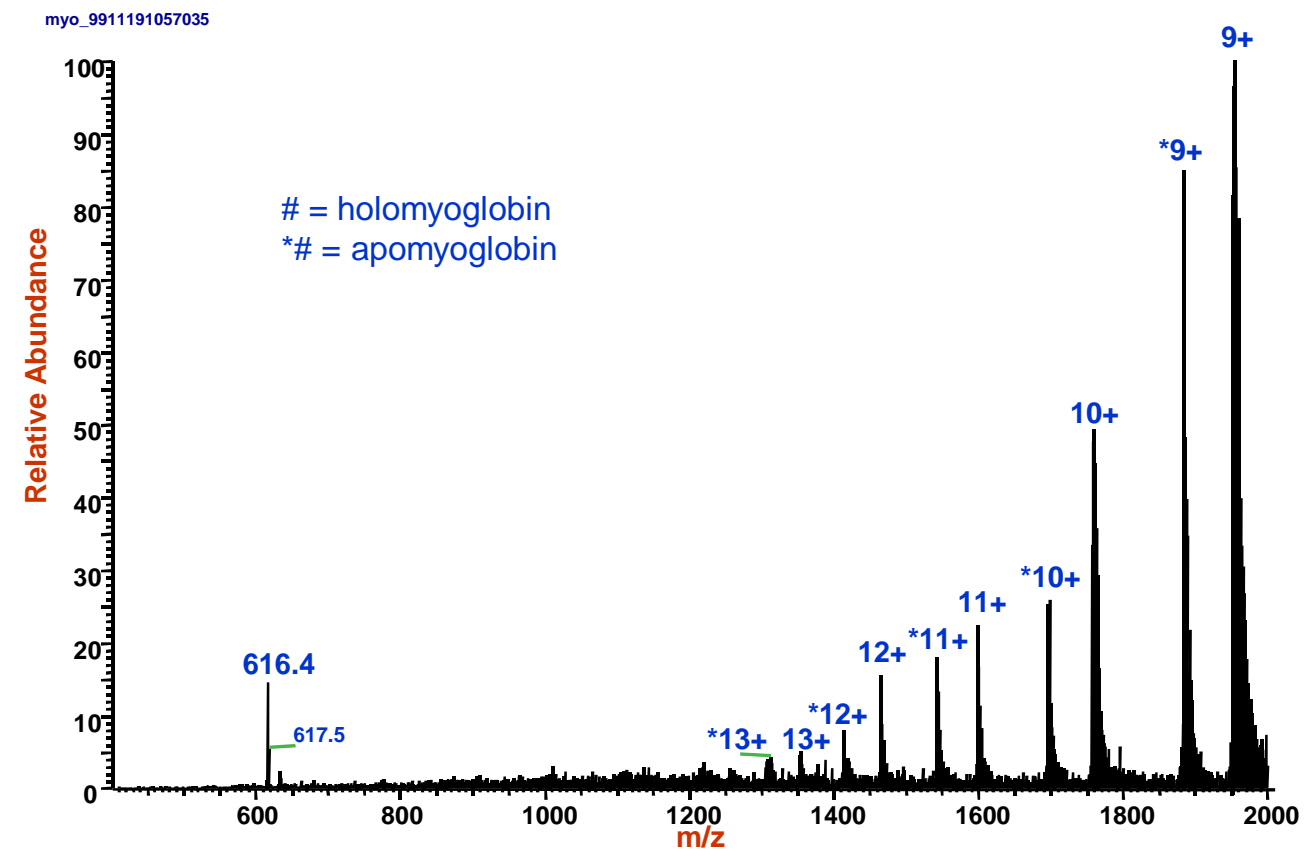

Figure 6.3.6: Full Mass Spectrum of Myoglobin 
MS/MS analysis of the 9+ charge state of holomyoglobin results in the liberation of the heme unit as observed at $\mathrm{m} / \mathrm{z} 616$, and in the formation of the $9+$ charge state of apomyoglobin as shown in Figure 6.3.7.

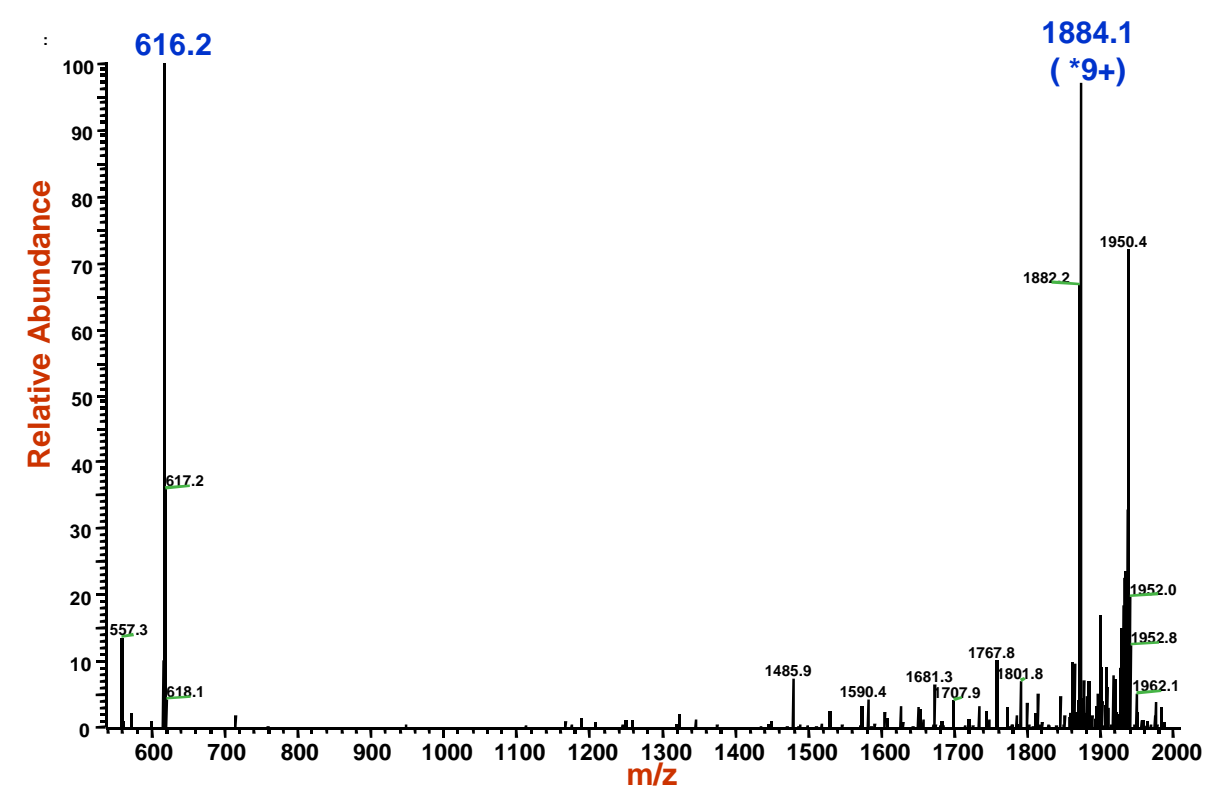

Figure 6.3.7: MS/MS of the 9+ Charge State of Holomyoglobin

MS3 analysis of 9+/616 results in the spectrum shown in Figure 6.3.8, where fragment ions of the heme group are observed at m/z 557, 558 and 544.

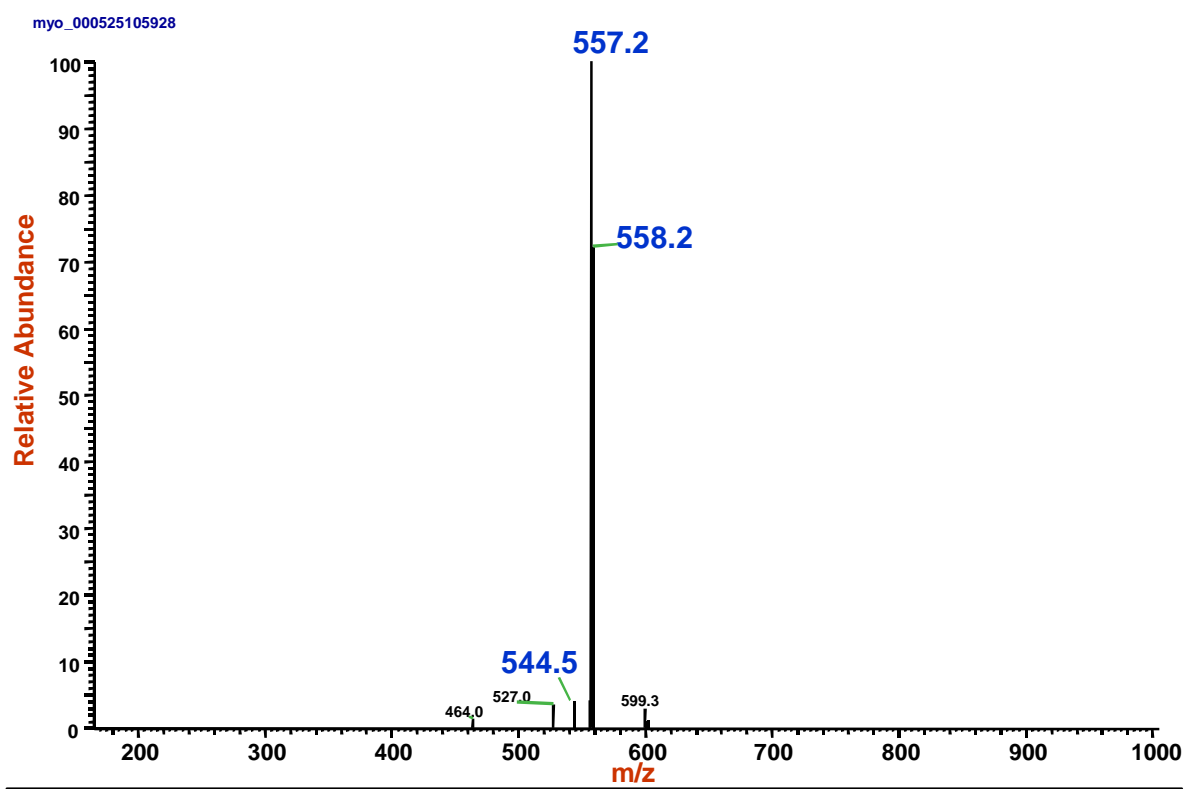


Taking the analysis a further step, the MS4 spectrum of $9+/ 616 / 557$ is shown in Figure 6.3.9. Here the most intense peak is observed at $\mathrm{m} / \mathrm{z} 498$, with minor fragments at $\mathrm{m} / \mathrm{z}$ values of 499, 539, and 485 .

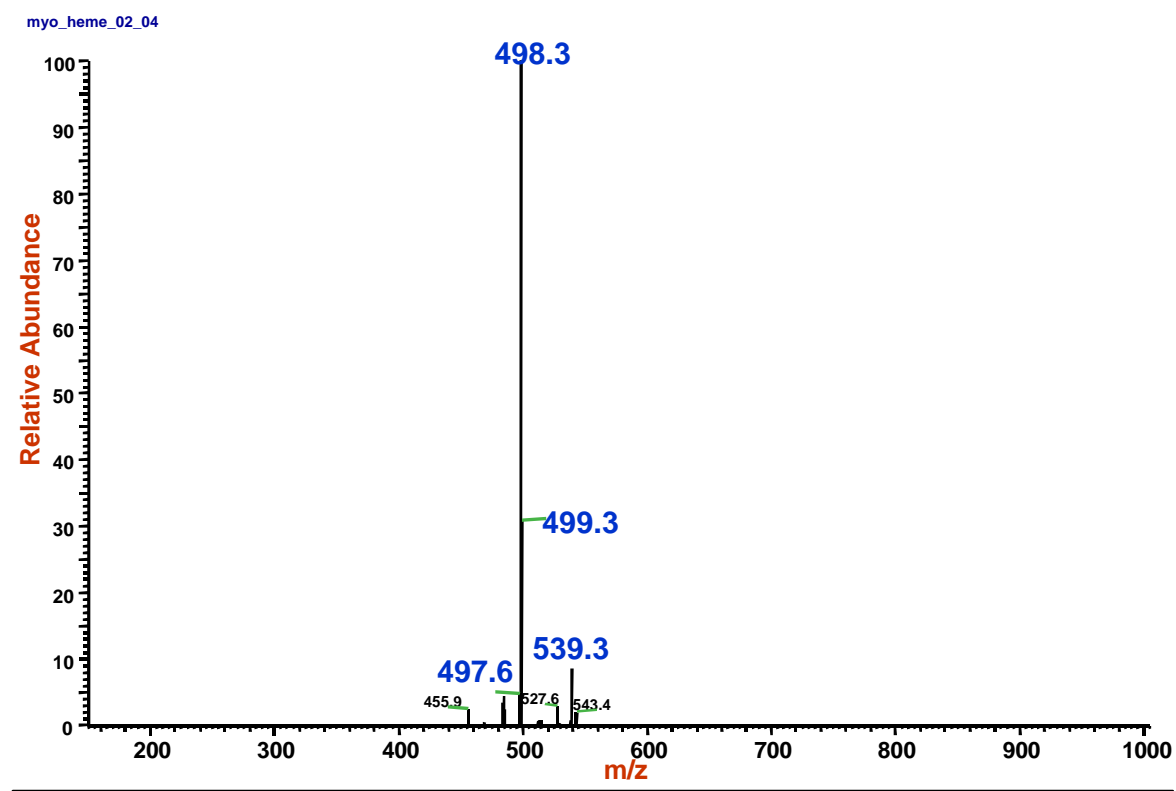

Figure 6.3.9: MS4 of 9+/616/557 for Myoglobin.

When the minor fragment from the MS3 analysis at $\mathrm{m} / \mathrm{z} 544$ is subjected to CID, the spectrum shown in Figure 6.3.10 is obtained. Here the heme fragment at m/z 557 is seen to give $\mathrm{m} / \mathrm{z} 485$ as the most abundant fragment, with other fragments appearing at $\mathrm{m} / \mathrm{z} 484,499,528$, and 470. Due to the low abundance of the $\mathrm{m} / \mathrm{z} 544$ fragment as a result of the CID of $\mathrm{m} / \mathrm{z} 616$, the spectrum shown below was observed at very low signal abundance. 


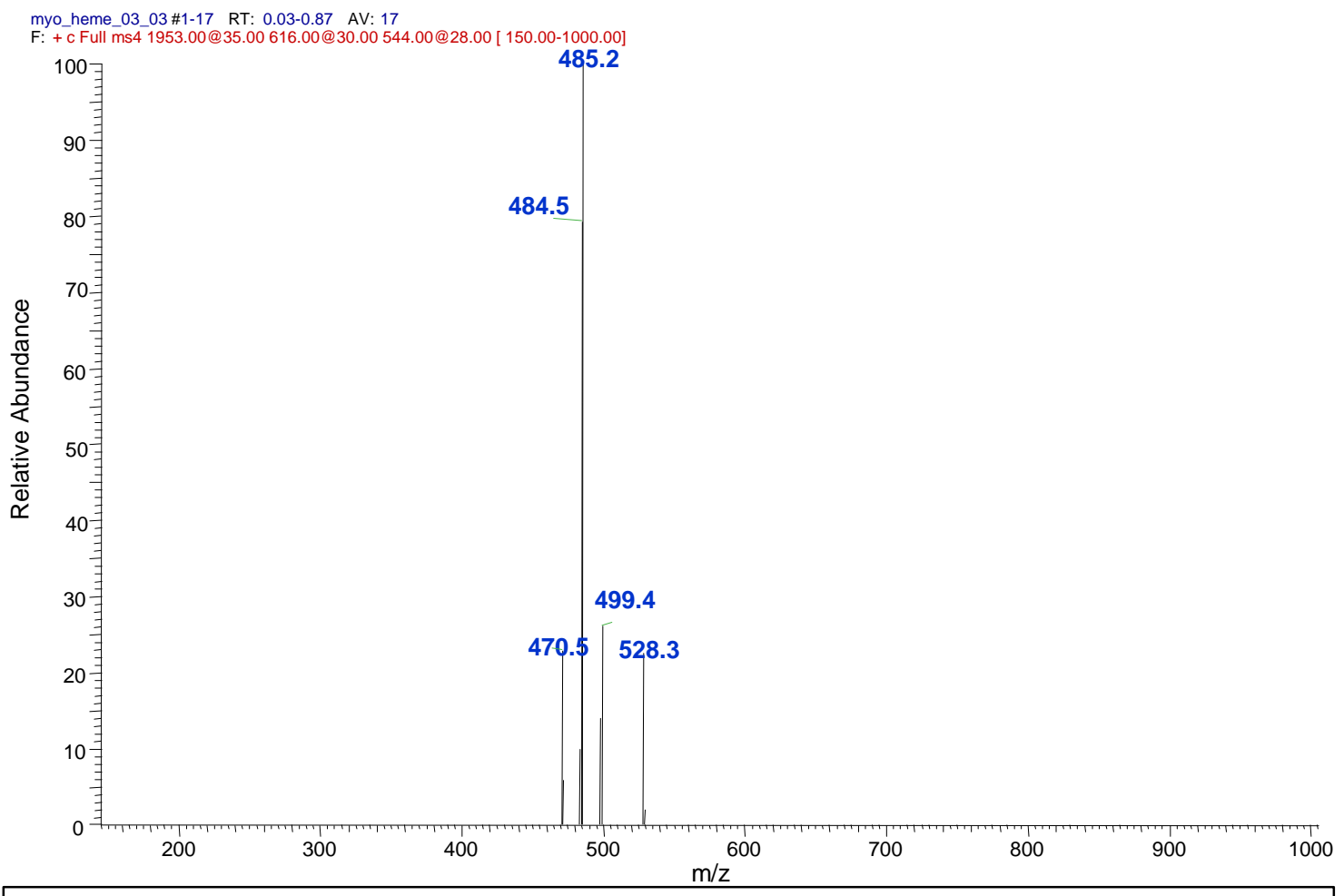

Figure 6.3.10: MS4 of 9+/616/544 for Myoglobin. 


\subsection{Discussion}

Analysis of the spectra for the two proteins reveals that while the mass of the heme group evolved from the two proteins differs by one atomic mass unit, the fragment spectra of the prosthetic groups liberated from the two proteins share some similarities. 6.4.1 summarizes the observed fragment ions derived from the heme groups of cytochrome $\mathrm{c}$ and myoglobin.

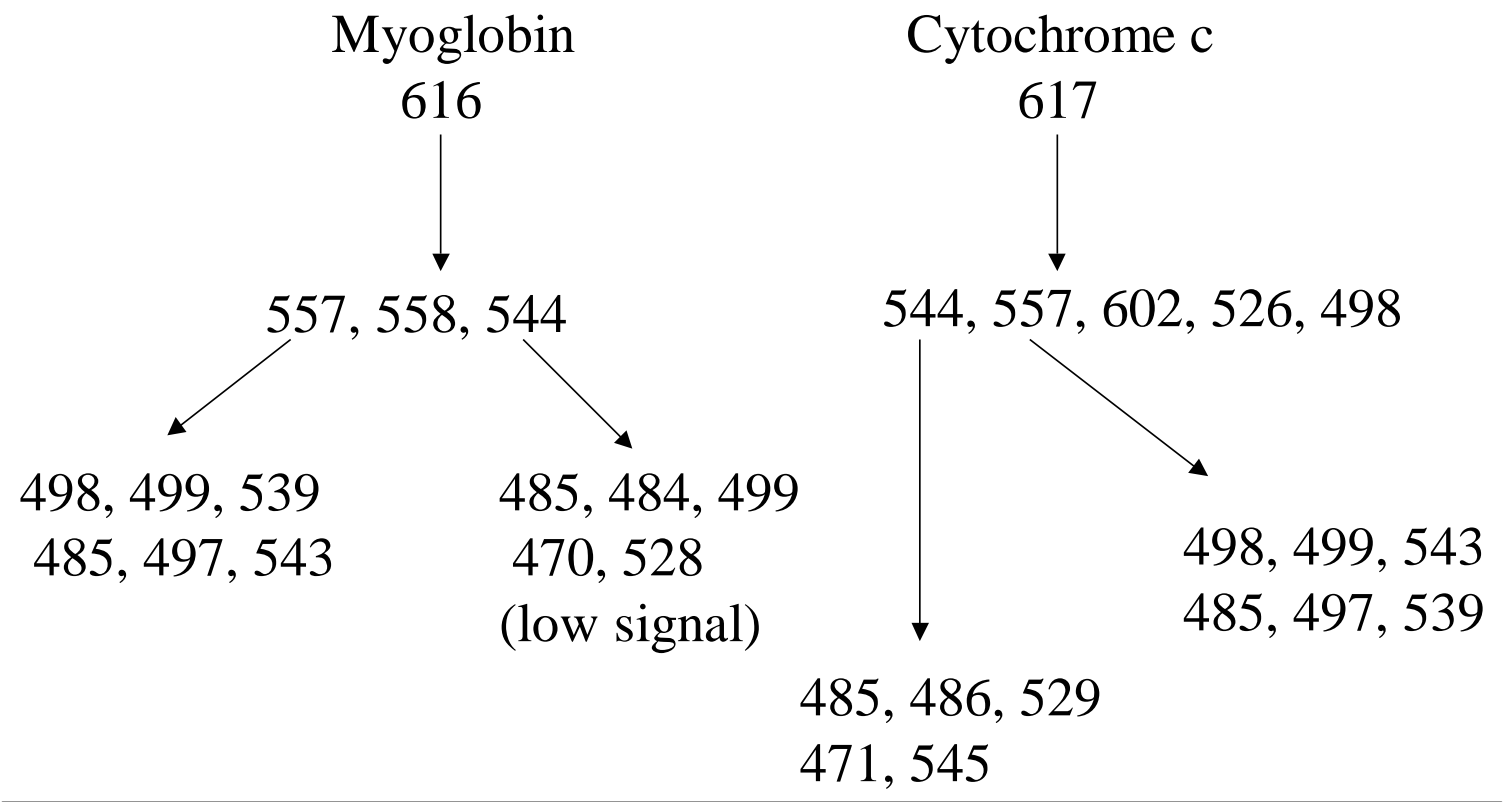

Figure 6.4.1: Observed Fragmentation of the Heme Ions from Myoglobin and Cytochrome c.

As is illustrated in the figure above, the difference in the fragmentation pattern of the heme groups derived from the two proteins is not in the masses of the ions observed, but in the relative abundance of those ions. In the initial stage of fragmentation, the heme group from cytochrome c degrades to $\mathrm{m} / \mathrm{z} 544$ as the base peak, with $\mathrm{m} / \mathrm{z} 557$ at $40 \%$ relative abundance. However when the heme group from myoglobin at $\mathrm{m} / \mathrm{z} 616$ is subjected to CID, the most abundant fragment ion is found at $\mathrm{m} / \mathrm{z} 557$; the mass fragment at $\mathrm{m} / \mathrm{z} 544$ is observed at only $7 \%$ 
relative abundance. Therefore the same masses are observed as fragment ions of the heme groups liberated from the two proteins, but they are observed at different relative abundances.

The data shown demonstrates that the fragment ions of $617 / 616$ at $\mathrm{m} / \mathrm{z} 557$ and at $\mathrm{m} / \mathrm{z}$ follow the same fragmentation pathways when subjected to an additional stage of CID. The minor fragment of the heme group from cytochrome $\mathrm{c}$ at m/z 557 upon CID decomposes to form ions of masses 498, 499, 543, 485, 497, and539, CID of the major heme fragment from myoglobin at m/z 557 yields a virtually identical spectrum. Figure 6.4.2 shows a comparison of the spectra of the fragment ions observed upon the dissociation of the $\mathrm{m} / \mathrm{z} 557$ ion generated from the heme group of both proteins. Additionally, when the heme fragment from cytochrome c at $\mathrm{m} / \mathrm{z} 544$ is subjected to MS/MS, fragment ions appear at $\mathrm{m} / \mathrm{z}$ values of $485,486,529,471$, and 545. MS/MS analysis of the minor heme fragment at $\mathrm{m} / \mathrm{z} 544$ from myoglobin results in the observation of the similar masses, though the spectrum was obtained at low signal intensity. Figure 6.4.3 shows a comparison of the CID of $\mathrm{m} / \mathrm{z} 544$ spectra for cytochrome $\mathrm{c}$ and myoglobin. 


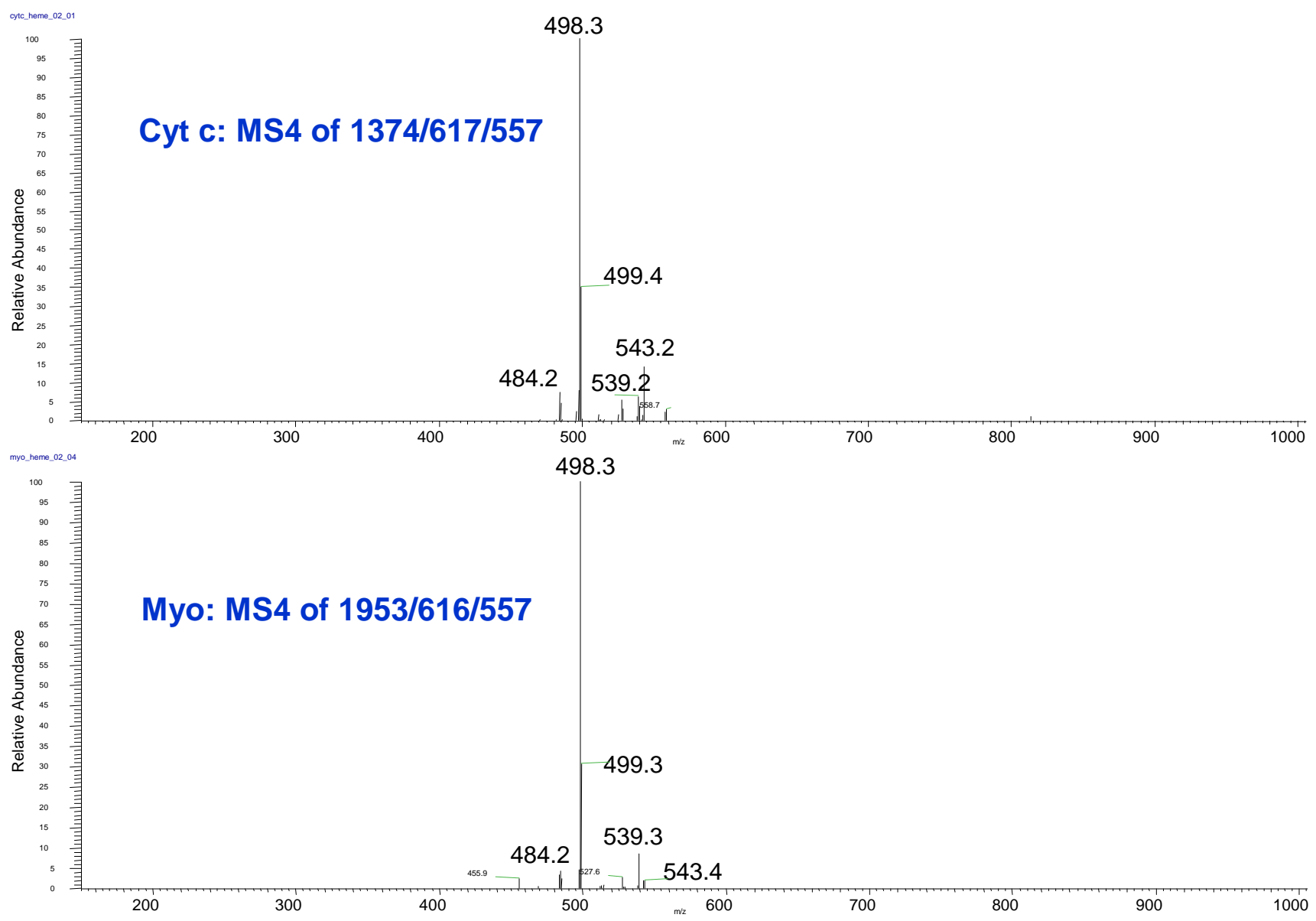

Figure 6.4.2: Comparison of MS4 of m/z 557 Spectra for Cytochrome $\mathrm{c}$ and Myoglobin. 

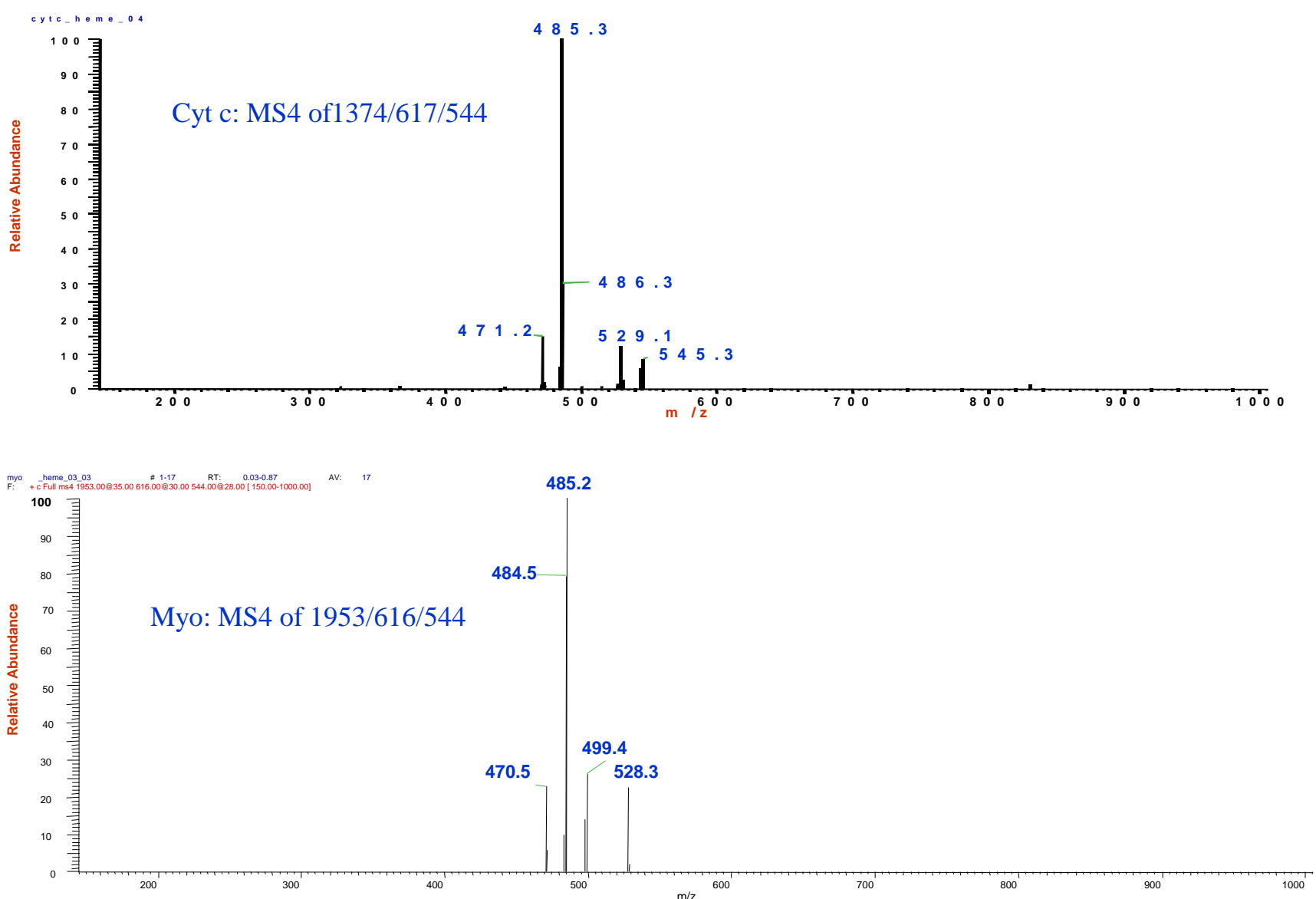

Figure 6.4.3: Comparison of the MS4 of 544 Spectra for Cytochrome $c$ and Myoglobin. 
It is the clear that the fragment ions resulting from CID of the heme groups from the two proteins are observed at the same masses, and the fragment ions of the heme groups from the two proteins follow the same fragmentation pathways. The difference in masses of the heme groups, $\mathrm{m} / \mathrm{z} 617$ from cytochrome $\mathrm{c}$ and $\mathrm{m} / \mathrm{z} 616$ from myoglobin, results in a bias toward one of the two observed fragmentation pathways. In cytochrome $\mathrm{c}$, where the heme group is observed at $\mathrm{m} / \mathrm{z}$ 617, the 544/485 pathway is preferred, while in myoglobin CID of the heme group at $\mathrm{m} / \mathrm{z} 616$ suggests that the 557/498 pathway is the preferential pattern of dissociation.

The difference in mass, then, must suggest a structural difference between the two heme ions that causes one fragmentation pathway to be preferred in one species that is disfavored in the other. Recent ICR experiments by Marshall's group ${ }^{32}$ may shed some light on the situation. Their work suggests that the metal center in both proteins is found in the +3 oxidation state, and that upon CID the iron is reduced to +2 in cytochrome as a consequence of dissociation, yet remains in the +3 state in myoglobin. Thus the difference in mass of the heme ions from myoglobin and cytochrome $\mathrm{c}$ can be attributed to the oxidation state of the iron atom at the core of the heme group. This indicates that the heme ion from myoglobin can most appropriately be described as $\left[\mathrm{Fe}^{+3}+\text { heme }\right]^{+}$, while the heme ion from cytochrome $\mathrm{c}$ is of the form $\left[\mathrm{Fe}^{+2}+\text { heme }+\mathrm{H}^{+}\right]^{+}$. The difference in the oxidation state of the iron and the presence of an additional proton in the heme derived from cytochrome then accounts for the observed mass difference of 1 AMU.

These oxidative and structural differences between the two ions must then be related to the preference of the heme derived from cytochrome c to follow the $\{$ heme-544-485\} pathway, while the $\{$ heme-557-498\} pathway is preferred for the heme group derived from myoglobin. 
Additionally, if the oxidation state of the metal were different, ions of the same mass as a result of CID would not be observed as is seen in this work. It seems then that in order for the two pathways to be equivalent, either the heme ion from cytochrome $\mathrm{c}$ is re-oxidized upon further CID, or the heme ion from myoglobin is reduced. Otherwise, the two fragmentation pathways would not overlap, resulting in equivalent MS4 spectra.

The fact that the $617 / 557$ pathway is represented to a very minor degree in cytochrome $\mathrm{c}$ may be telling. In order for the fragmentation to be equivalent, and assuming that myoglobin's heme does not change oxidation state, the heme group from cytochrome $\mathrm{c}$ would be required to lose an electron and a proton upon CID. This would return the metal to the +3 oxidation state, and by losing a proton during fragmentation the remaining heme ion could be equivalent to that derived from myoglobin.

It also may be that the heme iron in myoglobin is reduced upon CID. As the configuration about the metal center in the heme is square pyramidal, a vacant coordination site is present that may facilitate electron transfer to the heme iron. This site, no longer protected by the globin protein after the initial stage of CID, may allow reduction of the heme iron to occur during MS3 analysis. However again assuming that the heme iron in cytochrome c does not change oxidation state, the heme iron from myoglobin would be required to accept both an electron and a proton in order for the fragments of the two heme groups to be equivalent.

For cytochrome c, whose intact heme group is observed at $\mathrm{m} / \mathrm{z} 617$, to observe the ion at $\mathrm{m} / \mathrm{z} 544$ would require the loss of 73 mass units, corresponding to the loss of $\mathrm{CH}_{2}-\mathrm{CH}_{2}-\mathrm{COO}^{-}$ plus a proton. This loss would require that the metal center remain in the +2 oxidation state if the major fragment of m/z 544 is observed as a singly-charged species. This is the preferred mechanism in cytochrome $\mathrm{c}$, but this pathway is a low probability transition for the heme group 
from myoglobin. The disfavored pathway for the heme group from cytochrome $\mathrm{c}$ results in the ion of $\mathrm{m} / \mathrm{z} 557$, after a loss of 60 mass units form the heme group of $\mathrm{m} / \mathrm{z} 617$. This corresponds with the loss of $\left[\mathrm{CH}_{3}-\mathrm{COOH}\right]^{+}$. To be observed as a singly-charged ion, the metal center would be required to be oxidized to the +3 oxidation state, a low-probability process that accounts for the low relative abundance of the ion representative of this fragmentation pathway.

The ion at m/z 544 from myoglobin's heme represents the loss of 72 mass units from the intact porphyrin, or the loss in this case of $\left[\mathrm{CH}_{2}-\mathrm{CH}_{2}-\mathrm{COO}\right]$. This corresponds with the loss of a complete side chain from the porphyrin ring, lost as an anion. Therefore to be observed at $\mathrm{m} / \mathrm{z}$ 544, the metal center in myoglobin would be required to be reduced during the dissociative process. Given the complexity of this process, this would seem to be a low probability transition. The $\mathrm{m} / \mathrm{z} 544$ fragment is observed at only $5 \%$ abundance relative to the dominant process that results in the formation of $\mathrm{m} / \mathrm{z} 557$, and its observation at low relative abundance is consistent with a disfavored pathway.

The dominant process in myoglobin results in the formation of the ion of $\mathrm{m} / \mathrm{z} 557$ after the loss of 59 mass units from the heme ion of $\mathrm{m} / \mathrm{z} 616$. This represents the loss of a $\left[\mathrm{CH}_{2}\right.$ $\mathrm{COOH}$ group as a neutral species. This requires that a negative charge retained on the remaining carbon on the porphyrin ring, which is stabilized by conjugation to the ring system. The fragment may also be lost as an anion, but this would require that the metal be reduced to remain a singly charged ion.

The dominant processes of fragmentation for the heme groups from myoglobin and cytochrome $\mathrm{c}$ would appear to be those wherein no change to the oxidation state of the metal center is observed. The most abundant ions observed in the CID spectra are those that are derived from simple fragmentation of the heme group within the ion trap. Processes involving 
fragmentation of the heme along with changes to the oxidation state of the metal appear to be low-probability events. This assertion is supported by the observation that ions representative of such complex transitions appear in the CID spectra at relatively low abundance with respect to the ions that result from simple fragmentation of the heme group. Therefore the dominant pathways of fragmentation are those that involve no change to the oxidation state of the iron atom at the center of the porphyrin structure.

\subsection{Conclusions}

The 1 AMU difference in mass between the heme groups liberated by CID from myoglobin and cytochrome $\mathrm{c}$ is related to a difference in oxidation state of the metal at the center of the porphyrin structure. The iron atom at the center of the heme group is found in the +3 oxidation state in both intact proteins. The heme group from cytochrome $\mathrm{c}$ is reduced to the +2 oxidation state upon CID but remains in the +3 state when derived from myoglobin. The differences in mass and oxidation state cause the heme groups from the two sources to follow one of two competing fragmentation pathways. The pathway that is dominant in myoglobin is in evidence, but is disfavored for the heme group from cytochrome c. Conversely, the pathway that is disfavored in myoglobin is the dominant process for the heme group from cytochrome c.

Additionally, it appears that the favored processes represent simple fragmentation of the heme group without a change in oxidation state of the metal, while the disfavored processes require fragmentation of the heme group as well as a change to the oxidation state of the metal center. The processes requiring both fragmentation and a change of the oxidation state of the metal are more complex, lower probability transitions. This explains the observation that the ions 
representative of these complex transitions are observed at low abundance in the CID spectra of the heme groups derived from cytochrome $\mathrm{c}$ and myoglobin. 
Section 3: Electrospray lonization for the Analysis of Small Molecules 


\section{Chapter 7: Identification of a Metabolite of Nicotinylalanine using LC-ESI-MS}

\subsection{Introduction}

During development of new therapeutic drugs the metabolic fate of the drug candidate administered is an important consideration. The in-vivo formed metabolic products of new chemical entities being tested as drug candidates may possess biological activities and toxic properties that exceed those of the parent drug under evaluation as a new drug candidate.

Clearly, the identification of drug metabolites is of vital importance in the development of safe and effective pharmaceutical preparations. In recent years, liquid chromatography coupled with mass spectrometry (LC-MS) has played a significant role in the rapid screening of new drug candidates. The interfacing of electrospray ionization with ion trap mass spectrometry to provide tandem MS capabilities permits the ready structural elucidation of metabolites.

Nicotinylalanine (NAL, 2-amino-4-oxo-4-(pyridyl) butanoic acid) and kynurenic acid are structurally related to antagonists of central nervous system excitatory receptors. The enzymes involved in the metabolic pathway leading from tryptophan to kynurenic acid are inhibited by $\mathrm{NAL}^{56}$. In addition to acting at CNS receptors, nicotinylalanine is converted to nicotinamide in normal mammalian tissue. Decreased nicotinamide has been associated with virally infected cells and may disrupt postranslational poly (ADP) ribosylation reactions of viral proteins ${ }^{57} 58$ Intracellular nicotinamide levels in mice can be enhanced by administration of a combination of NAL and acetylsalicylic acid. This combination may prove useful as an anti-AIDS therapy ${ }^{59}$. In this communication, we report the identification of $\mathrm{N}$-acetylnicotinylalanine, a major urinary metabolite in mice of NAL other than nicotinamide. This less-polar metabolite, isolated by HPLC, reverted to NAL under hydrolytic conditions in acid. Identification of this metabolite of 
NAL was achieved through comparison with standard $N$-acetylnicotinylalanine synthesized in the laboratory. The urinary metabolite was characterized by LC retention time, single-stage mass spectrometric analysis, and multiple-stage mass spectrometric analysis. The LC retention time and mass information gathered for the urinary metabolite was then compared with the same information for synthetic $N$-acetylnicotinylalanine analyzed under identical conditions.

\subsection{Experimental}

\section{Isolation of Metabolite}

Female $\mathrm{CD}_{2} \mathrm{~F}_{1}$ mice were dosed with $400 \mathrm{mg} / \mathrm{kg}$ NAL intraperitoneally using a $20 \mathrm{mg} / \mathrm{ml}$ solution of NAL in saline adjusted to $\mathrm{pH}$ 7.4. Urine samples were collected from the treated and control mice for 24 hours and stored at $-80^{\circ} \mathrm{C}$. To prepare samples for analysis, $100 \mu \mathrm{l}$ of methanol were added to $100 \mu \mathrm{l}$ of urine in an Eppendorf ${ }^{\circledR}$ centrifuge tube. After vortex mixing for 2 minutes, the sample was centrifuged and the supernatant was aspirated into a clean tube. The resulting solution was stored at $4^{\circ} \mathrm{C}$ until analysis.

\section{Synthesis of $\mathbf{N}$-acetylnicotinylalanine}

The synthesis of $N$-acetylNAL was accomplished by first weighing $10 \mathrm{mg}$ of standard NAL into a $50 \mathrm{ml}$ round bottom reaction flask. Next, $5 \mathrm{ml}$ of acetic anhydride was added and the resulting solution was stirred overnight at room temperature. The volatile components were removed under vacuum. 


\section{HPLC Conditions}

The liquid chromatography (LC) system consisted of an LC pump (L-7100, Hitachi, Tokyo, Japan), a manual injector (Rheodyne, Rohnert Park, CA) with a $20 \mu 1$ sample loop, and a $2.1 \mathrm{~mm}$ x $150 \mathrm{~mm}(5 \mu$ particle size) reversed-phase liquid chromatography column (SB-C18, HewlettPackard, Avondale, PA). The mobile phase consisted of acetonitrile:1\% acetic acid (20:80) at a flow rate of $100 \mu \mathrm{l} / \mathrm{min}$. The HPLC effluent was delivered to the electrospray ionization source through a $0.100 \mathrm{~mm}$ ID fused silica capillary (Polymicro Technologies, Phoenix, Arizona).

\section{Mass Spectrometry}

Mass spectrometric experiments were performed on ion-trap mass spectrometer a (LCQ, ThermoQuest, San Jose, CA) equipped with an electrospray ion source. Direct infusion experiments were carried out using the syringe pump integral to the LCQ. The positive ion mode was used with a spray voltage of $4 \mathrm{kV}$ applied to the capillary tip. Nitrogen was used as sheath gas at a flow rate of 40 units/min. The heated capillary was maintained at $200^{\circ} \mathrm{C}$.

For LC/MS experiments, the positive ion mode was used with spray voltage of $4.2 \mathrm{kV}$ applied to the electrospray needle. The heated capillary maintained at $200^{\circ} \mathrm{C}$. Nitrogen was employed as both sheath and auxiliary gas at flow rates of 60 and 20 arbitrary units/minute, respectively. The tube lens voltage was set at $0 \mathrm{~V}$, and the octapole ion guides were tuned to maximize ion transmission. 


\subsection{Results and Discussion}

To determine the observed masses of NAL and $N$-acetyl NAL and to optimize the mass spectrometric conditions, standards in water were directly infused into the ESI source at a flow rate of $5 \mu \mathrm{l} / \mathrm{min}$. Data were acquired by scanning from 50 - 500 amu for an acquisition time of one minute. Approximately 150 scans were averaged to generate a mass spectrum for each standard. NAL and synthesized $N$-acetyl-NAL exhibited a base peak corresponding to their quasi-molecular ion, having $\mathrm{m} / \mathrm{z}$ of 195 and 237 respectively. A mobile phase consisting of acetonitrile : $1 \%$ acetic acid in water $(20: 80)$ at a flow rate of $100 \mu 1 / \mathrm{min}$ resulted in baseline resolution between NAL and $N$-acetylNAL with a run time of 5 minutes. Under these conditions NAL eluted with a retention time of 3.1 minutes $(m / z=195)$ [Figure 7.3.1] and the synthetic $N$ acetyl- NAL eluted with a retention time of 3.7 minutes $(\mathrm{m} / \mathrm{z}=237)$ [Figure 7.3.2].

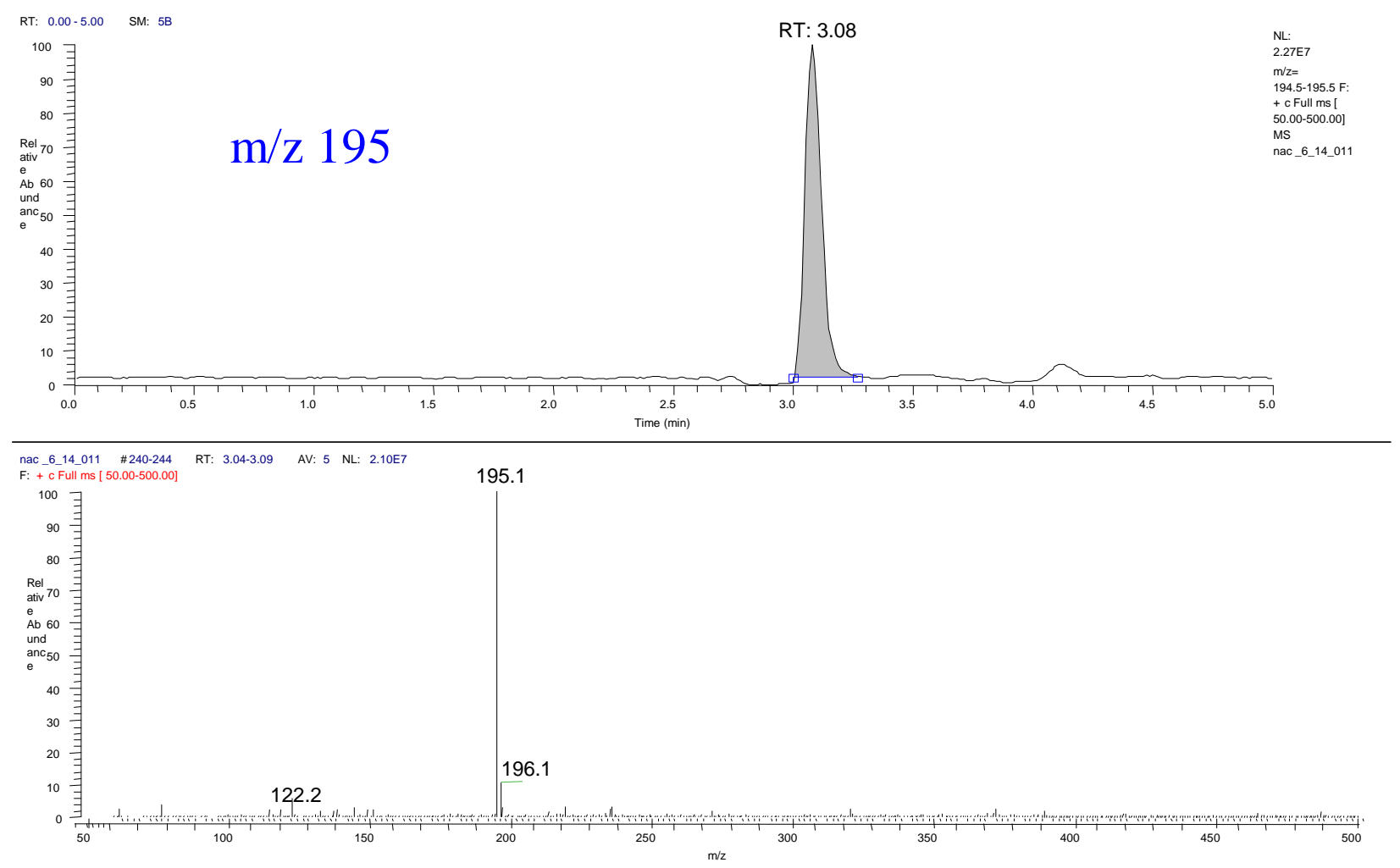

Figure 7.3.1: Selected Ion Chromatogram of m/z 195 and Mass Spectrum of Nicotinylalanine (NAL). 

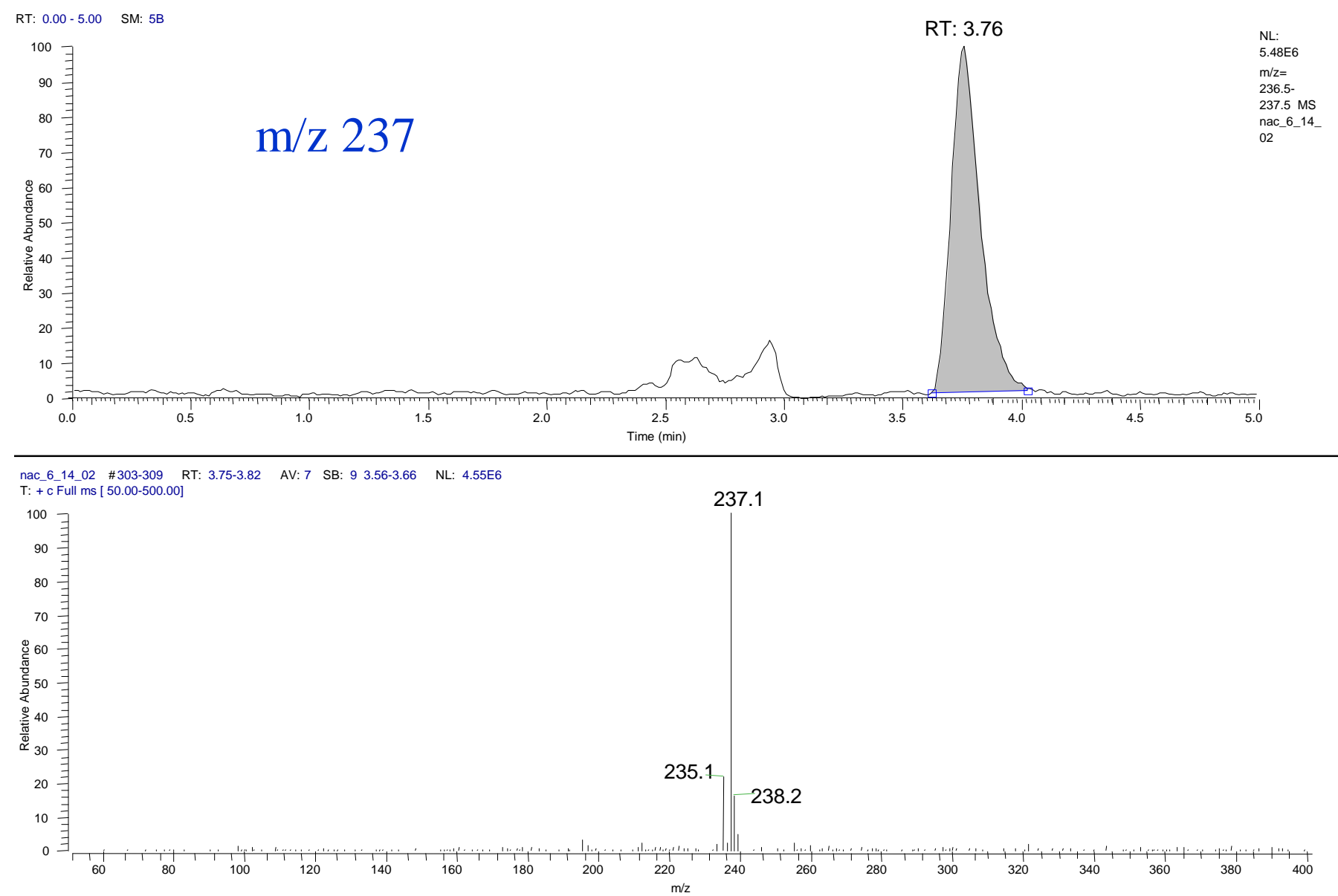

Figure 7.3.2: Selected Ion Chromatogram of $\mathrm{m} / \mathrm{z} 237$ and Mass Spectrum of Synthetic $\mathrm{N}$-acetylnicotinylalanine (N-acetyl NAL)

LC/MS analysis of the mouse urine extract resulted in the observation of a peak with an identical retention time as that of authentic $N$-acetyl NAL. Mass analysis of this component eluting at 3.6 minutes revealed a base peak at $\mathrm{m} / \mathrm{z} 237$ [Figure.7.3.3]. The retention time and the $\mathrm{m} / \mathrm{z}$ observed suggest that the peak in question corresponds to an acetylated metabolite of NAL. 


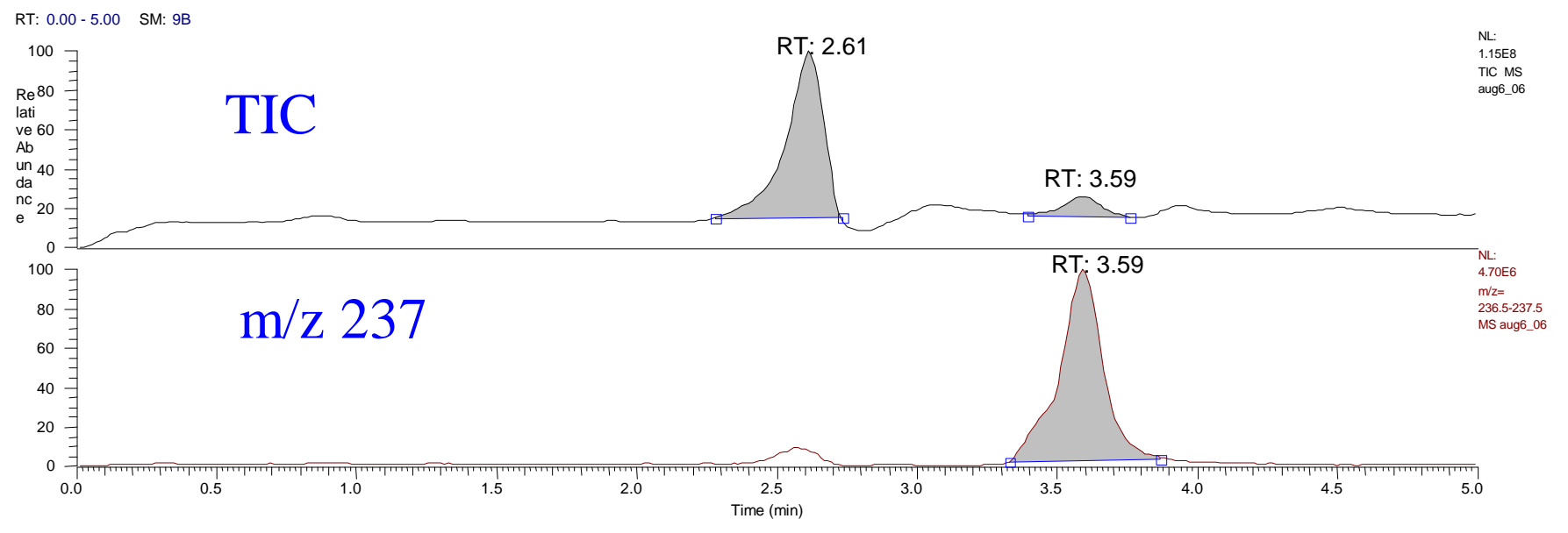

aug6_06 \#255-259 RT: 3.57-3.62 AV: 5 SB: 12 3.25-3.40 NL: 5.37E6

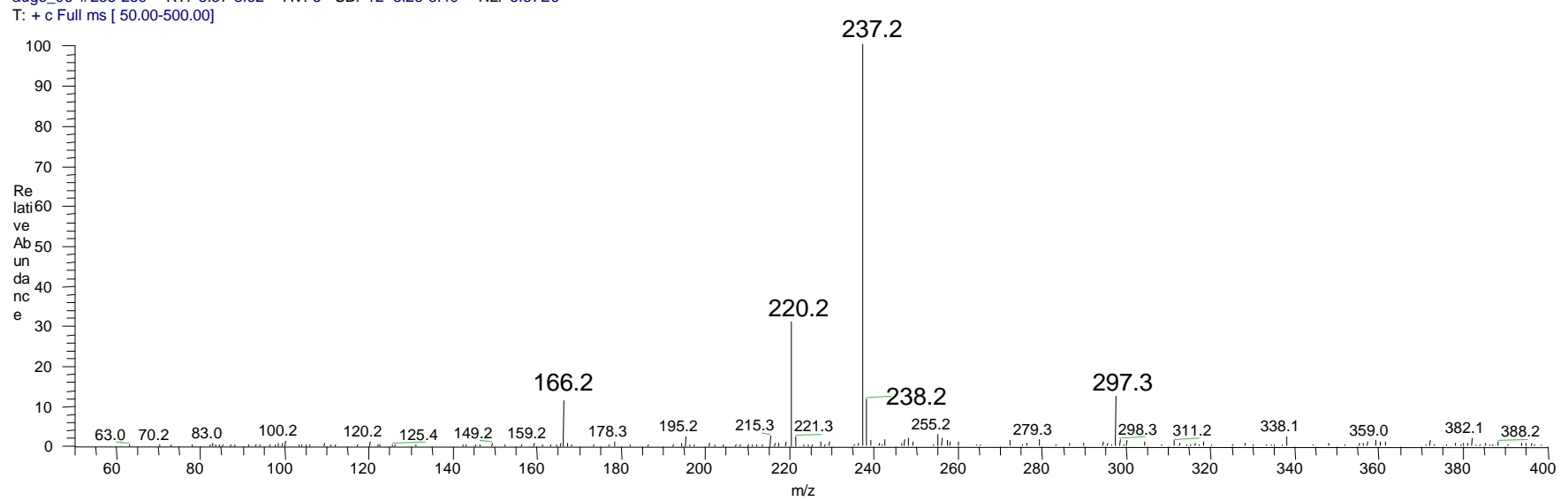

Figure 7.3.3: Chromatogram and Mass Spectrum of the Urine Sample Extract

In order to obtain further information regarding the identity of the metabolite in the mouse urine, a more selective series of tandem mass spectrometric experiments were performed.

The instrument was programmed to trap ions of $m / z, 195$ and 237, then to perform collisioninduced dissociation (CID) of these ions via interaction with helium within the ion trap. First, fragment spectra were collected for the NAL and $N$-acetyl-NAL standards. 
MS/MS of standard NAL $(m / z, 195)$ gave fragment ions at $m / z, 122,149$, and 178 in order of descending intensity. Figure 7.3.4 shows the CID spectrum of standard NAL.

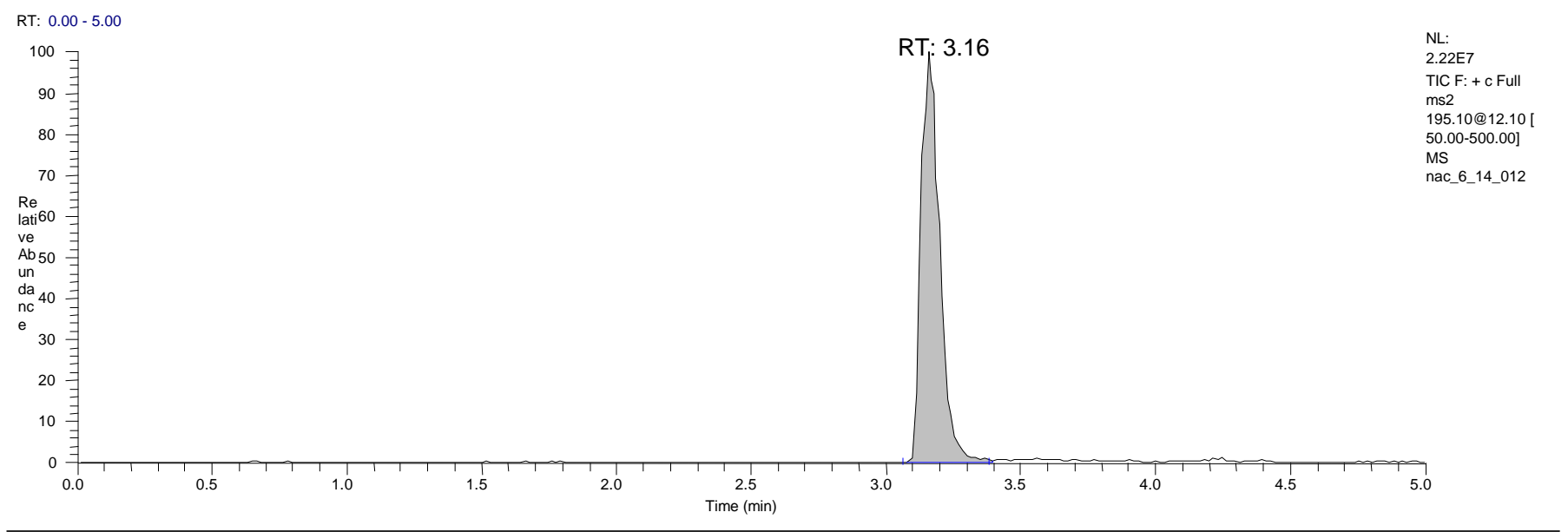

nac_6_14_012 \#193-196 RT: 3.14-3.17 AV: 4 NL: 1.23E7

$\mathrm{F}:$ + c Full ms2 195.10@12.10 [ 50.00-500.00] 122.1

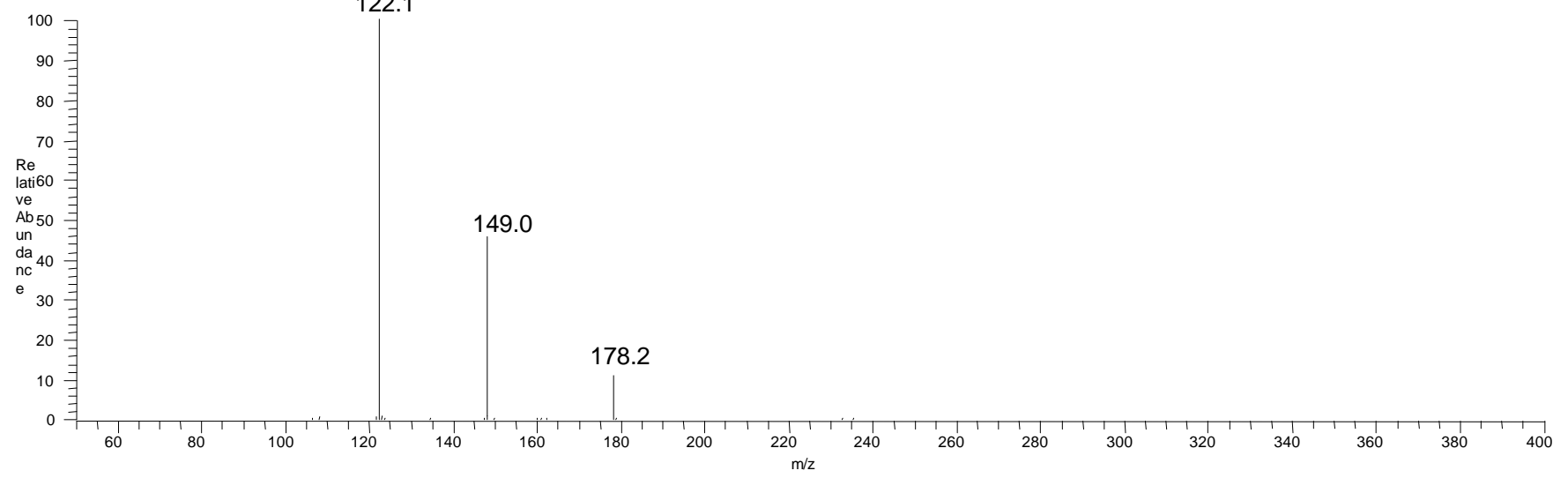

Figure 7.3.4: MS/MS Spectrum of Nicotinylalanine 
MS/MS of standard $N$-acetylNAL $(\mathrm{m} / z, 237)$ gave fragment ions at $\mathrm{m} / z, 178,219$, and 191 in order of descending intensity. Figure 7.3.5 shows the CID spectrum of standard $N$ acetylNAL.

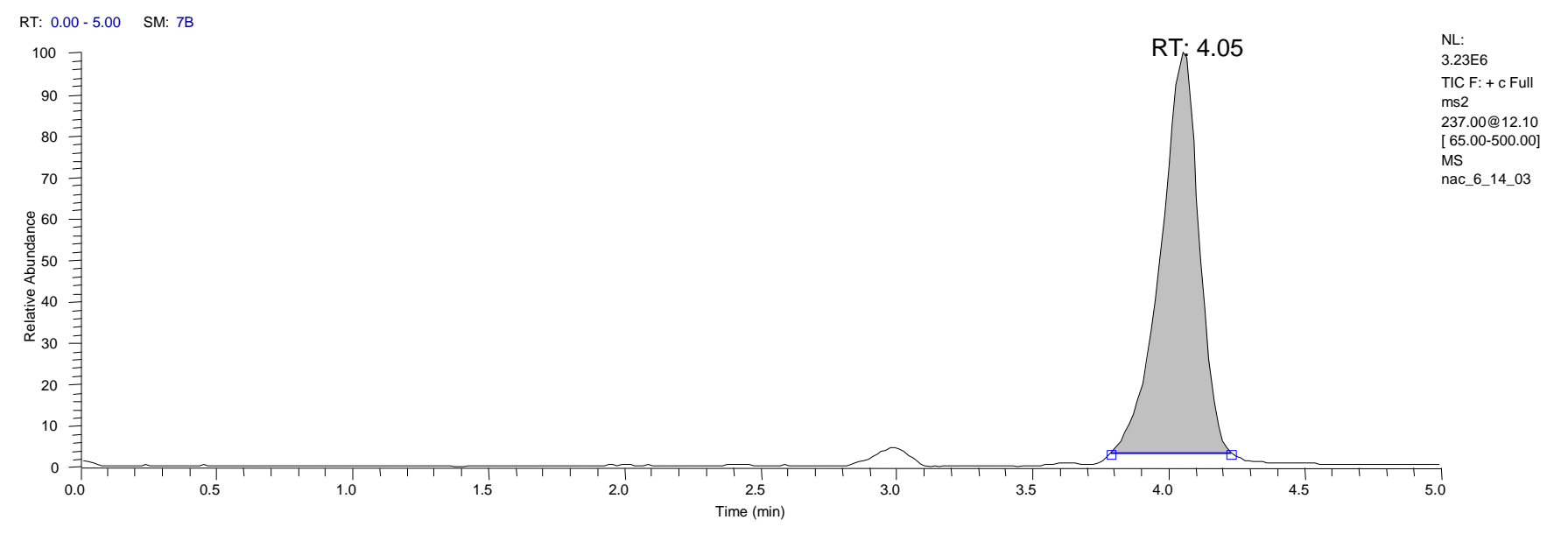

nac_6_14_03 \#240-257 RT: 3.89-4.13 AV: $18 \quad$ NL: 7.90E5

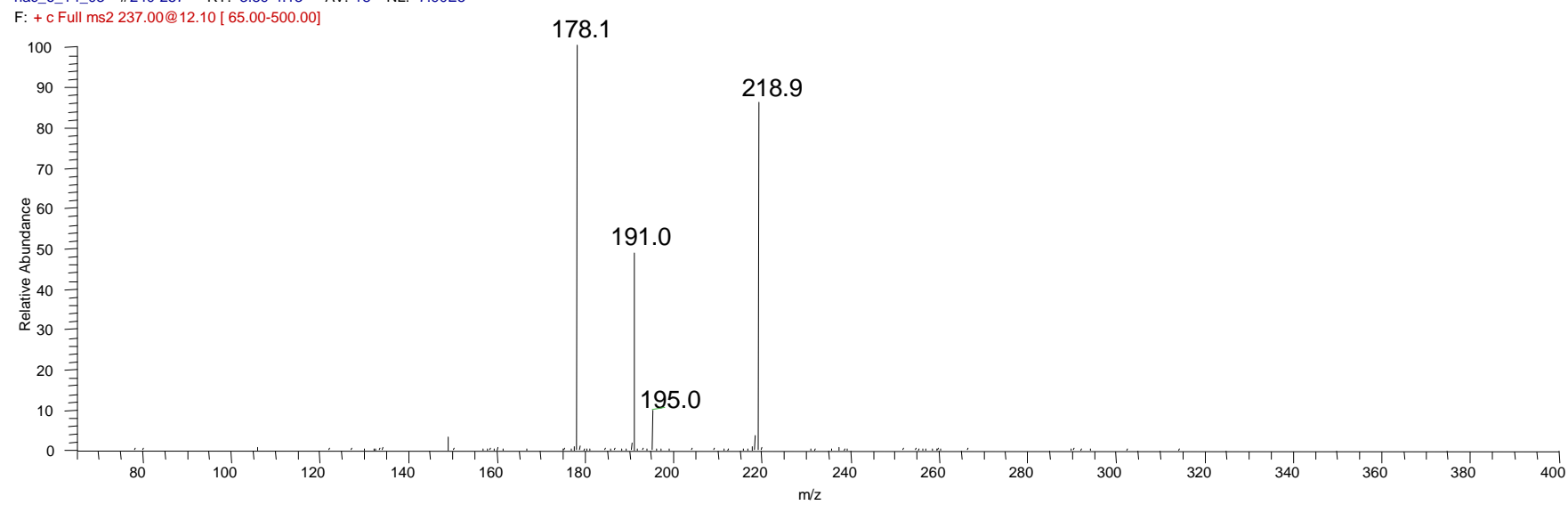

Figure 7.3.5: MS/MS spectrum of Synthetic N-acetyl NAL

Next, CID experiments were performed on the mouse urine extract for chromatographic response for the metabolite peak at 4 minutes $(\mathrm{m} / \mathrm{z} 237)$. The CID spectrum of this peak is 
characterized by fragment ions at $m / z$ 178, 219, and 191 [Figure.7.3.6]. The similarity in the fragment ion spectra of the $N$-acetyl-NAL standard and the peak at 4 minutes observed in the mouse urine extract supports the initial assignment of $N$-acetyl-NAL to the metabolite.

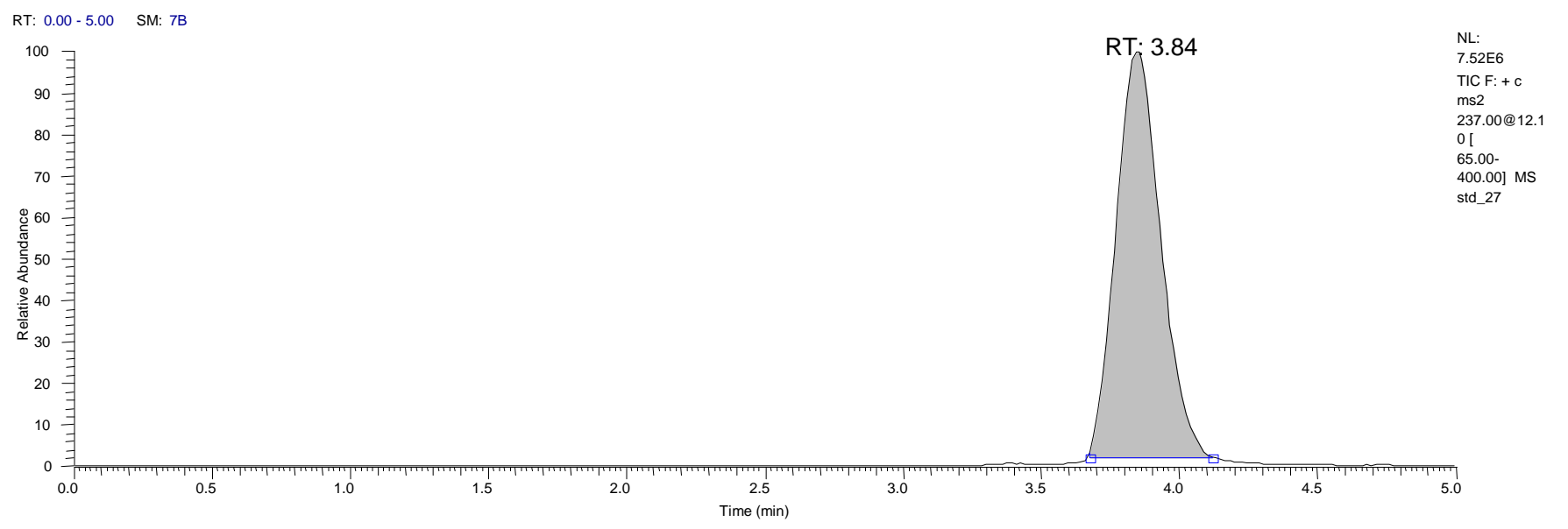

\begin{tabular}{lcrr}
\hline std_27 \#251-262 & RT: $3.85-3.98$ & AV: 12 & NL: $2.02 E 6$
\end{tabular}

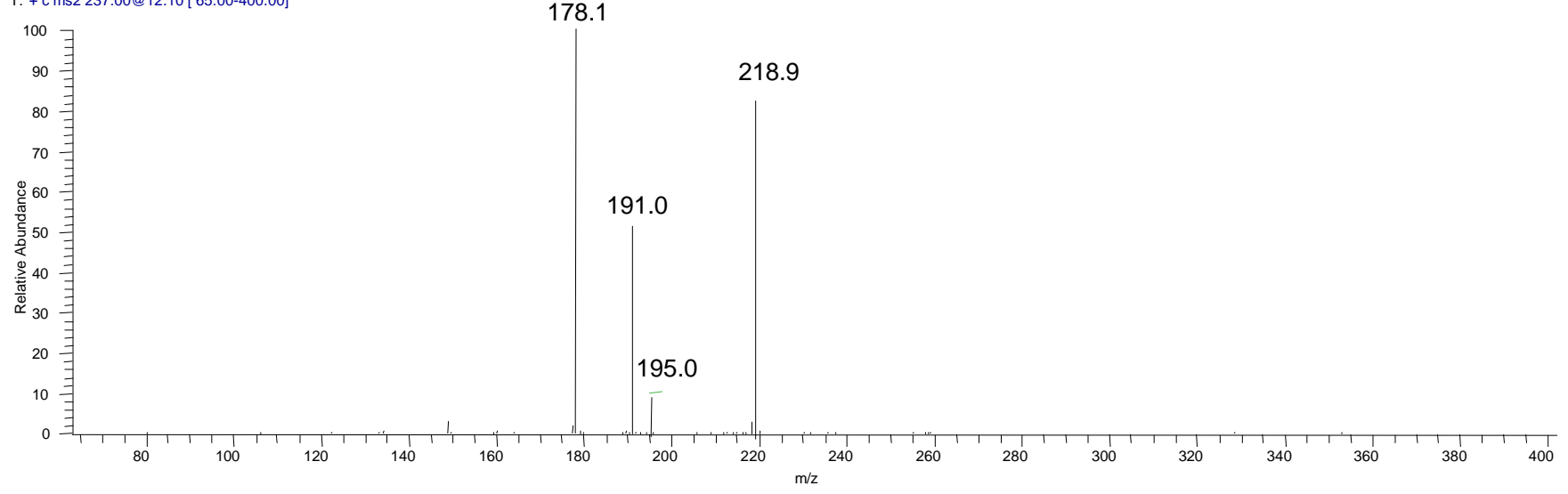

Figure 7.3.6: MS/MS of Urine Sample Extract 
Standard NAL dissociated to give an ion with $\mathrm{m} / \mathrm{z}$ of 178 as one of the fragments; this ion corresponds to the loss of ammonia from the parent molecule. Analogously, standard $N$ acetylNAL dissociated to give a fragment ion with $\mathrm{m} / z .178$ that corresponds to a loss of acetamide.

An additional stage of CID on the fragment with $\mathrm{m} / \mathrm{z} 178$, resulted in ions with $\mathrm{m} / \mathrm{z} 160$, 150, and 132 for standard $N$-acetylNAL [Figure.7.3.7].

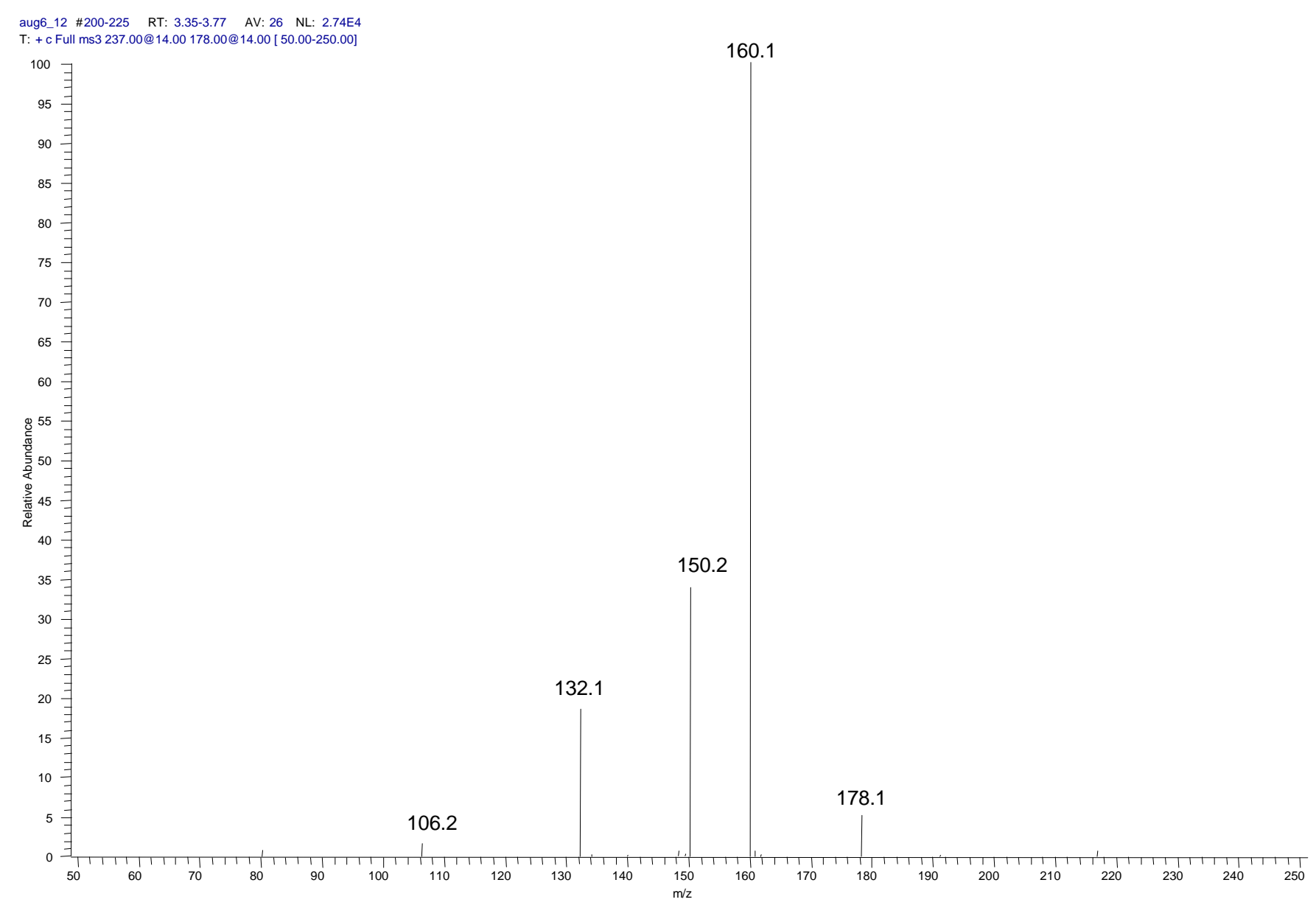

Figure 7.3.7: MS3 of 237, 178 for Synthetic N-acetyl NAL 
Analysis of the mouse urine extract using this $\mathrm{MS}^{3}$ technique yielded identical fragments with $m / z, 160,150$, and 132 for the metabolite [Figure.7.3.8]. The similarity in the $\mathrm{MS}^{3}$ fragmentation of standard $N$-acetylNAL and the metabolite provides further confirmation of the identity of this urinary metabolite.

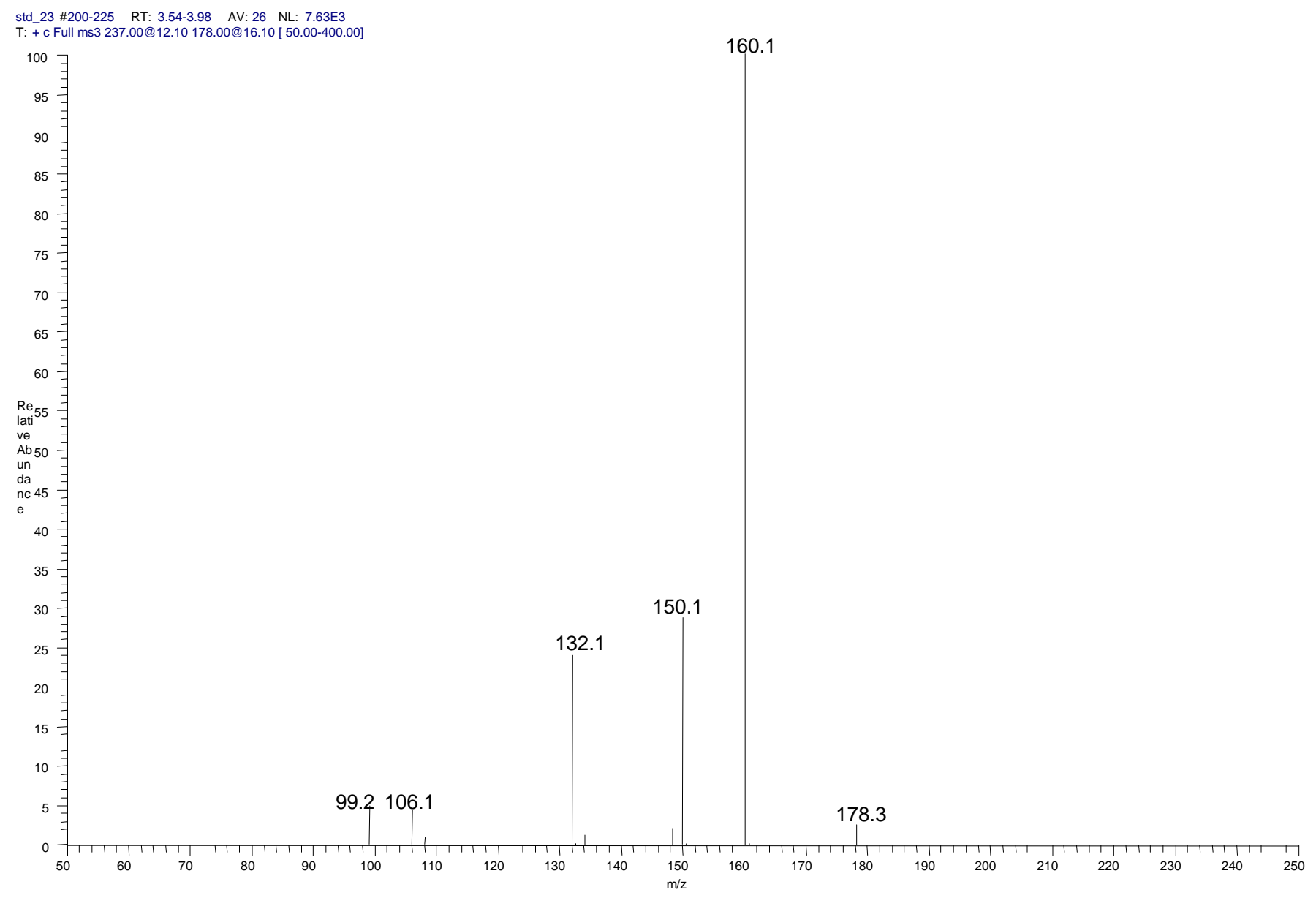

Figure 7.3.8: MS3 of 237, 178 for Urine Sample Extract 
Figure 7.3.9 summarizes the observed fragment ion masses and relative abundances for standard $N$-acetylNAL and the urinary metabolite. Proposed structures for fragments of NAL and $N$-acetylNAL are presented in Fig.7.3.10 and Fig.7.3.11 respectively.

\begin{tabular}{l|lll} 
SAMPLE & $\mathbf{m} / \mathbf{z}$ of Parent Mass & MS2 of $\mathbf{~ m / z ~ 2 3 7 ~}$ & MS3 of 237/178 \\
\hline N-acetyl NAL std & 237 & $178(100)$ & $160(100)$ \\
& & $219(86)$ & $150(35)$ \\
& & $191(49)$ & $132(20)$ \\
& & $195(10)$ & $178(5)$ \\
& & & \\
& & $178(100)$ & $160(100)$ \\
& & $219(83)$ & $150(28)$ \\
& & $191(52)$ & $132(24)$ \\
& & $195(10)$ & $178(3)$
\end{tabular}

Table 7.3.1: Comparison of mass specrometric data for $N$-acetylNAL standard and the Urinary Metabolite. Values in parentheses are \% relative abundance. 


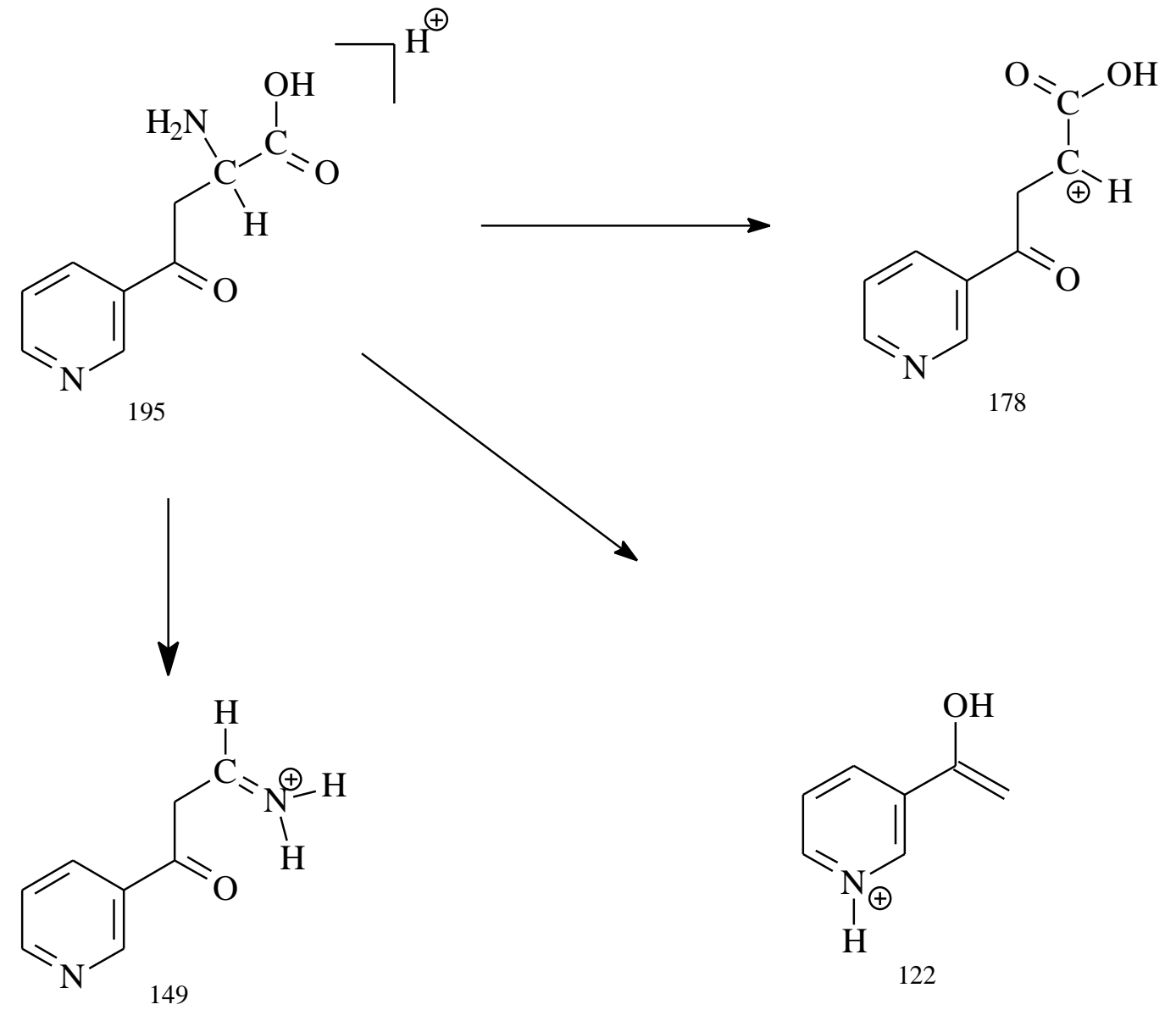

Figure 7.3.9: Proposed structures of daughter ions of NAL 

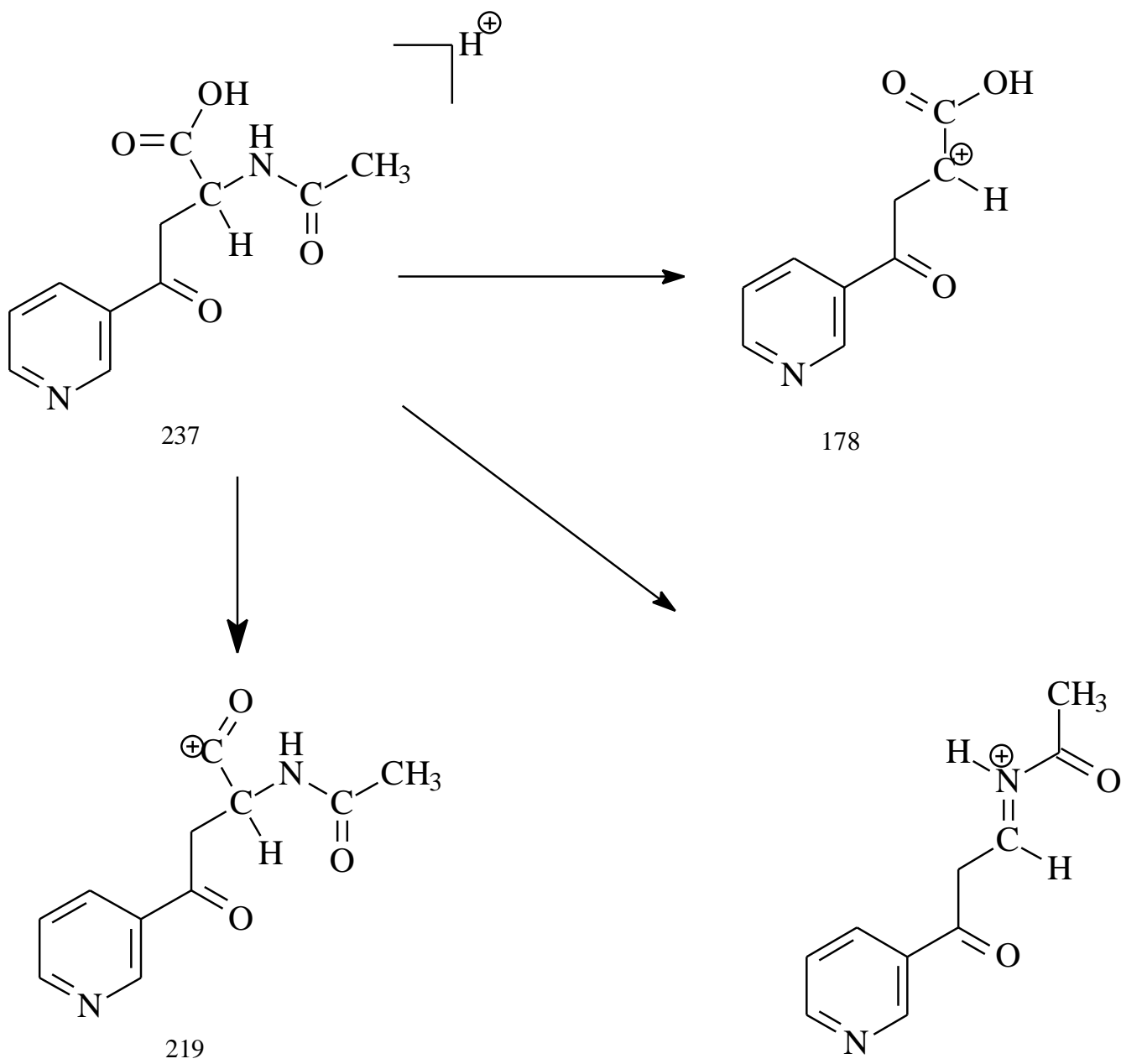

191

Figure 7.3.10: Proposed structures of daughter ions of $N$-acetylNAL 


\subsection{Conclusion}

On the bases of chromatographic retention time, single-stage mass spectrometric analysis, and multiple-stage mass spectrometric analysis, we conclude that the identity of the unknown metabolite is $\mathrm{N}$-acetyl nicotinylalanine. Retention time data along with mass spectral information provide compelling evidence, and the added selectivity of multiple-stage MS experiments further support this assignment. Based upon this conclusion, the proposed metabolism is indicated in Figure 7.4.1.

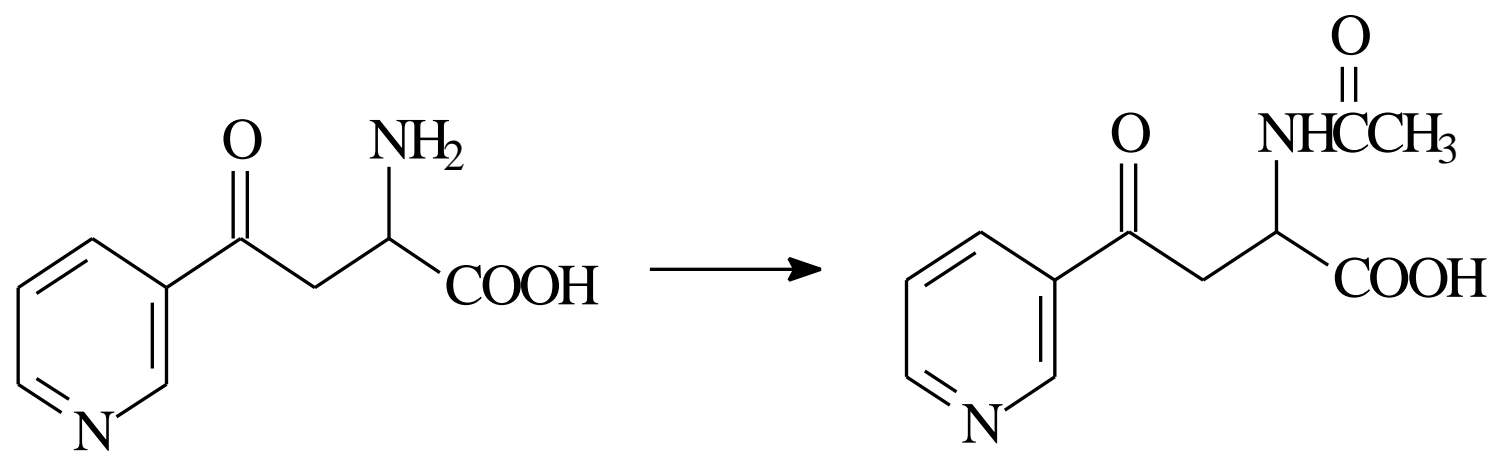

Figure 7.4.1: Proposed Metabolism of NAL 
Curriculum Vita for Gregg A. Pratt 


\section{Gregg A. Pratt}

34 Delrose Drive

Morgantown, WV 26508

Work: (304)293-3435 x4218

Home: (304)296-1166

email: gpratt@wvu.edu

Gregg.Pratt@Quintiles.com

\section{Experience:}

8/00-present Senior Associate Scientist/Group Leader - Biopharmaceutics

Quintiles, Inc., Kansas City, MO

LC/MS-based bioanalytical methods development and method troubleshooting. Analytical project management assuring GLP compliance. Personnel management including training, scheduling, and performance evaluation.

\section{3/95-5/97 Senior Chemist, Pharmacokinetics}

Mylan Pharmaceuticals, Inc., Morgantown, WV

Developed quantitative analytical methods for the analysis of pharmaceutical compounds and metabolites in biological matrices using GC/MS and LC/MS instrumentation. Conducted method validation assuring GLP compliance. Managed clinical studies, including supply procurement and personnel scheduling and management. Prepared analytical reports for FDA submissions.

Operated and maintained MS instrumentation.

\section{6/89-3/95 Chemist, Pharmacokinetics}

Mylan Pharmaceuticals, Inc., Morgantown, WV

Prepared samples for pharmacokinetic studies. Operated and maintained Finnigan TSQ mass spectrometers. Developed and assisted in the development of analytical methods. Managed junior personnel. Set up and maintained LC, GC, and MS instrumentation for pharmacokinetic applications. Maintained laboratory SOP manual. Maintained general laboratory equipment.

\section{Education:}

$9 / 2000$

\section{Doctorate}

West Virginia University, Morgantown, WV

*Ph.D. in Analytical Chemistry

*research interests: protein analysis using ion-trap mass spectrometry, and drug metabolite characterization by LC/ESI/MS/MS

5/1989 Bachelors Degree

The University of Wyoming, Laramie, WY

* BS in Biology with a Chemistry minior

6/1979 High School

Plum Senior High School, Pittsburgh, PA

* graduation with honors

\section{Affiliations:}

Phi Lambda Upsison Chemistry Honorary - member

American Chemical Society - member

American Society for Mass Spectrometry - member

Society for Molecular Imprinting - member 


\section{Gregg A. Pratt}

Skills:

Skill name

Analytical method development

Finnigan TSQ mass spectrometer

Finnigan LCQ mass spectrometer

Hewlett-Packard and Varian

Gas Chromatographs

Waters LC equipment

Analytical Project Management

Personnel Management level

expert

expert

expert

expert

expert

expert

expert experience

8 years

8 years

3 years

10 years

11 years

5 years

5 years

\section{Training:}

"Chemical Separations", Waters Assoc., completed August 1989

"Finnigan TSQ Operation and Maintenance"- completed January 1991

"Advanced Techniques in Capillary Gas Chromatography" - Varian Associates, completed May 1993

\section{Presentations and Publications:}

Girish S. Gudi, Patrick S. Callery, Gregg A. Pratt, and Fred L. King, "Identification of a Urinary Metabolite of Nicotinylalanine by LC/ESI/MS and Hydrogen-Deuterium Exchange", manuscript in preparation. Data also presented at WVU School of Medicine Research Day, Morgantown, WV and $32^{\text {nd }}$ Annual Graduate Student Symposium in Medicinal Chemistry, Buffalo, NY.

Gregg A. Pratt and Fred L. King, "Energetics of Heme Unit Dissociation from Heme Proteins", manuscript in preparation. Data presented at the $48^{\text {th }}$ Conference of The American Society for Mass Spectrometry, Long Beach, CA, June 11-15, 2000.

Gregg A. Pratt and Fred L. King, "Influence of Sheath Liquid Composition on the ESI/MS Charge-State Distribution of Heme Proteins", manuscript in preparation. 


\section{References}

${ }^{1}$ Thompson, JJ; R ays of Positive Electricity, Longman's Green: London, 1930

2 Munson, B; Field, F; J A m Chem Soc, 1966, 88, 2621.

3 Blakeley, CR; Vestal, MI; A nal Chem, 1983, 51, 750-754.

4 Willoughby, RC; Browner, RF; A nal Chem, 1984, 56, 2626-31.

${ }^{5}$ Fenn, JB; Mann, M; Meng, CK; Wong, SF; Whitehouse, CM; Science, 1989, 246, 64-71.

6 Bakhtiar, R; Hofstadler, SA; Smith, RD; J Chem Ed, 1996, 73, A118-A 123.

7 Ii, T, et.al.; Carbohydrate Research, 1995, $273,27$.

8 McLuckey, SA; Van Berkel, GJ; Glish, GL; J A m Soc M ass Spectrom, 1992, 3, 60-70.

${ }^{9}$ Loo, JA; Udseth, HR; Smith, RD; Rapid Commun M ass Spectrom, 1988, 2, 207-210.

${ }_{10}$ Chowdhurt, SK; Katta, V; Chait, BT; J A m Chem Soc, 1990, 112, 9012-9013.

11 M cLuckey, SA; Stephenson, JL; A sano, KG; A nal Chem, 1998, 70, 1198-1202.

12 Li, YT; Hsieh, YL; Henion, JD; Ganem, B, J A m Soc M ass Spectrom, 1993, 4, 631-637.

13 Zeleny, J; J Phys Rev, 1917, 10, 1.

${ }^{14}$ Dole, M; Mack, LL; Hines, RL; Mobley, RC; Ferguson, LD; Alice, MB; J Chem Phys, 1968, 49, 2240.

${ }^{15}$ Covey, TR; Bonner, RF; Shushman, BI; Henion, J; Rapid Comm M ass Spectrom, 1988, 2, 249-256.

16 Iribarne, JV; Thomson, BA; J Chem Phys, 1976, 64, 2287.

${ }_{17}$ Rollgen, FW; Bramer-Wegner, E; Buttering, L; J Phys Colloq, 1984, 45, Supplement 12, C9-297.

18 Van Berkel, GJ; Glish, GL; McLuckey, SA; A nal Chem, 1990, 63, 1284-1295.

19 Paul, W; Steinwedel, ZZ; N aturforsh, 1953, 8a, 448-450.

20 Paul, W; Steinwedel, ZZ; N aturforsh A, 1956, 104, 672.

21 Paul, W; Steinwedel, Z, German Patent 944900, 1956.

22 Stafford, GC; Kelley, P; Stephens, DR; US Patent 4540884, 1985

${ }^{23}$ Stafford, GC; Kelley, PE; Syka, JEP; Reynolds, WE; Todd, JFJ; Int J M ass Spectrom Ion Proc, 1984, 60, 85-98.

${ }^{24}$ Cole, RB; Electrospray Ionization M ass Spectrometry: Fundamentals, Instrumentation and A pplications, John Wiley\& Sons: New York, 1997.

${ }_{25}$ Yost, RA; Enke, CG; A nal Chem, 1979, 51, 1251A-1264A.

${ }^{26}$ Voet, G; Voet, JG; Biochemistry, John Wiley \& Sons: New York, 1990.

27 Branden, C; Tooze, J; Introduction to Protein Structure, $2^{\text {nd }}$ Edition, Garland: New York, 1999.

28 Stryer, L; Biochemistry, 3rd Edition, WH Freeman: New York, 1988.

29 Zubay, G, Biochemistry, Addison-Wesley: New York, 1983, pp 121-125.

30 Figures from http/ / www.chem.uci.edu/ -pfarmer, research homepage of Professor

Paul Farmer, Department of Chemistry, University of California at Irvine.

31 Evans, SV; Brayer, GD; J M ol Bio, 1990, 213, 885-897.

32 Fei, H; Hendrickson, CL; Marshall, AG; J A m Soc M ass Spec; 2000, 11, 120-126.

33 M cFarlene, RD; Torgerson, DF; Science, 1976, 191, 920. 
34 Barber, M; Bordoli, RS; Sedgwick, RD; Tyler, AN; N ature, 1981, 293,270.

35 Loo, JA; Ogorzalek-Loo, RR; Udseth, HR; Smith, RD; Rapid Commun M ass Spectrom, 1991, 5, 101-105.

36 Konermann, L; Rosell, FI; Mauk, AG; Douglas, DJ; Biochemistry, 1997, 36, 6448-6454.

37 Sogbein, OO; Simmons, DA; Konermann, L; J A m Soc M ass Spectrom, 2000, 11, 312-319.

38 Konermann, L; Collings, DA; Douglas, DJ; Biochemistry, 1997, 36, 5554-5559.

39 Thivenon-Emeric, G; Kozlowski, J; Zhang, Z; Smith, DL; A nal Chem, 1992, 64, 2456-

2458.

40 Resing, KA; Hoofnagle, AN; Ahn, NG; J A m Soc M ass Spectrom, 1999, 10, 685-702..

41 Eyles, S]; Speir, JP; Kruppa, GH; Gierasch, LM; Kaltashov, IA; J A m Chem Soc, 2000, $122,495-500$.

42 Fenn, ; et.al.; J A m Soc M ass Spectrom, 1993, 4, 524-535.

43 Loo, JA; et.al., M ass Spectrom Rev, 1997, 16, 1-23.

44 Busman, M; et. al., Rapid Commun M ass Spectrom, 1994, 8, 211-216.

45 Ashton, DS; et.al., Biochem Biophys Res Comm, 1994, 199, 2, 694-8.

46 Kebarle, $\mathrm{P}$; Tang, $\mathrm{H}$; in Electrospray Ionization M ass Spectrometry: Fundamentals, Instrumentation and A pplications, Chapter 1 John Wiley\& Sons: New York, 1997

${ }^{47}$ Caflish, A; Karpus, $M$; in The Protein Folding Problem and Tertiary Structure Prediction, Ch 7, Birkhauer: Boston, 1994.

48 M Llafferty, FW; Guan, Z; Haupts, U; Wood, JD; Kelleher, NL, J A m Chem Soc, 1998, 120, 19, 4732-40.

49 Wang, F; Freitas, MA; Marshall, AG; Sykes, BD; Int J M ass Spectrom, 1999, 192, 319-

325.

50 M irza, UA; Chait, BT; Int J M ass Spectrom, 1997, 162, 173-181.

51 Hunter, CL; Mauk, AG; Douglas, DJ; Biochemistry, 1997, 36, 1018-1025

52 Evans, SV; Brayer, GD; J M olec Biol, 1990,213, 885-897.

53 Katta, V; Chait, BT; I A m Chem Soc, 1993, 115, 6317-6321.

54 Wang, F; Tang, X; Biochemistry, 1996, 35, 4069-4078.

55 Eyles, SI; Radford, SE; Robinson, CV; Dobson, CM ; Biochemistry, 1994, 33, 1303813048.

56 Moroni F, Russi P, Gallo-Mezo MA, Moneti G, Pellicciari R; J N eurochem 1991, 57, 1630-1635.

57 Murray MF, Nghiem M, Srinivasan A, Biochem Biophys Res Commun 1995;212:126-131.

${ }^{58}$ Child SJ, Franke CA, Hruby DE. Virus Res 1988:9:119-132.

59 Eiseman, JL, Ramlsand, RS, Sent, DL, and Shaskan, EG. Evaluation of the Pharmacokinetics \& Efficacy of Racemic Nicotinylalanine Alone and in Combination with A cetylsalicylic A cid in a Murine Model of AIDS, MAIDS 2000. 